Classificação de singularidades simpléticas:

o método das restrições algébricas 



\title{
Classificação de singularidades simpléticas: o método das restrições algébricas
}

\section{Lito Edinson Bocanegra Rodríguez}

\author{
Orientadora: Profa. Dra. Roberta Godoi Wik Atique
}

Dissertação apresentada ao Instituto de Ciências Matemáticas e de Computação - ICMC-USP, como parte dos requisitos para obtenção do título de Mestre em Ciências - Matemática . VERSÃO REVISADA 
Ficha catalográfica elaborada pela Biblioteca Prof. Achille Bassi e Seção Técnica de Informática, ICMC/USP, com os dados fornecidos pelo(a) autor(a)

Bocanegra Rodríguez, Lito Edinson
Classificaça de singularidades simpléticas: o
método das restriçóes algébricas / Lito Edinson
Bocanegra Rodríguez; orientadora Roberta Godoi Wik
Atique. -- São Carlos, 2014 .
84 p.
Dissertação (Mestrado - Programa de Pós-Graduação
em Matemática) -- Instituto de Ciências Matemáticas
e de Computação, Universidade de São Paulo, 2014.
1. Restrições algébricas. 2. Classificação de
curvas numa variedade simplética. 3. Ponômios quase-
homogêneos. I. Wik Atique, Roberta Godoi, orient.
II. Título.


A minha família, que sempre me apoiou, especialmente minha mãe Bernardina Rodríguez e meu pai Tomás Bocanegra. 



\section{Agradecimentos}

Primeiramente quero agradeçer em especial a minha orientadora Profa. Dra. Roberta Godoi Wik Atique, pela sua dedicação, paciência, ajuda, amizade, apoio em todo o tempo que ela me orientou.

Aos meus queridos pais, Bernardina Rodríguez e Tomás Bocanegra que sempre me apoiaram, me deram seu compreensão e amor e pelo sacrifício que fizeram para continuar com meus estudos e chegar até onde estou. Às minhas irmãs Vanesa e kelbi assim como a minha tia Guadalupe Bocanegra pela amizade e pelo apoio de forma incondicional durante toda minha vida. A Edith Cuenca, pelo amor e comprensão incondicional que sempre foram necesários para continuar.

Aos professores do departamento de Matemática do ICMC que contribuiram com a minha formação: Eduardo Tengan, Carlos Grossi, Nivaldo de Góes, Carlos Maquera, Ederson Moreira, Daniel Levcovitz, Paulo Dattori, Leandro Aurichi, Sérgio Monari e Farid Tari pelos ensinamentos, apoio e motivação.

Aos funcionários da Biblioteca do ICMC, das seções de pós-graduação, da área financeira e da diretoria.

Aos meus amigos Patricia Hilario, Norbil Cordova, Elizabeth Salazar, Edith Anco, Ginnara Mexia, Patricia Tempesta, Apoeña Passos, Thales Maier, Leandro Nery, Camila Ruiz, ... todos eles tem minha gratidão por todo o apoio.

À CAPES pelo apoio financeiro sim o qual não teria sido possível este trabalho.

Peço desculpas às pessoas que não citei e que me ajudaram direta ou indireitamente no mestrado, agradeço muito. 


\section{Resumo}

Neste trabalho obtemos a classificação simplética de singularidades de curvas do tipo A, D, E, através do método das restriçães algébricas.

Em outras palavras, considerando $\left(\mathbb{R}^{2 n}, \omega\right)$ um germe de variedade simplética, $N=$ $\left\{H\left(x_{1}, x_{2}\right)=x_{\geqslant 3}=0\right\}$ uma curva algébrica singular onde $H\left(x_{1}, x_{2}\right)$ é uma função que representa uma das clássicas singularidades $A_{k}, D_{k}, E_{6}, E_{7}, E_{8}$, queremos classificar com respeito ao grupo dos simplectomorfismos as classes das variedades algébricas em $\left(\mathbb{R}^{2 n}, \omega\right)$ que são difeomorfas a $N$, através do método das restrições algébricas. 


\section{Abstract}

In this work, we obtain the classification of symplectic singularities of curves of the type A, D, E, through the algebraic restriction method.

In other words, considering $\left(\mathbb{R}^{2 n}, \omega\right)$ a germ of symplectic manifold, $N=\left\{H\left(x_{1}, x_{2}\right)=\right.$ $\left.x_{\geqslant 3}=0\right\} \subset\left(\mathbb{R}^{2 n}, \omega\right)$ an algebraic singular curve where $H\left(x_{1}, x_{2}\right)$ is a function that represents one of the classical singularities $A_{k}, D_{k}, E_{6}, E_{7}, E_{8}$, we want to classify with respect to the group of symplectomorphisms the classes of varieties in $\left(\mathbb{R}^{2 n}, \omega\right)$ that are diffeomorphic to $N$, through the algebraic restrictions method. 


\section{Sumário}

Notação $\quad$ ix

Introdução

1 Preliminares 3

1.1 Variedades Simpléticas . . . . . . . . . . . . . . . . . 3

1.1.1 Campos vetoriais . . . . . . . . . . . . . . 4

1.1.2 Formas diferenciáveis . . . . . . . . . . . . . 5

1.1.3 Derivada de Lie . . . . . . . . . . . . . . . 7

1.1.4 Variedades Simpléticas . . . . . . . . . . . . 8

1.2 Teoria de Singularidades . . . . . . . . . . . . . . . . . 9

1.2.1 Germes e Jatos . . . . . . . . . . . . . . . . 9

1.2 .2 Ação de Grupo . . . . . . . . . . . . . . . . . . . 11

1.2 .3 A álgebra $\varepsilon_{n} \ldots \ldots \ldots \ldots \ldots \ldots \ldots$

1.2.4 Germes Simples . . . . . . . . . . . . . . . 14

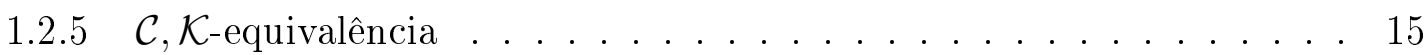

1.3 Variedades Algébricas . . . . . . . . . . . . . . . . 16

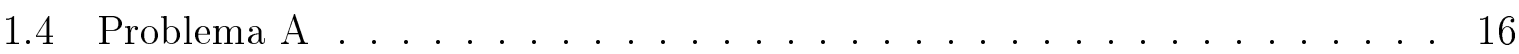

2 O método das restrições algébricas $\quad 19$

2.1 Definição de restrição algébrica . . . . . . . . . . . . . . . 19

2.2 A ação do grupo das simetrias locais . . . . . . . . . . . . . . 22

2.3 Teorema de Redução . . . . . . . . . . . . . . . . . . . . . . 23

2.4 Grupos de Cohomologia Relativa . . . . . . . . . . . . . . 26

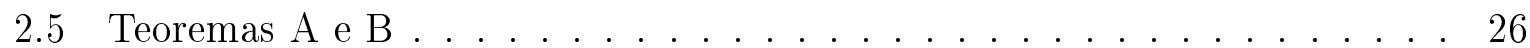


3 Invariantes Simpléticos $\quad 34$

3.1 Teorema C e D . . . . . . . . . . . . . . . . 34

3.2 O espaço $\left[\Lambda^{2 \text {,closed }}\left(\mathbb{R}^{2 n}\right)\right]_{N}$ e o conjunto $\left[\operatorname{Symp}\left(\mathbb{R}^{2 n}\right)\right]_{N} \ldots \ldots \ldots$

4 O método das restrições algébricas para curvas 41

4.1 As simetrias infinitesimais da curva $\{H=0\} \ldots \ldots \ldots$. . . . . . 42

4.2 Restrições algébricas de curvas quase-homogêneas . . . . . . . . . . . . . 47

4.3 Ferramentas para a Classificação . . . . . . . . . . . . . 50

5 Classificação simplética de curvas planas quase-homogêneas singulares 53

5.1 O espaço das restrições algébricas . . . . . . . . . . . . . . 54

5.2 O índice de isotropia e a multiplicidade simplética . . . . . . . . . . 55

5.3 Classificação simplética A-D-E . . . . . . . . . . . . . . . . 58

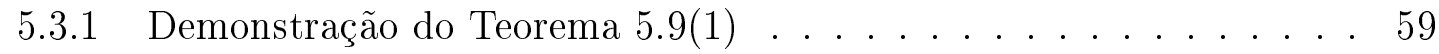

5.3 .2 Demonstração do Teorema $5.9(2) \ldots \ldots$. . . . . . . . 67

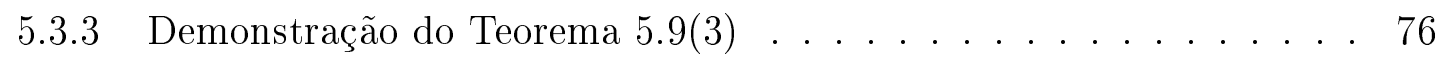

5.3.4 Demonstração do Teorema 5.9(4) . . . . . . . . . . . . 77

Referências Bibliográficas $\quad 83$ 


\section{Notação}

$\mathbb{Z}$

$\mathbb{N}$

$\mathbb{N}_{0}$

$\mathbb{R}$

$\operatorname{dim} M$

$\operatorname{dim}_{\mathbb{R}} Q$

$\mathbb{R}\left\{a_{1}, \ldots, a_{k}\right\}$

$d$

$\wedge$

*

$\mathscr{L}_{V}$

$i_{V}$

$i_{M}$

$(M, 0)$

$\left(\mathbb{R}^{2 n}, \omega\right)$

$\operatorname{Symp}\left(\mathbb{R}^{2 n}\right)$

$\varepsilon_{n}$

$\mathscr{M}_{n}$

$\mu(f)$

$\Lambda^{p}(M)$

$\Lambda^{0}\left(\mathbb{R}^{m}\right)$

$\Lambda_{N}^{p}(M)$

$\mathscr{A}_{0}^{p}(N, M)$

$\left[\Lambda^{p}(M)\right]_{N}$

$[\omega]_{N}$

$\left\langle f_{1}, \ldots, f_{k}\right\rangle$

$\langle\nabla f\rangle$

$\left\langle[\theta]_{N}\right\rangle$

$\left[\Lambda^{2, \text { closed }}\left(\mathbb{R}^{2 n}\right)\right]_{N}$

$\left[\operatorname{Symp}\left(\mathbb{R}^{2 n}\right)\right]_{N}$ conjunto dos números inteiros.

conjunto dos números inteiros positivos.

conjunto dos números inteiros não negativos.

conjunto dos números reais.

dimensão da variedade $\mathrm{M}$.

dimensão do $\mathbb{R}$-espaço vetorial $Q$.

o $\mathbb{R}$-espaço vetorial gerado por $a_{1}, \ldots, a_{k}$.

derivada exterior.

produto exterior de formas diferenciáveis.

pullback.

derivada de Lie, onde $V$ é campo de vetores.

produto interior, onde $V$ é campo de vetores.

função inclusão, onde $M \subset \mathbb{R}^{m}, m \in \mathbb{N}$.

germe do conjunto ou subvariedade $M$ onde $0 \in M \subset \mathbb{R}^{m}, m \in \mathbb{N}$.

germe de variedade simplética na origem.

o conjunto das formas simpléticas em $\mathbb{R}^{2 n}$.

o $\mathbb{R}$-espaço vetorial dos germes na origem de funções em $\mathbb{R}^{n}$.

o ideal máximal de $\varepsilon_{n}$.

número de Milnor de f.

o espaço de todos os germes na origem, das $p$-formas diferenciáveis em $M$.

o $\mathbb{R}$-espaço vetorial dos germes na origem de funções em $\mathbb{R}^{m}$.

$=\left\{\omega \in \Lambda^{p}(M): \omega(x)=0, \forall x \in N\right\}$.

$=\left\{\alpha+d \beta: \alpha \in \Lambda_{N}^{p}(M), \beta \in \Lambda_{N}^{p-1}(M)\right\}$.

o $\mathbb{R}$-espaço vetorial das restriçoes algébricas a $N$ de todas as $p$ formas em $M$.

restrição algébrica da $k$-forma ao conjunto $N$.

o ideal gerado por $f_{1}, \ldots, f_{k} \in \Lambda^{0}\left(\mathbb{R}^{m}\right)$.

ideal gerado pelas derivadas parciais de $f \in \Lambda^{0}\left(\mathbb{R}^{m}\right)$.

o $\Lambda^{0}(M)$-submódulo do $\Lambda^{0}(M)$-módulo $\left[\Lambda^{p}(M)\right]_{N}$

o subespaço de $\left[\Lambda^{2}\left(\mathbb{R}^{2 n}\right)\right]_{N}$ composto das restrições algébricas a $N$ de todas as 2-formas fechadas em $\mathbb{R}^{2 n}$.

o conjunto aberto em $\left[\Lambda^{2 \text {,closed }}\left(\mathbb{R}^{2 n}\right)\right]_{N}$ composto das restriões algébricas a $N$ das 2-formas simpléticas em $\mathbb{R}^{2 n}$. 
TM fibrado tangente da variedade $\mathrm{M}$.

$j^{k} f(0) \quad k$-jato na origem de $f \in \Lambda^{0}\left(\mathbb{R}^{m}\right)$.

$\left.\omega\right|_{T M} \quad$ restrição da $k$-forma diferenciável à subvariedade $M$.

$D_{n} \quad$ grupo do germe de difeomorfismos de $\left(\mathbb{R}^{n}, 0\right)$.

$D(M) \quad$ grupo do germe de difeomorfismos do germe de subvariedade $(M, 0)$.

$T(a) \quad$ o espaço tangente a $a \in\left[\Lambda^{2}\left(\mathbb{R}^{2 n}\right)\right]_{N}$.

$E, \mathscr{H} \quad$ campo vectorial de Euler e Hamiltoniano respectivamente.

Derlog $\{N\}$ conjunto das simetrias infinitesimais da curva $N \subset\left(\mathbb{R}^{2}, 0\right)$.

$a^{(d)} \quad$ parte quase homogênea de grau $d$ de $a \in\left[\Lambda^{2}\left(\mathbb{R}^{2}\right)\right]_{\{H=0\}}$.

$\circ(\delta) \quad$ o subespaço de $\left[\Lambda^{2}\left(\mathbb{R}^{2}\right)\right]_{\{H=0\}}$ das restrições algébricas sem termos quase-homogêneos de grau menor ou igual que $\delta$.

$a \cong b \quad a$ difeomorfo a $b, a, b \in\left[\Lambda^{2}\left(\mathbb{R}^{2}\right)\right]_{\{H=0\}}$. 


\section{Introdução}

As estruturas simpléticas surgem naturalmente em diversos contextos tais como: mecânica Hamiltoniana, teoria de campos, óptica geométrica, geometria algébrica, etc. Nestes casos, as bifurcações dos vários objetos simpléticos, como variedades Lagrangianas ou isotrópicas, representando as fases dos sistemas, desempenham um papel importante.

O problema da classificação simplética de curvas singulares foi introduzido por V.I. Arnold em [A]. Arnold mostrou que uma singularidade do tipo $A_{2 k}$ de uma curva plana (com relação ao grupo $\mathcal{A}$ ) se subdivide em $2 k+1$ órbitas com respeito à equivalência simplética. Ele distinguiu as diferentes singularidades simpléticas através do contato (ordem de tangência) da curva parametrizada com a subvariedade Lagrangiana suave mais próxima. Arnold expressou estes novos invariantes simpléticos em termos da interação da álgebra local com a estrutura simplética, chamada de álgebra local simplética.

Num espaço simplético $\left(\mathbb{R}^{2 n}, \omega\right)$ dizemos que dois conjuntos $N_{1}, N_{2}$ são simplectomorfos se existe um difeomorfismo que leva $N_{1}$ em $N_{2}$ e preserva $\omega$.

Neste trabalho consideramos $N$ uma curva algébrica singular plana quase-homogênea e queremos identificar dentre as curvas difeomorfas a $N$ aquelas que são simplectomorfas. Para isto usaremos o método das restrições algébricas. Este foi desenvolvido por W. Domitrz, S. Janeczko e M. Zhitomirskii em [D-J-Z], que é a nossa principal referência.

Esta disseratação está dividida em 5 capítulos. No Capítulo 1 introduzimos conceitos e resultados preliminares de variedades diferenciáveis, variedades simpléticas, teoria de singularidades e alguns conceitos de variedades algébricas, também descrevemos de maneira mas precisa o problema a ser resolvido (Problema A).

No Capítulo 2, definimos o $\mathbb{R}$-espaço vetorial das restrições algébricas de germes de $k$ formas diferenciáveis a um conjunto $N$ e obtemos a ação do grupo de simetrias locais de $N$ neste espaço vetorial. Como exemplo obtemos uma base do espaço das restrições 
algébricas no caso $N=\left\{A_{k}=0\right\}$ e as formas normais das órbitas com respeito à ação do grupo de simetrias locais. Reduzimos nosso Problema A ao problema de classificar as restrições algébricas no conjunto $\left[\operatorname{Symp}\left(\mathbb{R}^{2 n}\right)\right]_{N}$ com respeito ao grupo de simetrias locais de $N$ (Problema B) e revisamos vários teoremas dos quais resaltamos os Teoremas A e B, neste último temos condições necessárias e suficientes para quando um conjunto quase-homogêneo $N$ está contido numa variedade Lagrangiana.

No Capítulo 3, introduzimos o índice de isotropia e a multiplicidade simplética, invariantes simpléticos que foram introduzidos por Arnol'd. Obtemos os Teoremas C e D que mostram como calcular estes invariantes e alguns resultados relacionando os conjuntos $\left[\Lambda^{2}\left(\mathbb{R}^{2 n}\right)\right]_{N},\left[\Lambda^{2, \operatorname{closed}}\left(\mathbb{R}^{2 n}\right)\right]_{N}$ e $\left[\operatorname{Symp}\left(\mathbb{R}^{2 n}\right)\right]_{N}$.

No Capítulo 4, reduzimos nossos resultados para a variedade simplética $\left(\mathbb{R}^{2}, \omega\right)$, consideramos $H\left(x_{1}, x_{2}\right)$ polinômio quase-homogêneo, definimos a álgebra local e multiplicidade (número de Milnor) de $H$, as simetrias infinitesimais da curva $N=\{H=0\}(\operatorname{Derlog}\{N\})$ e encontramos o espaço tangente $T(a)$ à órbita de $a \in\left[\Lambda^{2}\left(\mathbb{R}^{2}\right)\right]_{N}$. Por último provamos os dois teoremas que serão fundamentais para a classificação em $\left[\Lambda^{2}\left(\mathbb{R}^{2}\right)\right]_{N}$ com respeito ao grupo de simetrias locais de $N$.

No Capítulo 5, como uma curta continuação do Capítulo 3 obtemos mais relações entre $\left[\Lambda^{2, \operatorname{closed}}\left(\mathbb{R}^{2 n}\right)\right]_{N}$ e $\left[\operatorname{Symp}\left(\mathbb{R}^{2 n}\right)\right]_{N}$. Obtemos a multiplicidade simplética e o índice de isotropia, a classificação de $\left[\Lambda^{2}\left(\mathbb{R}^{2}\right)\right]_{N}$ com respeito ao grupo de simetrias locais de $N$ e mediante esta classificação resolvemos o Problema A na variedade simplética $\left(\mathbb{R}^{2 n}, \omega\right), n \in$ $\mathbb{N}$. 


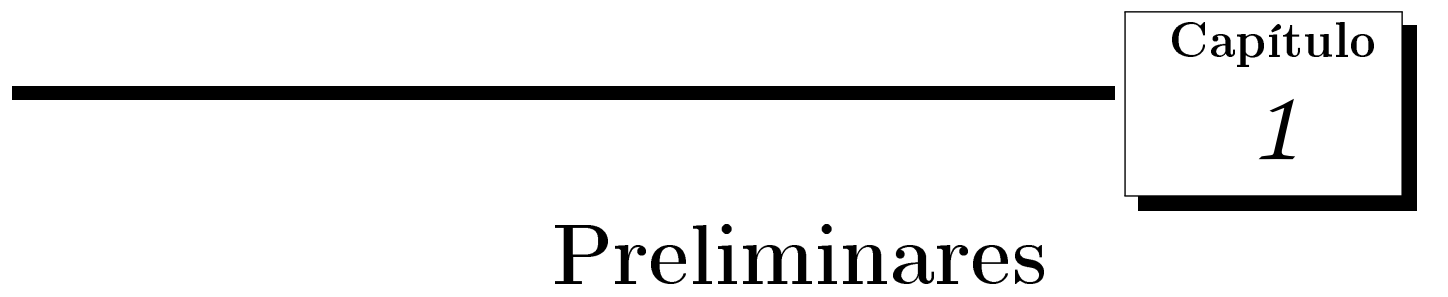

Como foi mencionado na Introdução neste capítulo apresentamos definições e resultados que serão a base para entender nosso problema (Problema A) a resolver assim como para desenvolver nos seguintes capítulos a teoria necessária para a sua resolução.

\subsection{Variedades Simpléticas}

Começamos supondo que definições como variedade suaves, funções suaves, sistema de coordenadas, derivada e espaço tangente são já conhecidas pelo leitor e podem ser encontradas por exemplo em [L].

Considere uma variedade suave $M$, lembre que se $p \in M$ então existe uma vizinhança $U$ de $p$ e uma função $\phi: U \longrightarrow \mathbb{R}^{n}$ que é difeomorfismo sobre a imagem. O par $(U, \phi)$ é chamado de carta ou sistema de coordenadas local do ponto $p$ e pelo geral a função $\phi$ será denotada por $\left(x_{1}, \ldots, x_{n}\right)$ (a função $\phi^{-1}$ é chamada de parametrização de $p$ em $M$ ) e o $n$ que existe não depende do ponto $p$ é chamado de dimensão da variedade, neste caso denotamos por $M^{n}$ para fazer ênfase em que $n$ é a dimensão da variedade $M$.

Sejam $M, N$ variedades suaves, $f: M \longrightarrow N$ é suave em $x \in M$ se para quaisquer $(U, \phi),(V, \psi)$ cartas de $x$ e $f(x)$ respectivamente tais que $f(U) \subset V$ temos que $\phi^{-1} \circ f \circ \phi$ : $\phi^{-1}(U) \longrightarrow \psi(V)$ é suave. No caso em que $f$ é suave em todo ponto de $M$ dizemos que $f$ é suave.

Definição 1.1: Seja $M$ uma variedade suave e seja $S \subset M$ um subconjunto. Dizemos que $S$ é uma subvariedade mergulhada ou simplesmente subvariedade, se $S$ com a topologia induzida de $M$ admite uma estrutura de variedade suave. No caso de $S$ ser uma subvariedade denotamos por $\operatorname{codim} S=\operatorname{dim} M-\operatorname{dim} S$ que é chamada de codimensão de 
$S$.

Proposição 1.2: Sejam $M^{m}$ variedade suave $e S \subset M$ uma subvariedade de dimensão s. Então para todo $p \in S$ existe uma carta local $(U, \phi)$ onde $\phi=\left(x_{1}, x_{2}, \ldots, x_{m}\right)$ tal que $\phi(U \cap S)$ é subconjunto de $\left\{\left(x_{1}, \ldots, x_{s}, x_{s+1}, \ldots, x_{m}\right) / x_{i}=0, s+1 \leqslant i \leqslant m\right\}$.

Dada uma variedade suave $M$ e $p \in M$ denotamos o espaço tangente a $M$ em por $T_{p} M$. Lembre que $T_{p} M$ é um $\mathbb{R}$ - espaço vetorial de dimensão $\operatorname{dim} M$. Sejam $M, N$ duas variedades e seja $f: M \longrightarrow N$ uma função suave, denotamos a derivada de $f$ em $p \in M$ por $d_{p} f: T_{p} M \longrightarrow T_{f(p)} N$.

\subsubsection{Campos vetoriais}

Esta subseção pode por exemplo ser encontrada em [T]. Assumimos que o leitor já esta familiarizado com fibrados vetoriais.

Lembramos que uma seção de um fibrado vetorial $\pi: E \longrightarrow M$ é uma função $s$ : $M \longrightarrow E$ tal que $s \circ \pi=i d_{M}$. Dizemos que a seção é suave quando $s$ for uma função suave. Também lembre que dada uma variedade suave $M$ o fibrado tangente de $M$ é

$$
T M=\bigcup_{p \in M}\{p\} \times T_{p} M
$$

Definição 1.3: Um campo vetorial ou campo de vetores na variedade suave $M$ é uma seção do fibrado vetorial $\pi: T M \longrightarrow M$, onde $\pi(p, v)=p, \forall p \in M, \forall v \in T_{p} M$.

Sejam $M$ uma variedade suave $\operatorname{com} \operatorname{dim} M=n$ e um campo de vetores $X$ de $M$. Então dada $\left(U,\left(x_{1}, \ldots, x_{n}\right)\right)$ uma carta local de $M$ em $p_{0}$, existem $a_{i}: U \longrightarrow \mathbb{R}, i=1, \ldots, n$ tais que

$$
X(p)=\left.\sum_{i=1}^{n} a_{i}(p) \frac{\partial}{\partial x_{i}}\right|_{p}, \quad \forall p \in U
$$

onde $\left\{\frac{\partial}{\partial x_{i}}\right\}_{1 \leqslant i \leqslant n}$ são os campos vetoriais tais que para todo ponto $p$ o conjunto $\left\{\left.\frac{\partial}{\partial x_{i}}\right|_{p}\right\}_{1 \leqslant i \leqslant n}$ é uma base do espaço $T_{p} M$.

Proposição 1.4: Seja X um campo de vetores na variedade suave $M$. Então $X$ é suave se e somente se para toda carta local $\left(U,\left(x_{1}, \ldots, x_{n}\right)\right)$ de $M$ se cumple que as funções $a_{i}$ dadas na equação (1.1) são suaves $\forall i=1, \ldots, n$.

Definição 1.5: Sejam $M$ uma variedade suave, $X$ um campo de vetores e $p \in M$. 
Dizemos que $X$ tem um fluxo local em $p$ se existe uma função suave

$$
\Phi:]-\varepsilon, \varepsilon[\times V \longrightarrow U, \varepsilon>0
$$

onde $\Phi(t, x)=\phi_{t}(x)$, tal que

1. $V \subset U \subset M$ são vizinhanças abertas de $p$;

2. $\Phi_{0}: V \longrightarrow U$ é a função inclusão;

3. $\Phi_{t_{1}+t_{2}}=\phi_{t_{1}} \circ \phi_{t_{2}}$, quando ambas expressões estão bem definidas;

4. $\left.\frac{\partial \Phi_{r}(q)}{\partial r}\right|_{r=t}=\left.\frac{d \Phi_{r}(q)}{d r}\right|_{r=t}=X_{\phi_{t}(q)}$, para todo $q \in V$.

Proposição $1.6([\mathrm{G}])$ : Seja $M$ uma variedade suave, $X$ um campo de vetores e $p \in M$. Então existe um fluxo local de $X$ em $p$.

\subsubsection{Formas diferenciáveis}

Esta subseção pode ser encontrada em qualquer dos textos citados anteriormente assim como em $[\mathrm{K}-\mathrm{M}]$, assumimos que o leitor já está familiarizado com o produto tensorial.

Seja $M^{n}$ numa variedade suave e $p \in M$. Uma $k$-forma em $p$ é uma função

$$
\omega_{p}: \underbrace{T_{p} M \times \ldots \times T_{p} M}_{k-\text { vezes }} \longrightarrow \mathbb{R}
$$

que é uma transformação $k$-linear (isto que dizer que é linear em cada componente) e que é alternada isto é $\omega_{p}\left(\ldots, v_{i}, \ldots, v_{j}, \cdots\right)=-\omega_{p}\left(\ldots, v_{j}, \ldots, v_{i}, \cdots\right)$ para $v_{i}, v_{j} \in T_{p} M, i \neq j$.

O Conjunto de todas as $k$-formas em $p$ é denotado por $\Lambda^{k}\left(T_{p} M\right)$. O conjunto

$$
\Lambda_{*}^{k}(T M)=\bigcup_{p \in M}\{p\} \times \Lambda^{k}\left(T_{p} M\right)
$$

é um fibrado vetorial chamado de fibrado das $k$-formas. Uma seção suave de $\Lambda_{*}^{k}(T M)$ é chamado de $k$-forma diferenciável em $M$. O conjunto das $k$-formas diferenciáveis em $M$ é um $\mathbb{R}$-espaço vetorial e é denotado por $\Lambda^{k}(M)$. As 0-formas diferenciáveis são justamente as funções suaves em $M$.

Seja $k \in \mathbb{N}$, denotamos por $S_{k}$ o grupo de permutações de $k$ elementos, para cada $\sigma \in S_{k}$ temos associado um valor $\operatorname{sign}(\sigma)$ que é 1 ou -1 . Seja $V$ um espaço vetorial e $T: V \times \ldots \times V \longrightarrow \mathbb{R}$ uma transformação $k$-linear, definimos a operação alternação de T como

$$
\operatorname{Alt}(T)\left(v_{1}, \ldots, v_{k}\right)=\frac{1}{k !} \sum_{\sigma \in S_{k}} \operatorname{sign}(\sigma) T\left(v_{\sigma(1)}, \ldots, v_{\sigma(k)}\right) .
$$


Sejam $k, l \in \mathbb{N}$ definimos o produto exterior

$$
\wedge: \Lambda^{k}\left(T_{p} M\right) \times \Lambda^{l}\left(T_{p} M\right) \longrightarrow \Lambda^{k+l}\left(T_{p} M\right)
$$

por $\omega \wedge \alpha=\frac{(k+l) !}{k ! l !} A l t(\omega \otimes \alpha)$, onde $\otimes$ denota o produto tensorial.

Seja $M$ uma variedade suave, $p \in M$, considere uma carta $\left(U,\left(x_{1}, \ldots, x_{n}\right)\right)$ de $M$ em $p$, denotamos por $\left.d x_{i}\right|_{p}$ ou $d x_{i}$ quando não haver confusão do ponto de $M i=1, \ldots, n$ as 1-formas em $p$ tais que

$$
\left.d x_{i}\right|_{p}\left(\left.\frac{\partial}{\partial x_{i}}\right|_{p}\right)= \begin{cases}1, & i=j \\ 0, & i \neq j\end{cases}
$$

Sejam $v_{i}=\left.\sum_{j=1}^{n} a_{i j} \frac{\partial}{\partial x_{i}}\right|_{p}, i=1, \ldots, k$, então

$$
d x_{i_{1}} \wedge \ldots \wedge d x_{i_{k}}\left(v_{1}, \ldots, v_{k}\right)=\operatorname{det}\left(\begin{array}{cccc}
a_{i_{1} 1} & a_{i_{2} 1} & \cdots & a_{i_{k} 1} \\
a_{i_{1} 2} & a_{i_{2} 2} & \cdots & a_{i_{k} 2} \\
\vdots & \vdots & \ddots & \vdots \\
a_{i_{1} k} & a_{i_{2} k} & \cdots & a_{i_{k} k}
\end{array}\right)
$$

Proposição 1.7: Seja $M$ uma variedade suave, $\omega \in \Lambda_{*}^{k}(T M), \eta_{1}, \eta_{2} \in \Lambda_{*}^{l}(T M), \gamma \in$ $\Lambda_{*}^{m}(T M)$ então temos

1. $\omega \wedge\left(\eta_{1}+\eta_{2}\right)=\omega \wedge \eta_{1}+\omega \wedge \eta_{2}$

2. $\omega \wedge \eta_{1}=(-1)^{k l} \eta \wedge \omega$;

3. $\omega \wedge(\eta \wedge \gamma)=(\omega \wedge \eta) \wedge \gamma$.

Proposição 1.8: Sejam $M$ uma variedade suave, $p \in M$ e $\omega$ uma k-forma diferenciável. Então dada uma carta $\left(U,\left(x_{1}, \ldots, x_{n}\right)\right)$ de $M$ em $p$ existem funções suaves $f_{I}=a_{i_{1} i_{2} \ldots i_{k}}$ : $U \longrightarrow \mathbb{R}, 1 \leqslant i_{1}<\ldots<i_{k} \leqslant n$ tais que para todo $q \in U$ temos

$$
\omega_{q}=\omega(q)=\sum_{I} f_{I}(q) d x_{I}, \text { onde } d x_{I}=d x_{i_{1}} \wedge \ldots \wedge d x_{i_{n}}
$$

Definição 1.9: Sejam $M, N$ variedades suaves, $f: M \longrightarrow N$ uma função suave, definimos o pull-back $f^{*}: \Lambda^{k}(N) \longrightarrow \Lambda^{k}(M)$ dada por

$$
\left(f^{*} \omega\right)_{p}\left(v_{1}, \ldots, v_{k}\right)=\omega_{f(p)}\left(d_{p} f\left(v_{1}\right), \ldots, d_{p} f\left(v_{k}\right)\right), \text { para } v_{1}, \ldots, v_{k} \in T_{p} M
$$

Observação 1.10: Note que na Definição 1.9 , se $k=0$, então $f^{*}(g)=g \circ f$.

Proposição 1.11: Nas condições da definição 1.9, sejam $g: N \longrightarrow P$ função suave, $\omega_{1}, \omega_{2} \in \Lambda^{k}(M)$ e $\eta \in \Lambda^{l}(M)$, então 
1. $f^{*}\left(\omega_{1}+\omega_{2}\right)=f^{*}\left(\omega_{1}\right)+f^{*}\left(\omega_{2}\right)$;

2. $f^{*}\left(\omega_{1} \wedge \eta\right)=f^{*}\left(\omega_{1}\right) \wedge f^{*}(\eta)$;

3. $(g \circ f)^{*}\left(\omega_{1}\right)=f^{*} g^{*}\left(\omega_{1}\right)$.

\subsubsection{Derivada de Lie}

Esta subseção pode ser encontrada em [L].

Definição 1.12: Seja $V$ um campo de vetores na variedade $M$, definimos o produto interior $i_{V}: \Lambda^{k}(M) \longrightarrow \Lambda^{k-1}(M)$ por $i_{V}(\omega)\left(p, v_{1}, \ldots, v_{k-1}\right)=\omega\left(p, V_{p}, v_{1}, \ldots, v_{k-1}\right)=$ $\omega_{p}\left(V_{p}, v_{1}, \ldots, v_{k-1}\right)$ onde $V_{p}=V(p), v_{1}, \ldots, v_{k-1} \in T_{p} M$.

Definição 1.13: Sejam $M^{n}$ uma variedade diferenciável e $k \in \mathbb{N}_{0}$. Uma derivada exterior $d: \Lambda^{k}(M) \longrightarrow \Lambda^{k+1}(M)$ é uma função que satistfaz:

1. $d$ é linear sobre $\mathbb{R}$.

2. Se $\omega \in \Lambda^{k}(M)$ e $\alpha \in \Lambda^{l}(M)$, então

$$
d(\omega \wedge \alpha)=d(\omega) \wedge \alpha+(-1)^{k l} \omega \wedge d(\alpha) .
$$

3. $d \circ d=0$.

4. Para $f \in \Lambda^{0}(M), d f$ é a diferencial de $f$ dada por $d f(X)=X(f)$, onde se $X=$ $\sum_{i=1}^{n} g_{i} \frac{\partial}{\partial x_{i}}$ é um campo de vetores então $X(f)=\sum_{i=1}^{n} g_{i} \frac{\partial f}{\partial x_{i}}$.

Proposição 1.14: Sejam $M^{n}$ uma variedade diferenciável, então existe uma única derivada exterior e ela é denotada por d.

Proposição 1.15: Dado um sistema de coordenadas $\left(U,\left(x_{1}, \ldots x_{n}\right)\right)$ da variedade suave $M^{n}$, se $\omega=\sum_{I} a_{I} d x_{I}$, então $d(\omega)=\sum_{I} d\left(a_{I}\right) \wedge d x_{I}$, onde $d\left(a_{I}\right)$ é a diferencial da função $a_{I}$.

Proposição 1.16: Seja $F: M \longrightarrow N$ uma função suave entre variedades suaves, então $F^{*}: \Lambda^{k}(N) \longrightarrow \Lambda^{k}(M)$ comuta com d: para todo $\omega \in \Lambda^{k}(N)$ temos $F^{*}(d \omega)=d\left(F^{*} \omega\right)$.

Definição 1.17: Uma $k$ forma diferenciável $\omega \in \Lambda^{k}(M)$ é chamada fechada se $d(\omega)=0$ e é chamada exata se existe $\alpha \in \Lambda^{k-1}(M)$ tal que $d(\alpha)=\omega$. 
Definição 1.18: Seja $X$ um campo de vetores e $\omega$ uma $k$-forma diferenciável na variedade suave $M$, a derivada de Lie $\mathscr{L}_{X}: \Lambda^{k}(M) \longrightarrow \Lambda^{k}(M)$, em $p \in M$ é definida por

$$
\left(\mathscr{L}_{X} \omega\right)_{p}=\left(\mathscr{L}_{X} \omega\right)(p)=\lim _{t \rightarrow 0} \frac{\left(\Phi_{t}^{*} \omega\right)_{p}-\omega_{p}}{t}=\left.\frac{d}{d t}\left(\Phi_{t}^{*} \omega\right)\right|_{p},
$$

onde $\Phi$ é um fluxo de $X$ em $p$.

Observação 1.19: A definição 1.18 não depende do fluxo local $\Phi$.

Proposição 1.20: Seja X um campo de vetores na variedade suave $M$. Então temos as seguintes propriedades:

1. $\mathscr{L}_{X} f=X(f)$, f função suave;

2. $\mathscr{L}_{X}(\omega \wedge \beta)=\left(\mathscr{L}_{X} \omega\right) \wedge \beta+\omega \wedge\left(\mathscr{L}_{X} \beta\right), \forall \omega \in \Lambda^{k}(M), \beta \in \Lambda^{l}(M)$;

3. $\mathscr{L}_{X} d \omega=d \mathscr{L}_{X} \omega, \forall \omega \in \Lambda^{k}(M)$;

4. (Fórmula de Cartan) $\mathscr{L}_{X} \omega=d i_{X} \omega+i_{X} d \omega, \forall \omega \in \Lambda^{k}(M)$.

Proposição $\mathbf{1 . 2 1}$ (ver $[\mathrm{S}]$ ): Sejam $M$ uma variedade suave, $I \subset \mathbb{R}$ um intervalo aberto que contém o zero. Seja $\left\{\omega_{t}, t \in I\right\}$ uma família de $k$-formas, $\left\{X_{t}, t \in I\right\}$ uma família de campos vetoriais e $\left\{\rho_{t}, t \in I\right\}$ uma familia de difeomorfismos de $M$ tais que são suaves com respeito a te $\left.\frac{\partial \rho_{r}}{\partial r}\right|_{r=t}=X_{t} \circ \rho_{t}$. Então

$$
\frac{d}{d t} \rho_{t}^{*} \omega_{t}=\rho_{t}^{*}\left(\mathscr{L}_{X_{t}} \omega_{t}+\frac{d \omega_{t}}{d t}\right)
$$

\subsubsection{Variedades Simpléticas}

A seguinte subseção pode ser encontrada em [S].

Definição 1.22: Sejam $M$ uma variedade suave e $\omega$ uma 2-forma diferenciável em $M$. Então $(M, \omega)$ é chamado de variedade simplética ou espaço simplético se $\omega$ satisfaz:

1. $\omega$ é uma forma fechada;

2. Para cada $p \in M, \omega_{p}$ é anti-simetrica, isto é, $\omega_{p}(v, w)=-\omega_{p}(w, v)$, para todo $v, w \in T_{p} M$

3. Para cada $p \in M$, se $v \in T_{p} M$ é tal que $\omega_{p}(v, w)=0, \forall w \in T_{p} M$, então $v=0$. 
Proposição 1.23: Se $(M, \omega)$ é uma variedade simplética, então $\operatorname{dim} M=2 n$, para algum $n \in \mathbb{N}$.

Exemplo 1.24: Sejam $M=\mathbb{R}^{2 n}$ com coordenadas $x_{1}, \ldots, x_{n}, y_{1}, \ldots \ldots, y_{n}$ e a 2 -forma diferenciável $\omega_{0}=\sum_{i=1}^{n} d x_{i} \wedge d y_{i}$. Então $\left(M, \omega_{0}\right)$ é uma variedade simplética.

Definição 1.25: Sejam $\left(M_{1}, \omega_{1}\right),\left(M_{2}, \omega_{2}\right)$ duas variedades simpléticas $2 n$-dimensionais e $\phi: M_{1} \longrightarrow M_{2}$ um difeomorfismo. Então $\phi$ é chamado simplectomorfismo se $\phi^{*}\left(\omega_{2}\right)=\omega_{1}$.

Teorema 1.26 (Clássico de Darboux): Seja $(M, \omega)$ uma variedade simplética $2 n$-dimensional, e seja $p \in M$. Então existe um sistema de coordenadas $\left(U,\left(x_{1}, \ldots, x_{n}, y_{1}, \ldots, y_{n}\right)\right)$ de $M$ em $p$ tal que em $U$ temos

$$
\omega=\sum_{i=1}^{n} d x_{i} \wedge d y_{i}
$$

A carta $\left(U,\left(x_{1}, \ldots, x_{n}, y_{1}, \ldots, y_{n}\right)\right)$ no Teorema 1.26 é chamada de Carta de Darboux.

Definição 1.27: Seja $(M, \omega)$ uma variedade simplética $2 n$-dimensional, uma subvariedade $N \subset M$ é chamada isotrópica com respeito a $\omega$ se $i_{N}: N \longrightarrow M$, a função inclusão, satisfaz $i_{N}^{*}(\omega)=0$, isto quer dizer que a forma $\omega$ se anula em $N$.

Proposição 1.28: Nas condições da Definição 1.27 temos que se a subvariedade $N \subset M$ é isotrópica com respeito a $\omega$ então $\operatorname{dim} N \leqslant n$.

Definição 1.29: Uma subvariedade isotrópica $N$ tal que $\operatorname{dim} N=\frac{\operatorname{dim} M}{2}$ é chamada de subvariedade Lagrangiana.

\subsection{Teoria de Singularidades}

Esta seção pode ser encontrada por exemplo em [G].

\subsubsection{Germes e Jatos}

Definição 1.30: Seja $M$ um subconjunto de um espaço topológico e $p \in M$. Dizemos que dois subconjuntos $A, B \subset M$ são equivalentes em $p \in A \cap B$ se existe uma vizinhança aberta $W$ de $p$ tal que $W \cap A=W \cap B$.

Observe que a relação anterior é de equivalência no conjunto das partes de $M$. As classes de equivalência são chamadas de germes de conjuntos. No caso de $M$ ser uma 
variedade diferenciável as classes de equivalência são chamadas de germes de variedades e são denotadas por $(M, p)$.

Sejam $M, N$ duas variedades suaves. Consideremos o conjunto das funções suaves definidas em uma vizinhança de $x \in M$ com valores em $N$.

$\{(U, g) / U$ é vizinhaça aberta de $x$ em $M$ e $g: U \longrightarrow N$ suave $\}$.

Neste conjunto introduzimos a seguinte relação de equivalência: $f: U \longrightarrow N$ e $g$ : $V \longrightarrow N$, onde $U, V$ são vizinhanças de $x$ em $M$, são equivalentes se existe uma vizinhança aberta $W$ de $x$ em $M$ tal que $W \subset U \cap V$ e $\left.f\right|_{W}=\left.g\right|_{W}$.

As classes de equivalência são chamadas germes em $x$ de funções suaves de $M$ em $N$ e denotadas por $f:(M, x) \longrightarrow(N, y)$. O $x$ é chamado de fonte e $y$ chamado de meta.

Seja $f:(M, x) \longrightarrow(N, y)$ um germe de uma função suave. Definimos o posto de $f$ como o posto de $d f_{x}$ onde $f$ é um representante do germe.

Definição 1.31: Sejam $M, N$ e $P$ variedades suaves, $f:(M, x) \longrightarrow(N, y)$ e $g$ : $(N, y) \longrightarrow(P, z)$ germes de funções suaves com representantes $f: U \longrightarrow N$ e $g: V \longrightarrow P$ respectivamente tais que $f(U) \subset V$. Definimos $g \circ f:(M, x) \longrightarrow(P, z)$ como a classe de equivalência de $g \circ f: U \longrightarrow P$.

Definição 1.32: Sejam $M, N$ variedades suaves, dizemos que um germe $f:(M, x) \longrightarrow$ $(N, y)$ é invertível se existir $g:(N, y) \longrightarrow(M, x)$ tal que $f \circ g$ é o germe da identidade em $(N, y)$ e $g \circ f$ é o germe da identidade em $(M, x)$. Neste caso dizemos que o germe $f$ é um germe de difeomorfismo.

Segue do Teorema da função inversa para variedades suaves o seguinte resultado.

Teorema 1.33: Seja $M, N$ variedades suaves, $x \in M$ e $y \in N$. Um germe $f:(M, x) \longrightarrow$ $(N, y)$ é invertivel se e somente $d f_{x}: T_{x} M \longrightarrow T_{y} N$ é isomorfismo.

Denotamos por $J^{k}(n, p)$ o espaço vetorial das funções suaves $f: \mathbb{R}^{n} \longrightarrow \mathbb{R}^{p}, f=$ $\left(f_{1}, \ldots, f_{p}\right)$, onde cada componente $f_{i}, i=1, \ldots, p$, é um polinômio de grau menor ou igual que $k$ nas coordenadas $x_{1}, x_{2}, \ldots, x_{n}$ de $\mathbb{R}^{n}$, com termo constante nulo. Os elementos de $J^{k}(n, p)$ são chamados de $k$-jatos. Notemos que $J^{k}(n, p)$ é uma variedade.

Definição 1.34: Seja $f: \mathbb{R}^{n} \longrightarrow \mathbb{R}^{p}$ uma função suave e $a \in \mathbb{R}^{n}$. Definimos a aplicação $j^{k} f(a): \mathbb{R}^{n} \longrightarrow J^{k}(n, p)$, onde $j^{k} f(a)$ é o polinômio de Taylor de $f(x+a)-f(a)$ de ordem 
$k$ na origem. A aplicação $j^{k} f$ é suave e $j^{k} f(a)$ é chamado de $k$-jato de $f$ em $a$,

$$
j^{k} f(a)=d f_{a}(x)+\frac{1}{2} d^{2} f_{a}(x, x)+\ldots+\frac{1}{k !} d^{k} f_{a}(x, \ldots, x) .
$$

Observação 1.35: Seja $a \in \mathbb{R}^{n}$ e uma função suave $f: \mathbb{R}^{n} \longrightarrow \mathbb{R}^{p}$. Denotamos $j^{0} f(a)=$ $f(a)$ e chamamos este de 0 -jato de $f$ em $a$.

\subsubsection{Ação de Grupo}

Sejam $G$ um grupo e $M$ um conjunto. Uma ação de $G$ em $M$ é uma função $\phi$ : $G \times M \longrightarrow M$, denotada por $g \cdot x=\phi(g, x)$, satisfazendo:

1. $1 \cdot x=x, \forall x \in M$ e onde 1 denota o elemento identidade de $G$.

2. $(g \cdot h) \cdot x=g \cdot(h \cdot x), \forall g, h \in G, \forall x \in M$.

Definição 1.36: Dada uma ação podemos definir uma relação de equivalência em $M$ : se $x, y \in M, x$ é equivalênte a $y$ se existe $g \in G$ tal que $y=g \cdot x$. As classes de equivalência são chamadas órbitas.

Se $x \in M$, a órbita de $x$ é denotada por

$$
G \cdot x=\{g \cdot x / g \in G\}
$$

Exemplo 1.37: Seja $G L(n)$ o grupo das transformações lineares invertíveis em $\mathbb{R}^{n}$ (matrizes invertíveis) com a operação de composição e $M=J^{k}(n, p)$. Uma ação $\phi$ : $G L(n) \times G L(p) \times M \longrightarrow M$ do grupo $G L(n) \times G L(p)$ em $M$ é definida da seguinte maneira:

$$
\phi((H, K), f)=K \circ f \circ H^{-1}
$$

Definição 1.38: Um grupo de Lie $G$ é um grupo que é uma variedade suave e as funções de multiplicação $G \times G \longrightarrow G$ definida por $(g, h) \longmapsto g h$ e inversão $G \longrightarrow G$ definida por $g \longmapsto g^{-1}$ são suaves.

Proposição 1.39: Seja $\phi: G \times M \longrightarrow M$ uma ação do grupo de Lie $G$ numa variedade suave $M$. Suponhamos que as órbitas sejam subvariedades. Seja $x \in M$, então $\phi_{x}: G \longrightarrow$ $G \cdot x$ dada por $\phi_{x}(g)=g \cdot x$ é uma submersão.

Corolário 1.40: Nas hipóteses da Proposiçẫo 1.39 temos que $T_{x} G \cdot x=d \phi_{x_{1}}\left(T_{1} G\right)$.

Lema 1.41 (de Mather): Seja $G$ um grupo de Lie agindo numa variedade suave $M$ e $N \subset M$ uma subvariedade conexa. Suponhamos que as órbitas sejam subvariedades. Então $N$ está contida numa unica órbita se e somente se, 
1. $T_{y} N \subset T_{y} G \cdot y, \quad \forall y \in N$.

2. $\operatorname{dim} T_{y} G \cdot y=$ constante, $\forall y \in N$.

Exemplo 1.42: Seja $M=H^{d}(n, p)$ o subespaço vetorial de $J^{d}(n, p)$ constituído das funções cujas componentes são polinômios homogêneos de grau $d$ nas coordenadas $x_{1}, \ldots, x_{n}$ de $\mathbb{R}^{n}$, seja $G=G L(n) \times G L(p)$ e seja $\phi$ a ação dada no Exemplo 1.37. Então temos

$$
T_{f} G \cdot f=\mathbb{R}\left\{\frac{\partial f}{\partial x_{i}} x_{j}\right\}_{i, j=1, \ldots, n}+\mathbb{R}\{\underbrace{\left(0, \ldots, f_{j}, 0, \ldots, 0\right)}_{f_{j} \text { no } i \text { ésimo lugar }}\}_{i=1, \ldots, n}^{j=1, \ldots, p} .
$$

\subsubsection{A álgebra $\varepsilon_{n}$}

Sejam $M$ uma variedade e $x \in M$. Estamos interessados em germes de funções suaves $f:(M, x) \longrightarrow(\mathbb{R}, y)$. Mediante uma parametrização de $M$ em $x$ podemos supor $M=\mathbb{R}^{n}$ e $x=0$. Definimos

$$
\varepsilon_{n}=\left\{f:\left(\mathbb{R}^{n}, 0\right) \longrightarrow(\mathbb{R}, y) / f \text { é germe de função suave }\right\} .
$$

Temos que $\varepsilon_{n}$ é um anel local cujo ideal maximal é

$$
\mathscr{M}_{n}=\left\{f \in \varepsilon_{n} / f(0)=0\right\} .
$$

Definição 1.43: Seja $k \in \mathbb{N}$, denotamos por $J_{k}$ o ideal de $\varepsilon_{n}$ que consiste das funções que tem $(k-1)$-jato zero na origem, isto é

$$
J_{k}=\left\{f \in \varepsilon_{n} / j^{k-1} f(0)=0\right\}
$$

Lema 1.44 (de Hadamard): Sejam $U \subset \mathbb{R}^{n}$ vizinhança convexa de $0, f: U \times \mathbb{R}^{q} \longrightarrow \mathbb{R}$ função suave tal que $f(0, y)=0, \forall y \in \mathbb{R}^{q}$, então existem funções $f_{1}, \ldots, f_{n}: U \times \mathbb{R}^{q} \longrightarrow \mathbb{R}$ tais que $f=x_{1} f_{1}+\ldots+x_{n} f_{n}$ onde $x_{1}, \ldots, x_{n}$ são as funções coordenadas de $\mathbb{R}^{n}$.

Observação 1.45: No caso em que $q=0$, com o Lema de Hadamard se demonstra que $\mathscr{M}_{n}=\left\langle x_{1}, \ldots, x_{n}\right\rangle$ e $J_{k}=\mathscr{M}_{n}^{k}$.

Lema 1.46 (de Nakayama): Seja $R$ anel comutativo com unidade 1. Seja $m \subset R$ ideal tal que $1+x$ é invertivel $\forall x \in m$. Seja $M$ um $R$-módulo e $A, B \subset M R$-submódulos com $A$ finitamente gerado. Se $A \subset B+m A$ então $A \subset B$.

Seja $f \in \varepsilon_{n}$, denote ao ideal gerado pelas derivadas parciais de $f$ por

$$
\langle\nabla f\rangle=\left\langle\frac{\partial f}{\partial x_{1}}, \ldots, \frac{\partial f}{\partial x_{n}}\right\rangle .
$$


Temos que $\varepsilon_{n}$ é um $\varepsilon_{n}$-módulo e $\langle\nabla f\rangle$ é um $\varepsilon_{n}$-submódulo portanto o quociente $\frac{\varepsilon_{n}}{\langle\nabla f\rangle}$ é um $\mathbb{R}$-espaço vetorial. Definimos o número de Milnor de $f$ por

$$
\mu(f)=\operatorname{dim}_{\mathbb{R}} \frac{\varepsilon_{n}}{\langle\nabla f\rangle} .
$$

Proposição 1.47: Seja $f \in \varepsilon_{n}$ tal que $\mu=\mu(f)<\infty$ então $\mathscr{M}_{n}^{\mu} \subset\langle\nabla f\rangle$.

Demonstração: Considere a sequência de submódulos

$$
\varepsilon_{n}=\varepsilon_{n}+\langle\nabla f\rangle \supset \mathscr{M}_{n}+\langle\nabla f\rangle \supset \mathscr{M}_{n}^{2}+\langle\nabla f\rangle \supset \ldots \supset \mathscr{M}_{n}^{\mu}+\langle\nabla f\rangle \supset \ldots \supset\langle\nabla f\rangle
$$

que induz as desigualdades

$$
\begin{aligned}
0=\operatorname{dim}_{\mathbb{R}} \frac{\varepsilon_{n}}{\varepsilon_{n}} \leqslant \operatorname{dim}_{\mathbb{R}} \frac{\varepsilon_{n}}{\langle\nabla f\rangle+\mathscr{M}_{n}} & \leqslant \operatorname{dim}_{\mathbb{R}} \frac{\varepsilon_{n}}{\langle\nabla f\rangle+\mathscr{M}_{n}^{2}} \leqslant \\
& \leqslant \ldots \leqslant \operatorname{dim}_{\mathbb{R}} \frac{\varepsilon_{n}}{\langle\nabla f\rangle+\mathscr{M}_{n}^{\mu}} \leqslant \ldots \leqslant \operatorname{dim}_{\mathbb{R}} \frac{\varepsilon_{n}}{\langle\nabla f\rangle}=\mu<\infty
\end{aligned}
$$

Note que cada inclusão estrita na sequência 1.4 contribui com no mínimo 1 para o cálculo da dimensão, portanto existe $i \in \mathbb{N}, i \leqslant \mu$ tal que $\mathscr{M}_{n}^{i}+\langle\nabla f\rangle=\mathscr{M}_{n}^{i+1}+\langle\nabla f\rangle$ que implica $\mathscr{M}_{n}^{i} \subset \mathscr{M}_{n} \mathscr{M}_{n}^{i}+\langle\nabla f\rangle$ assim pelo Lema de Nakayama $\mathscr{M}_{n}^{i} \subset\langle\nabla f\rangle$ consequentemente $\mathscr{M}_{n}^{\mu} \subset \mathscr{M}_{n}^{i} \subset\langle\nabla f\rangle$.

Dada $f \in \varepsilon_{n}$ temos sua série de Taylor em torno de zero

$$
\hat{f}=f(0)+\sum_{i=1}^{n} \frac{\partial f}{\partial x_{i}}(0) x_{i}+\frac{1}{2} \sum_{i, j=1}^{n} \frac{\partial^{2} f}{\partial x_{i} \partial x_{i}}(0) x_{i} x_{j}+\cdots
$$

Assim definimos o homomorfismo de $\mathbb{R}$-álgebras $\phi: \varepsilon_{n} \longrightarrow \mathbb{R}\left[\left[x_{1}, \ldots, x_{n}\right]\right]$ dada por $\phi(f)=\hat{f}$. Se $f \in \varepsilon_{n}$ denotamos por $\langle\hat{\nabla f}\rangle=\phi(\langle\nabla f\rangle)$ e a dimensão $\operatorname{dim}_{\mathbb{R}} \frac{\mathbb{R}\left[\left[x_{1}, \ldots, x_{n}\right]\right]}{\langle\hat{\nabla f}\rangle}$ é chamada de codimensão de $\langle\hat{\nabla f}\rangle$, denotada por $\operatorname{cod}(\langle\hat{\nabla} f\rangle)$.

Proposição 1.48: Seja $f \in \varepsilon_{n}$, então $\mu(f)<\infty$ se e somente se $\operatorname{cod}(\langle\hat{\nabla} f\rangle)<\infty$. Além disso, se $\mu(f)<\infty$ temos $\mu(f)=\operatorname{cod}(\langle\hat{\nabla} f\rangle)$.

Um germe $f:\left(\mathbb{R}^{n}, 0\right) \longrightarrow\left(\mathbb{R}^{p}, 0\right)$ induz um homomorfismo de $\mathbb{R}$-álgebras $f^{*}: \varepsilon_{p} \longrightarrow$ $\varepsilon_{p}$ definida por $f^{*}(g)=g \circ f$ e é chamado de homomorfismo induzido por $f$. Sejam $f:\left(\mathbb{R}^{n}, 0\right) \longrightarrow\left(\mathbb{R}^{p}, 0\right)$ e $g:\left(\mathbb{R}^{p}, 0\right) \longrightarrow\left(\mathbb{R}^{k}, 0\right)$ germes de funções suaves, então $(g \circ f)^{*}=$ $f^{*} \circ g^{*}$.

Proposição 1.49: Seja $f:\left(\mathbb{R}^{n}, 0\right) \longrightarrow\left(\mathbb{R}^{n}, 0\right)$. Então $f^{*}$ é isomorfismo se e somente se $f$ é germe de difeomorfismo. Além disso $f^{-1^{*}}=f^{*-1}$. 


\subsubsection{Germes Simples}

Seja $D_{n}=\left\{h:\left(\mathbb{R}^{n}, 0\right) \longrightarrow\left(\mathbb{R}^{n}, 0\right) / h\right.$ é um germe de difeomorfismo $\}$. O conjunto $D_{n}$ é um grupo com a operação de composição e age em $\varepsilon_{n}$ da seguinte maneira: $\phi: D_{n} \times \varepsilon_{n} \longrightarrow$ $\varepsilon_{n}$ é dada por $\phi(h, f)=f \circ h^{-1}$. Denotamos o grupo $D_{n}$ com tal ação por $\mathcal{R}$.

Definição 1.50: Sejam $f, g \in \varepsilon_{n}$. Dizemos que $f$ é $\mathcal{R}$-equivalente a $g$ se existe $h \in D_{n}$ tal que $g=f \circ h^{-1}$.

Definimos $D_{n}^{k}=\left\{j^{k} h(0) / h \in D_{n}\right\} \subset J^{k}(n, n) . D_{n}^{k}$ é um grupo de Lie com a operação:

$$
j^{k} f(0) * j^{k} g(0)=j^{k}(f \circ g)(0)
$$

e age em $J^{k}(n, p)$ com a ação $\phi: D_{n}^{k} \times J^{k}(n, p) \longrightarrow J^{k}(n, p)$ dada por $\phi(h, f)=j^{k}(f \circ$ $\left.h^{-1}\right)(0)$. Denotamos o grupo $D_{n}^{k}$ com tal ação por $\mathcal{R}^{k}$.

Proposição 1.51: Sejam $f, g \in \varepsilon_{n}$ tais que $f$ e $g$ são $\mathcal{R}$-equivalentes, então $\langle\nabla f\rangle$ e $\langle\nabla g\rangle$ são isomorfos. Segue que se $\mu(f)<\infty$ então $\mu(f)=\mu(g)$.

A Proposição 1.51 diz que o número de Milnor é um invariante das órbitas da $\mathcal{R}$ equivalência.

Definição 1.52: Dizemos que $f \in \varepsilon_{n}$ é $k$-determinado se todo $g \in \varepsilon_{n}$ tal que $j^{k} f(0)=$ $j^{k} g(0)$ é $\mathcal{R}$-equivalente a $f$. Dizemos que $f$ é finitamente determinado se $f$ é $k$-determinado para algum $k \in \mathbb{N}$.

Proposição 1.53: Se $f \in \varepsilon_{n}$ é tal que $\mathscr{M}_{n}^{k} \subset \mathscr{M}_{n}\langle\nabla f\rangle$ para algum $k$, então $f$ é $k$ determinado.

Segue das Proposições 1.53 e 1.47 o seguinte corolario.

Corolário 1.54: Se $f \in \varepsilon_{n}$ é tal que $\mu=\mu(f)<\infty$ então $f$ é $(\mu+1)$-determinado.

Definição 1.55: Seja $G$ um grupo de Lie agindo numa variedade $M$. Dizemos que uma órbita $G \cdot x$ é simples se existe uma vizinhança suficientemente pequena de $g_{0} \cdot x$, para algum $g_{0} \in G$, que encontra somente um número finito de órbitas.

Definição 1.56: Um germe é dito simples se é $k$-determinado para algum $k \in \mathbb{N}$ e seu $k$ jato como elemento de $J^{N}(n, 1), N \geqslant k$, é simples, isto é tem uma vizinhaça que encontra somente um número finito de $\mathcal{R}^{N}$-órbitas. 
Teorema 1.57 (Arnold): Um germe simples $f \in \mathscr{M}_{n}^{2}$ é $\mathcal{R}$-equivalente a um dos seguintes germes.

1. $A_{k}: \pm x_{1}^{k+1} \pm x_{2}^{2} \pm \ldots \pm x_{n}^{2}, k \geqslant 1$;

2. $D_{k}: x_{1} x_{2}^{2} \pm x_{2}^{k-1} \pm x_{3}^{2} \pm \ldots \pm x_{n}^{2}, k \geqslant 4$;

3. $E_{6}: x_{1}^{3} \pm x_{2}^{4} \pm x_{3}^{2} \pm \ldots \pm x_{n}^{2}$;

4. $E_{7}: x_{1}^{3}+x_{1} x_{2}^{3} \pm x_{3}^{2} \pm \ldots \pm x_{n}^{2}$;

5. $E_{8}: x_{1}^{3}+x_{2}^{5} \pm x_{3}^{2} \pm \ldots \pm x_{n}^{2}$.

\subsection{5 $\mathcal{C}, \mathcal{K}$-equivalência}

Definimos $\varepsilon_{n, p}$ como o conjunto dos germes $f:\left(\mathbb{R}^{n}, 0\right) \longrightarrow\left(\mathbb{R}^{p}, y\right)$ e $\mathscr{M}_{n} \varepsilon_{n, p}$ como o conjunto de germes $f:\left(\mathbb{R}^{n}, 0\right) \longrightarrow\left(\mathbb{R}^{p}, 0\right)$.

Temos que $\varepsilon_{n, p}$ é um $\varepsilon_{n}$-módulo e $\mathscr{M}_{n} \varepsilon_{n, p}$ é um $\varepsilon_{n}$-submódulo de $\varepsilon_{n, p}$.

Definição 1.58: Dizemos que $f, g \in \mathscr{M}_{n} \varepsilon_{n, p}$ são $\mathcal{C}$-equivalentes se existe um germe de difeomorfismo $H:\left(\mathbb{R}^{n} \times \mathbb{R}^{p}, 0\right) \longrightarrow\left(\mathbb{R}^{n} \times \mathbb{R}^{p}, 0\right)$ e um germe de função suave $\theta$ : $\left(\mathbb{R}^{n} \times \mathbb{R}^{p}, 0\right) \longrightarrow\left(\mathbb{R}^{p}, 0\right)$ tais que $H(x, y)=(x, \theta(x, y)), \theta(x, 0)=0, \forall x \in\left(\mathbb{R}^{n}, 0\right) \mathrm{e}$ $H(x, f(x))=(x, g(x))$.

Definição 1.59: Dizemos que $f, g \in \varepsilon_{n, p}$ são $\mathcal{K}$-equivalentes se existem germes de difeomorfismos $H:\left(\mathbb{R}^{n} \times \mathbb{R}^{p}, 0\right) \longrightarrow\left(\mathbb{R}^{n} \times \mathbb{R}^{p}, 0\right)$ e $h:\left(\mathbb{R}^{n}, 0\right) \longrightarrow\left(\mathbb{R}^{n}, 0\right)$ e um germe de função suave $\theta:\left(\mathbb{R}^{n} \times \mathbb{R}^{p}, 0\right) \longrightarrow\left(\mathbb{R}^{p}, 0\right)$ tais que $H(x, y)=(h(x), \theta(x, y))$, $\theta(x, 0)=0, \forall x \in\left(\mathbb{R}^{n}, 0\right)$ e $H(x, f(x))=(h(x), g(h(x)))$.

Definição 1.60: Seja $f \in \varepsilon_{n, p}$, definimos $I(f)$ como o ideal gerado pelas funções componentes de $f$, isto é, se $f=\left(f_{1}, \ldots, f_{n}\right)$ então $I(f)=\left\langle f_{1}, \ldots, f_{p}\right\rangle \subset \varepsilon_{n}$.

Teorema 1.61: Sejam $f, g \in \mathscr{M}_{n} \varepsilon_{n, p}$, então são equivalentes:

1. $f, g$ são $\mathcal{C}$-equivalentes.

2. $I(f)=I(g)$.

3. Existe uma matriz invertivel $U=\left(\mu_{i j}\right)_{1 \leqslant i, j \leqslant p}, \mu_{i, j} \in \varepsilon_{n}$, tal que $f=U g$. 
Proposição 1.62: Sejam $f, g \in \mathscr{M}_{n} \varepsilon_{n, p}$, então $f$ e $g$ são $\mathcal{K}$-equivalentes se e somente se existe um germe de difeomorfismo $h:\left(\mathbb{R}^{n}, 0\right) \longrightarrow\left(\mathbb{R}^{n}, 0\right)$ tal que $f \circ h$ e g são $\mathcal{C}$ equivalentes. Logo $f$ e $g$ são $\mathcal{K}$-equivalentes se e somente se existe um germe de difeomorfismo $h:\left(\mathbb{R}^{n}, 0\right) \longrightarrow\left(\mathbb{R}^{n}, 0\right)$ tal que $h^{*}(I(f))=I(g)$.

Segue do Teorema 1.61 e da Proposição 1.62 o seguinte Corolario.

Corolário 1.63: Sejam $f, g \in \mathscr{M}_{n} \varepsilon_{n, p}$, uma condição suficiente e necessaria para que $f$ e g sejam $\mathcal{K}$-equivalentes é que exista uma matriz $M=\left(\mu_{i j}\right)_{1 \leqslant i, j \leqslant p}, \mu_{i j} \in \varepsilon_{n} \forall 1 \leqslant i, j \leqslant p$ e um germe de difeomorfismo $h:\left(\mathbb{R}^{n}, 0\right) \longrightarrow\left(\mathbb{R}^{n}, 0\right)$ tais que $g=M . f(h)$.

\subsection{Variedades Algébricas}

Definição 1.64: Um subconjunto $N \subset \mathbb{R}^{m}$ é chamado de variedade algébrica, se este é o conjunto de soluções de um sistema de equações polinomiais sobre $\mathbb{R}$.

Exemplo 1.65: Os seguintes conjuntos são variedades algébricas:

1. $A_{k}=\left\{x \in \mathbb{R}^{m}: x_{1}^{k+1}-x_{2}^{2}=x_{\geqslant 3}=0\right\}, k \geqslant 1$;

2. $D_{k}=\left\{x \in \mathbb{R}^{m}: x_{1}^{2} x_{2}-x_{2}^{k-1}=x_{\geqslant 3}=0\right\}, k \geqslant 4$

3. $E_{6}=\left\{x \in \mathbb{R}^{m}: x_{1}^{3}-x_{2}^{4}=x_{\geqslant 3}=0\right\}$;

4. $E_{7}=\left\{x \in \mathbb{R}^{m}: x_{1}^{3}-x_{1} x_{2}^{3}=x_{\geqslant 3}=0\right\}$;

5. $E_{8}=\left\{x \in \mathbb{R}^{m}: x_{1}^{3}-x_{2}^{5}=x_{\geqslant 3}=0\right\}$.

\subsection{Problema A}

No que segue trabalharemos com germes de funções, germes de formas diferenciáveis e germes de variedades tendo como fonte e meta a origem.

Na definição 1.25 vimos o que é um simplectomorfismo, mas agora nós consideramos aqueles que tem a seguinte propriedade: $\phi:\left(\mathbb{R}^{2 n}, 0\right) \longrightarrow\left(\mathbb{R}^{2 n}, 0\right)$ um germe de difeomorfismo do espaço simplético $\left(\mathbb{R}^{2 n}, \omega\right)$ tal que $\phi^{*}(\omega)=\omega$. No que segue sempre que dizemos simplectoformismo, estaremos falando de simplectomorfismo da definição 1.25 junto com a propriedade acima.

Definição 1.66: Sejam $N_{1}, N_{2} \subset \mathbb{R}^{2 n}$ subconjuntos que contém a origem. Estes são chamados de simplectomorfos se existe um simplectomorfismo $\phi$ tal que $\phi\left(N_{1}, 0\right)=\left(N_{2}, 0\right)$ 
Teorema 1.67 (Darboux-Givental ver $[\mathrm{A}-\mathrm{G}]$ ): $\quad$ 1. Sejam $N \subset \mathbb{R}^{2 n}$ uma subvariedade e $\omega_{0}, \omega_{1}$ duas formas simpléticas em $\mathbb{R}^{2 n}$ com a mesma restrição a $T N$. Então existe um germe de difeomorfismo $\Phi:\left(\mathbb{R}^{2 n}, 0\right) \longrightarrow\left(\mathbb{R}^{2 n}, 0\right)$ tal que $\Phi(x)=x$ para todo $x \in N$ e $\Phi^{*} \omega_{0}=\omega_{1}$.

2. Duas subvariedades $N_{1}, N_{2}$ do espaço simplético $\left(\mathbb{R}^{2 n}, \omega\right)$ de igual dimensão são simplectomorfos se e somente se as restrições $\left.\omega\right|_{T N_{1}},\left.\omega\right|_{T N_{2}}$ são difeomorfas.

Definição 1.68: Seja $\omega$ uma 2-forma numa variedade suave $M^{n} \subset \mathbb{R}^{m}$, então existe associada uma matriz quadrada com coeficientes reais. O posto da matriz na origem é chamado de posto de $\omega$.

Definição 1.69: Definimos $\operatorname{Symp}\left(\mathbb{R}^{2 n}\right)$ o conjunto das 2-formas simpléticas em $\mathbb{R}^{2 n}$ e $\left.\operatorname{Symp}\left(\mathbb{R}^{2 n}\right)\right|_{\mathbb{R}^{r}}=\left\{\left.\omega\right|_{T R^{r}} / \omega \in \operatorname{Symp}\left(\mathbb{R}^{2 n}\right)\right\}$.

Teorema 1.70 (ver $[\mathrm{A}-\mathrm{G}]):\left.\quad \operatorname{Symp}\left(\mathbb{R}^{2 n}\right)\right|_{\mathbb{R}^{r}}$ consiste das 2-formas fechadas em $\mathbb{R}^{r}$ de posto maior ou igual que $2(r-n)$.

O presente trabalho é dedicado a resolver o seguinte problema.

Problema A: Classificar com respeito ao grupo dos simplectomorfismos a classe de todas as variedades algébricas no espaço simplético $\left(\mathbb{R}^{2 n}, \omega\right)$ que são difeomorfas a uma variedade algébrica fixa $N$, onde $N$ é

1. $A_{k}=\left\{x \in \mathbb{R}^{2 n}: x_{1}^{k+1}-x_{2}^{2}=x_{\geqslant 3}=0\right\}, k \geqslant 1$;

2. $D_{k}=\left\{x \in \mathbb{R}^{2 n}: x_{1}^{2} x_{2}-x_{2}^{k-1}=x_{\geqslant 3}=0\right\}, k \geqslant 4$;

3. $E_{6}=\left\{x \in \mathbb{R}^{2 n}: x_{1}^{3}-x_{2}^{4}=x_{\geqslant 3}=0\right\}$;

4. $E_{7}=\left\{x \in \mathbb{R}^{2 n}: x_{1}^{3}-x_{1} x_{2}^{3}=x_{\geqslant 3}=0\right\}$;

5. $E_{8}=\left\{x \in \mathbb{R}^{2 n}: x_{1}^{3}-x_{2}^{5}=x_{\geqslant 3}=0\right\}$.

Exemplo 1.71 (Exemplo de simplectomorfismos): Considere $n \geqslant 2$, seja $N$ fixo qualquer dos conjuntos $A_{k}, D_{k}, E_{6}, E_{7}, E_{8}$ acima e considere $\omega=d x_{1} \wedge d y_{1}+d x_{2} \wedge d y_{2}+\ldots+d x_{n} \wedge d y_{n}$ a forma simplética em $\mathbb{R}^{2 n}$. Sejam $\phi, \rho$ funções suaves reais definidas num invertalo aberto $I \subset \mathbb{R}$, que levam o zero no zero. Considere

$$
\Phi\left(x_{1}, \ldots y_{n}\right)=\left(x_{1}+\phi\left(y_{1}\right), x_{2}+\rho\left(y_{2}\right), x_{3}, \ldots, y_{n}\right)
$$


então $\Phi$ é um simplectomorfismo. De fato

$$
\begin{aligned}
\Phi^{*}(\omega) & =d\left(x_{1}+\phi\left(y_{1}\right)\right) \wedge d y_{1}+d\left(x_{2}+\rho\left(y_{2}\right)\right) \wedge d y_{2}+\ldots+d x_{n} \wedge d y_{n} \\
& =\left(d x_{1}+\phi^{\prime}\left(y_{1}\right) d y_{1}\right) \wedge d y_{1}+\left(d x_{2}+\rho^{\prime}\left(y_{2}\right) d y_{2}\right) \wedge d y_{2}+\ldots+d x_{n} \wedge d y_{n} \\
& =d x_{1} \wedge d y_{1}+\phi^{\prime}\left(y_{1}\right) d y_{1} \wedge d y_{1}+d x_{2} \wedge d y_{2}+\rho^{\prime}\left(y_{2}\right) d y_{2} \wedge d y_{2}+\ldots+d x_{n} \wedge d y_{n} \\
& =d x_{1} \wedge d y_{1}+d x_{2} \wedge d y_{2}+\ldots+d x_{n} \wedge d y_{n} \\
& =\omega
\end{aligned}
$$

Por outro lado se $p=\left(a_{1}, \ldots, a_{n}, b_{1}, \ldots, b_{n}\right) \in N$, então $H\left(a_{1}, a_{2}\right)=0$ e $a_{3}=\ldots=$ $a_{n}=b_{1} \ldots=b_{n}=0$ (no caso $\left.n=2, b_{1}=b_{2}=0\right)$. Logo

$$
\begin{aligned}
\Phi(p) & =\left(a_{1}+\phi\left(b_{1}\right), a_{2}+\rho\left(b_{2}\right), a_{3}, \ldots, b_{n}\right) \\
& =p
\end{aligned}
$$

pois $\phi(0)=\rho(0)=0$. 


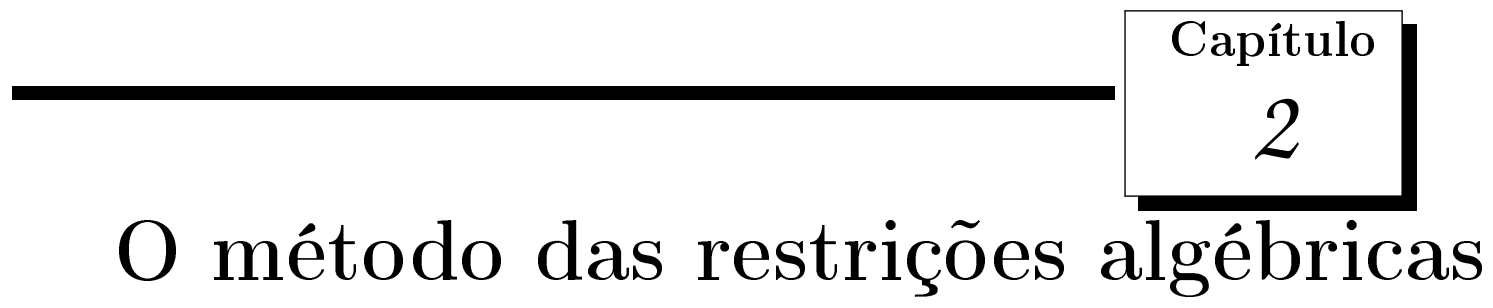

Este trabalho esta baseado do seguinte artigo [D-J-Z].

Neste capítulo definimos o espaço vetorial das restrições algébricas (é uma generalização da definição dada em [Z] de restrições algébricas de 1-formas), a ação do grupo de simetrias locais neste espaço vetorial, revisamos um resultado do grupo de cohomologia, estudamos alguns teoremas e além disso resolveremos o Problema A para o caso $N=\left\{A_{k}=0\right\}, k \in \mathbb{N}$. No caso geral de $N$, reduzimos o Problema A ao Problema B.

No que segue omitiremos as palavras suave e diferenciável, lembramos também que trabalhamos com germes de funções e de variedades tendo como fonte e meta a origem. Além disso $p$ sempre denotara um número natural.

\subsection{Definição de restrição algébrica}

Dado um germe de uma variedade $(M, 0)$ denote por $\Lambda^{p}(M)$ o $\mathbb{R}$-espaço vetorial de todos os germes em $x=0$, das $p$-formas diferenciáveis em $M$ (aqui consideramos as $p$ formas a diferença das $k$-formas na subseção 1.1.2). Dado um subconjunto $N \subset M$ tal que $0 \in N$, definimos os seguintes $\mathbb{R}$-subespaços vetorias de $\Lambda^{p}(M)$

$$
\begin{aligned}
& \Lambda_{N}^{p}(M)=\left\{\omega \in \Lambda^{p}(M): \omega(x)=0, \forall x \in N\right\} \\
& \mathscr{A}_{0}^{p}(N, M)=\left\{\alpha+d \beta: \alpha \in \Lambda_{N}^{p}(M), \beta \in \Lambda_{N}^{p-1}(M)\right\} .
\end{aligned}
$$

Observação 2.1: Seja $\omega \in \Lambda_{N}^{p}(M)$, lembre que $\omega(x)=0$ significa $\omega(x)\left(v_{1}, v_{2}, \ldots, v_{p}\right)=$ $0, \forall x \in(N, 0), \forall v_{i} \in T_{x} M, i=1, \ldots, p$. Seja $m=\operatorname{dim} M$, dado $\left(U, x_{1}, \ldots, x_{m}\right)$ um sistema de coordenadas local de $M$ em $x_{0} \in N$ existem funções $f_{i_{1}, i_{2}, \ldots, i_{p}}: U \rightarrow \mathbb{R}$, com $1 \leqslant i_{1}<\ldots<i_{p} \leqslant m$ tais que

$$
\omega(x)=\sum_{1 \leqslant i_{1}<\ldots<i_{p} \leqslant 2 n} f_{i_{1}, i_{2}, \ldots, i_{p}}(x) d x_{i_{1}} \wedge d x_{i_{2}} \wedge \ldots \wedge d x_{i_{p}} \text {, para todo } x \in U .
$$


Agora de $\omega\left(x_{0}\right)=0$ segue que $f_{i_{1}, i_{2}, \ldots, i_{p}}\left(x_{0}\right)=0$. Portanto temos que os coeficientes de $\omega$ se anulam em $x \in(N, 0)$.

Vejamos o que acontece no caso em que $(N, 0) \subset(M, 0)$ é um subvariedade, onde $(\mathrm{M}, 0)$ é uma subvariedade de $\mathbb{R}^{k}$.

Proposição 2.2: Se $N$ é uma subvariedade de $M$ então uma p-forma $\omega$ em $M$ satisfaz:

$$
\left.\omega\right|_{T N}=0 \Longleftrightarrow \omega \in \mathscr{A}_{0}^{p}(N, M)
$$

Demonstração: Suponha $\operatorname{dim} M=m$ e $\operatorname{dim} N=n$. Temos que existe $(U, \phi=$ $\left.\left(x_{1}, \ldots, x_{m}\right)\right)$ sistema de coordenadas local de $M$ em $x=0$ tal que $\phi: U \cap N \longrightarrow$ $V \subset \mathbb{R}^{n} \times\{0\}$, isto é, $\phi(x)=\left(x_{1}(x), x_{2}(x), \ldots, x_{n}(x), 0, \ldots, 0\right)$ para todo $x \in N \cap U$ e $\omega$ é como na Equação (2.1).

Suponha que $\left.\omega\right|_{T N}=0$.

Então

$$
\begin{aligned}
\omega(x) & =\sum_{1 \leqslant i_{1}<\ldots<i_{p} \leqslant m} f_{i_{1}, i_{2}, \ldots, i_{p}}(x) d x_{i_{1}} \wedge d x_{i_{2}} \wedge \ldots \wedge d x_{i_{p}} \\
& =\sum_{1 \leqslant i_{1}<\ldots<i_{p} \leqslant m} f_{i_{1}, i_{2}, \ldots, i_{p}}(x) \underbrace{d x_{i_{1}} \wedge d x_{i_{2}} \wedge \ldots \wedge d x_{i_{p}}}_{\text {não aparece } d x_{k}, n+1 \leqslant k \leqslant m}+\sum_{i=n+1}^{m} d x_{i} \wedge \underbrace{\mu_{i}}_{(p-1) \text {-forma }}
\end{aligned}
$$

segue do Lema de Hadamard aplicado a cada $f_{i_{1}, i_{2}, \ldots, i_{p}}$ na origem $\left(f_{i_{1}, i_{2}, \ldots, i_{p}}(0)=0\right)$ que $\omega=\sum_{i=n+1}^{m} x_{i} \alpha_{i}+\sum_{i=n+1}^{m} d x_{i} \wedge \mu_{i}$, onde as $\alpha_{i}$ são $p$-formas. Usando o fato $d\left(x_{i} \mu_{i}\right)=d x_{i} \wedge$ $\mu_{i}+x_{i} d \mu_{i}$, temos $\omega=\sum x_{i}\left(\alpha_{i}-d \mu_{i}\right)+d\left(\sum x_{i} \mu_{i}\right)$. Portanto $\omega \in A_{0}^{p}(N, M)$.

Reciprocamente, suponha que $\omega \in \mathscr{A}_{0}^{p}(N, M)$, então existem $\Lambda_{N}^{p}(M), \beta \in \Lambda_{N}^{p-1}(M)$ tais que $\omega=\alpha+d \beta$, logo pelo Lema de Hadamard em $\alpha$ e $\beta$ temos

$$
\begin{aligned}
\omega & =\sum_{i=n+1}^{m} x_{i} \alpha_{i}+d\left(\sum_{i=n+1}^{m} x_{i} \mu_{i}\right) \\
& =\sum_{i=n+1}^{m} x_{i}\left(\alpha_{i}+d \mu_{i}\right)+\sum_{i=n+1}^{m} d x_{i} \wedge \mu_{i}
\end{aligned}
$$

e portanto obtemos $\left.\omega\right|_{T N}=0$.

Agora dada uma subvariedade $M^{m}$ de $\mathbb{R}^{l}$ definimos uma relação de equivalência no espaço $\Lambda^{p}(M)$ para um subconjunto $(N, 0)$ qualquer de $(M, 0)$.

Definição 2.3 (Restrição algébrica): Seja $M^{m} \subset \mathbb{R}^{l}$ uma subvariedade, $N$ um subconjunto de $M$ e $\omega, \tilde{\omega} \in \Lambda^{p}(M)$. Definimos a relação de equivalência " $\sim_{N}$ " em $\Lambda^{p}(M)$ 
por:

$$
\omega \sim_{N} \tilde{\omega} \Leftrightarrow \omega-\tilde{\omega} \in \mathscr{A}_{0}^{p}(N, M)
$$

Denotamos por $\left[\Lambda^{p}(M)\right]_{N}$ o conjunto de classes de equivalência. A órbita de $\omega \in \Lambda^{p}(M)$ é chamada de restrição algébrica de $\omega$ a $N$ (ou também restrição algébrica a $N$ de $\omega$ ) e é denotada por $[\omega]_{N}$. Note que $0=[0]_{N}=\mathscr{A}_{0}^{p}(N, M)$.

Note também que o $\mathbb{R}$-espaço vetorial $\mathscr{A}_{0}^{p}(N, M)$ induz uma estrutura de $\mathbb{R}$-espaço vetorial natural em $\left[\Lambda^{p}(M)\right]_{N}$.

Proposição 2.4: Sejam $(M, 0) \subset \mathbb{R}^{l}$ uma subvariedade, $(N, 0)$ um subconjunto de $(M, 0)$, w uma p-forma $(p \in \mathbb{N})$, $\mu$ uma q-forma $\left(q \in \mathbb{N}_{0}\right)$ em $(M, 0)$, então temos

1. Se $\omega \in \mathscr{A}_{0}^{p}(N, M)$ então $d \omega \in \mathscr{A}_{0}^{p+1}(N, M)$.

2. Se $\omega \in \mathscr{A}_{0}^{p}(N, M)$ então $\omega \wedge \mu \in \mathscr{A}_{0}^{p+q}(N, M)$.

\section{Demonstração:}

1. Segue do fato $d^{2}=0$.

2. Da hipótese segue que $\omega=\alpha+d \beta \operatorname{com} \alpha \in \Lambda_{N}^{p}(M), \beta \in \Lambda_{N}^{p-1}(M)$.

Se $q \geqslant 1, \omega \wedge \mu=\alpha \wedge \mu+d \beta \wedge \mu$ e como $d \beta \wedge \mu=d(\beta \wedge \mu)+(-1)^{q} \beta \wedge d \mu$ obtemos

$$
\omega \wedge \mu=\alpha \wedge \mu+(-1)^{q} \beta \wedge d \mu+d(\beta \wedge \mu) \in \mathscr{A}_{0}^{p+q}(N, M)
$$

pois $\quad \alpha \wedge \mu+(-1)^{q} \beta \wedge d \mu \in \Lambda_{N}^{p+q}(M), \quad \beta \wedge \mu \in \Lambda_{N}^{p+q-1}(M)$.

Se $q=0$, segue de $d(\beta \mu)=(d \beta) \mu+\beta \wedge d \mu$.

A Proposição 2.4 garante que as seguintes aplicações estão bem definidas.

Definição 2.5: Seja $(M, 0) \subset \mathbb{R}^{l}$ uma subvariedade, $(N, 0) \subset(M, 0)$ um subconjunto, $q \in \mathbb{N}_{0}, p \in \mathbb{N}$. Então temos

$$
\begin{aligned}
& {\left[\Lambda^{p}(M)\right]_{N} \longrightarrow\left[\Lambda^{(p+1)}(M)\right]_{N} \quad\left[\Lambda^{p}(M)\right]_{N} \times\left[\Lambda^{q}(M)\right]_{N} \longrightarrow\left[\Lambda^{(p+q)}(M)\right]_{N}} \\
& {[\omega]_{N} \quad \longmapsto \quad[d \omega]_{N} \quad\left([\omega]_{N},[\alpha]_{N}\right) \quad \longmapsto \quad[\omega \wedge \alpha]_{N}}
\end{aligned}
$$

Denotamos estas operações por $d$ e $\wedge$ respectivamente. Assim temos $d[\omega]_{N}=[d \omega]_{N}$ e $[\omega]_{N} \wedge[\alpha]_{N}=[\omega \wedge \alpha]_{N}$.

A Proposição 2.4 também garante que no caso $q=0$ temos que $\left[\Lambda^{p}(M)\right]_{N}$ tem uma estrutura de $\Lambda^{0}(M)$-módulo onde a operação e dada por $\left(f,[\theta]_{N}\right) \mapsto[f . \theta]_{N}$. Denotamos por $\left\langle[\theta]_{N}\right\rangle$ o $\Lambda^{0}(M)$-submódulo gerado por $[\theta]_{N} \in\left[\Lambda^{p}(M)\right]_{N}$. 
Exemplo 2.6: (Restrições algébricas de 2-formas a $A_{k}$ ) Neste exemplo obtemos uma base para o espaço vetorial $\left[\Lambda^{2}\left(\mathbb{R}^{2 n}\right)\right]_{N}$, onde $N=A_{k}=\left\{x \in \mathbb{R}^{2 n}: x_{1}^{k+1}-x_{2}^{2}=x_{\geqslant 3}=0\right\}$ e $k \geqslant 1$.

Seja $H(x)=x_{1}^{k+1}-x_{2}^{2}$, por definição segue que

$$
[d H]_{N}=\left[d x_{\geqslant 3}\right]_{N}=0
$$

logo pela Proposição 2.4

$$
\left[d H \wedge d x_{1}\right]_{N}=\left[d H \wedge d x_{2}\right]_{N}=0
$$

isto é,

$$
\left[\frac{\partial H}{\partial x_{2}} d x_{1} \wedge d x_{2}\right]_{N}=\left[\frac{\partial H}{\partial x_{1}} d x_{1} \wedge d x_{2}\right]_{N}=0
$$

Seja $\omega$ uma 2-forma, então por (2.2) temos $[\omega]_{N}=\left[f\left(x_{1}, x_{2}\right) d x_{1} \wedge d x_{2}\right]_{N}$. Da equação (2.3) segue como caso particular: se $f \in\langle\nabla H\rangle=\left\langle x_{1}{ }^{k}, x_{2}\right\rangle$ então $[\omega]_{N}=0$. No caso geral, temos que existem funções $f_{i}, i=0, \ldots, k$, definidas numa vizinhança da origem tais que

$$
f\left(x_{1}, x_{2}\right)=f(0)+j^{k-1} f(0)+\sum_{i=0}^{k} x_{1}^{i} x_{2}^{k-i} f_{i}\left(x_{1}, x_{2}\right)
$$

logo a Proposição 2.4 e o caso particular anterior implicam $\left[\sum_{i=0}^{k} x_{1}{ }^{i} x_{2}{ }^{k-i} f_{i} d x_{1} \wedge d x_{2}\right]_{N}=$ 0 e $\left[j^{k-1} f(0) d x_{1} \wedge d x_{2}\right]_{N}=\left[\sum_{i=1}^{k-1} c_{i} x_{1}^{i} d x_{1} \wedge d x_{2}\right]_{N}$ para alguns $c_{i} \in \mathbb{R}, i=1, \ldots, k-1$. Portanto

$$
[\omega]_{N}=\left[\sum_{i=0}^{k-1} c_{i} x_{1}^{i} d x_{1} \wedge d x_{2}\right]_{N} .
$$

No Capítulo 4 mostraremos que $\left[\sum_{i=0}^{k-1} c_{i} x_{1}{ }^{i} d x_{1} \wedge d x_{2}\right]_{N}=0$ se e somente se $\sum_{i=0}^{k-1} x_{1}{ }^{i} \in$ $\left\langle x_{1}{ }^{k}, x_{2}\right\rangle$, logo comparando os $k$-jatos temos $c_{i}=0, i=0, \ldots, k-1$. Portanto $\left\{\left[x_{1}{ }^{i} d x_{1} \wedge\right.\right.$ $\left.\left.d x_{2}\right]_{N}\right\}_{i=0}^{k-1}$ é uma base do espaço vetorial $\left[\Lambda^{2}\left(\mathbb{R}^{2 n}\right)\right]_{N}$.

Logo a dimensão do espaço das restrições algébricas em $A_{k}$ de todas as 2-formas em $\mathbb{R}^{2 n}$ é igual a $k$ e este espaço é gerado pelas restrições denotadas por

$$
\left[A_{k}\right]^{i}=\left[x_{1}^{i} d x_{1} \wedge d x_{2}\right]_{A_{k}} .
$$

\subsection{A ação do grupo das simetrias locais}

Nesta seção dado um germe de subvariedade $(M, 0)$ de $\mathbb{R}^{n}$ introduzimos uma ação de um subgrupo do grupo de germes de difeomorfismos de $M, D(M)=\{h:(M, 0) \rightarrow$ $(M, 0) / h$ é germe de difeomorfismo $\}$ no espaço $\left[\Lambda^{p}(M)\right]_{N}$. 
Proposição 2.7: Sejam $M \subset \mathbb{R}^{n}$ e $\tilde{M} \subset \mathbb{R}^{m}$ variedades de mesma dimensão, e $0 \in M$ e $0 \in \tilde{M}$. Seja $\Phi:(\tilde{M}, 0) \longrightarrow(M, 0)$ um germe de difeomorfismo e $N \subset M$ suconjunto tal que $0 \in N$. Então $\Phi^{*}\left(\mathscr{A}_{0}^{p}(N, M)\right)=\mathscr{A}_{0}^{p}\left(\Phi^{-1}(N), \tilde{M}\right)$.

Demonstração: Considere $\omega \in \mathscr{A}_{0}^{p}(N, M)$, então existem $\alpha \in \Lambda_{N}^{p}(M)$ e $\beta \in \Lambda_{N}^{p-1}(M)$ tais que $\omega=\alpha+d \beta$. Logo

$$
\Phi^{*} \omega=\Phi^{*} \alpha+\Phi^{*} d \beta=\Phi^{*} \alpha+d\left(\Phi^{*} \beta\right)
$$

onde $\Phi^{*} \alpha \in \Lambda_{\Phi^{-1}(N)}^{p}(\tilde{M})$ e $\Phi^{*} \beta \in \Lambda_{\Phi^{-1}(N)}^{p-1}(\tilde{M})$.

Agora suponha $\tilde{\omega} \in \mathscr{A}_{0}^{p}\left(\Phi^{-1}(N), \tilde{M}\right)$, então existem $\tilde{\alpha} \in \Lambda_{N}^{p}(\tilde{M})$ e $\tilde{\beta} \in \Lambda_{N}^{p-1}(\tilde{M})$ tais que $\tilde{\omega}=\tilde{\alpha}+d \tilde{\beta}$. Logo existe $U$ uma vizinhança de $x=0$ tal que $\left.\tilde{\alpha}\right|_{\Phi^{-1}(N) \cap U}=$ $\left.\tilde{\beta}\right|_{\Phi^{-1}(N) \cap U}=0$. Podemos escolher $U$ de modo que existe $V$, uma vizinhaça de $y=0$, onde $\left.\Phi\right|_{U}: U \longrightarrow V$ é difeomorfismo.

Seja $\omega:=\left(\left(\left.\Phi\right|_{U}\right)^{-1}\right)^{*}(\tilde{\omega})$, então temos $\Phi^{*} \omega=\Phi^{*}\left(\left(\left.\Phi\right|_{U}\right)^{-1}\right)^{*}(\tilde{\omega})=\tilde{\omega}$.

Definição 2.8: Seja $(\tilde{N}, 0) \subset(\tilde{M}, 0)$ e $(N, 0) \subset(M, 0)$. Duas restrições algébricas $[\omega]_{N} \in\left[\Lambda^{p}(M)\right]_{N},[\tilde{\omega}]_{\tilde{N}} \in\left[\Lambda^{p}(\tilde{M})\right]_{\tilde{N}}$ são difeomorfas, se existe, um germe de difeomorfismo $\Phi:(\tilde{M}, 0) \longrightarrow(M, 0)$ tal que $\Phi(\tilde{N}, 0)=(N, 0)$ e $\Phi^{*}\left([\omega]_{N}\right)=[\tilde{\omega}]_{\tilde{N}}$.

No caso $\tilde{N}=N$ e $\tilde{M}=M$ a Proposição 2.7 garante que a seguinte aplicação está bem definida.

Definição 2.9: Sejam $N, M$ como na proposição anterior. Definimos a aplicação

$$
\begin{array}{rlc}
{ }^{*}: D_{N}(M) \times\left[\Lambda^{p}(M)\right]_{N} & \longrightarrow & {\left[\Lambda^{p}(M)\right]_{N}} \\
\left(\Phi,[\omega]_{N}\right) & \longmapsto & \Phi^{*}\left([\omega]_{N}\right)=\left[\Phi^{*} \omega\right]_{N}
\end{array}
$$

onde $D_{N}(M)$ denota o conjunto de germes de difeomorfismos de $M$ que preservan $N$, seus elementos são chamados de simtrias locais de $N$.

Observação 2.10: O conjunto de simetrias locais de $N$ é um subgrupo de $D(M)$ e a aplicação da Definição 2.9 é uma ação deste subgrupo no espaço $\left[\Lambda^{p}(M)\right]_{N}$.

\subsection{Teorema de Redução}

Agora suponha que $N \subset \mathbb{R}^{m}$ está contido numa subvariedade $M$ de $\mathbb{R}^{m}$. O seguinte resultado mostra que a classificação das restrições algébricas das $p$-formas em $\mathbb{R}^{m}$ a $N$ é equivalente à classificação das restrições algébricas das $p$-formas em $M$ a $N$. 
Proposição 2.11: $\operatorname{Seja}(M, 0) \subset \mathbb{R}^{m}$ um germe de variedade e $(N, 0) \subset(M, 0)$ subconjunto, sejam $\omega_{1}, \omega_{2}$ p-formas em $\mathbb{R}^{m}$. Ent $\tilde{a} o\left[\omega_{1}\right]_{N}=\left[\omega_{2}\right]_{N} \Longleftrightarrow\left[\left.\omega_{1}\right|_{T M}\right]_{N}=\left[\left.\omega_{2}\right|_{T M}\right]_{N}$.

Demonstração: Provaremos somente uma implicação.

Suponha que $\left[\omega_{1}\right]_{N}=\left[\omega_{2}\right]_{N}$. Isto significa que existem $\alpha \in \Lambda_{N}^{p}\left(\mathbb{R}^{m}\right)$ e $\beta \in \Lambda_{N}^{p-1}\left(\mathbb{R}^{m}\right)$ tais que $\omega_{1}-\omega_{2}=\alpha+d \beta$. Restringindo a $T M$ temos $\left.\left(\omega_{1}-\omega_{2}\right)\right|_{T M}=\left.\alpha\right|_{T M}+\left.(d \beta)\right|_{T M}=\left.\alpha\right|_{T M}+$ $d\left(\left.\beta\right|_{T M}\right)$. Note que $\left.\alpha\right|_{T M} \in \Lambda_{N}^{p}(M)$ e $\left.\beta\right|_{T M} \in \Lambda_{N}^{p-1}(M)$, portanto $\left[\left.\omega_{1}\right|_{T M}\right]_{N}=\left[\left.\omega_{2}\right|_{T M}\right]_{N}$.

O seguinte Teorema generaliza a Proposição 2.11.

Teorema 2.12: Sejam $\left(N_{1}, 0\right)$ e $\left(N_{2}, 0\right)$ subconjuntos de $\left(\mathbb{R}^{m}, 0\right)$ contidos nas subvariedades de mesma dimensão $\left(M_{1}, 0\right)$ e $\left(M_{2}, 0\right)$ respectivamente. Sejam $\omega_{1}$ e $\omega_{2}$ germes de p-formas em $\mathbb{R}^{m}$. As restrições algébricas $\left[\omega_{1}\right]_{N_{1}}$ e $\left[\omega_{2}\right]_{N_{2}}$ são difeomorfas se e só se as restrições algébricas $\left[\left.\omega_{1}\right|_{T M_{1}}\right]_{N_{1}}$ e $\left[\left.\omega_{2}\right|_{T M_{2}}\right]_{N_{2}}$ são difeomorfas.

Exemplo 2.13: (Classificação das restrições algébricas de 2-formas a $A_{k}$ )

A curva $A_{k}$ tem uma simetria local da forma $\Phi\left(x_{1}, x_{2}\right)=\left(x_{1} \phi^{2}, x_{2} \phi^{k+1}\right)$ onde $\phi=$ $\phi\left(x_{1}, x_{2}\right)$ e $\phi(0,0)=1$. Considere as simetrias locais

$$
\Phi_{r, s}\left(x_{1}, x_{2}\right)=\left(x_{1}\left(1+r x_{1}^{s}\right)^{2}, x_{2}\left(1+r x_{1}^{s}\right)^{k+1}\right) \text { onde } r, s \in \mathbb{R}, s \geqslant 1 \text {. }
$$

Considere $a \in \mathbb{R}$ então temos

$$
\begin{aligned}
\Phi_{r, s}^{*}\left[a x_{1}^{p} d x_{1} \wedge d x_{2}\right]_{A_{k}}= & {\left[a\left(x_{1}\left(1+r x_{1}^{s}\right)^{2}\right)^{p}\left(d\left(x_{1}\left(1+r x_{1}^{s}\right)^{2}\right)\right) \wedge d\left(x_{2}\left(1+r x_{1}^{s}\right)^{k+1}\right)\right]_{A_{k}} } \\
= & a\left[( x _ { 1 } ^ { p } ( 1 + r x _ { 1 } ^ { s } ) ^ { 2 p } ) \left\{\left(1+r x_{1}^{s}\right)^{2}+\right.\right. \\
& \left.\left.+2 s r x_{1}^{s}\left(1+r x_{1}^{s}\right)\right\}\left(1+r x_{1}^{s}\right)^{k+1} d x_{1} \wedge d x_{2}\right]_{A_{k}} \\
= & a\left[x_{1}^{p}\left(1+r x_{1}^{s}\right)^{2 p+k+2}\left(1+(2 s r+r) x_{1}^{s}\right) d x_{1} \wedge d x_{2}\right]_{A_{k}} \\
= & a\left[x_{1}^{p}\left\{\sum_{i=0}^{2 p+k+2}\left(\begin{array}{c}
2 p+k+2 \\
i
\end{array}\right)\left(r x_{1}^{s}\right)^{i}\right\}\left(1+(2 s r+r) x_{1}^{s}\right) d x_{1} \wedge d x_{2}\right]_{A_{k}} \\
= & a\left[\left(x_{1}^{p}+(2 p+k+2) r x_{1}^{s+p}+(2 s+1) r x_{1}^{s+p}+\right.\right. \\
& \left.\left.\quad+o\left\|\left(x_{1}\right)\right\|^{p+s}\right) d x_{1} \wedge d x_{2}\right]_{A_{k}} \\
& =a\left[\left(x_{1}^{p}+r(2 p+2 s+k+3) x_{1}^{s+p}+o\left\|\left(x_{1}\right)\right\|^{p+s}\right) d x_{1} \wedge d x_{2}\right]_{A_{k}}
\end{aligned}
$$

onde $o\left\|\left(x_{1}\right)\right\|^{p+s}$ representa a soma de monômios em $x_{1}$ de grau $>p+s$.

Logo se $\tilde{r}=r(2 p+2 s+k+3)$ segue que

$$
\Phi_{r, s}^{*}\left[a A_{k}\right]^{p} \in\left[a A_{k}\right]^{p}+\tilde{r}\left[a A_{k}\right]^{p+s}+\left\langle\left[A_{k}\right]^{p+s+1}, \ldots,\left[A_{k}\right]^{k-1}\right\rangle .
$$


Agora como $r$ e $s$ são quaisquer, segue que qualquer elemento do espaço afim $\left[A_{k}\right]^{p}+$ $\mathbb{R}\left\{\left[A_{k}\right]^{p+1}, \ldots,\left[A_{k}\right]^{k-1}\right\}$ é difeomorformo a $\left[A_{k}\right]^{p}$.

De fato, seja

$$
\left[A_{k}\right]^{p}+\sum_{i=1}^{k-1-p} \alpha_{i}\left[A_{k}\right]^{p+i} \in\left[A_{k}\right]^{p}+\mathbb{R}\left\{\left[A_{k}\right]^{p+1}, \ldots,\left[A_{k}\right]^{k-1}\right\} .
$$

Para provar que é difeomorfo a $\left[A_{k}\right]^{p}$ é suficiente aplicar $\Phi_{r, s}$ na equação (2.5) várias vezes com adequados $r$ e $s$. Por exemplo:

$$
\begin{aligned}
& s=1 \text { e } r_{1}=\frac{\alpha_{1}}{2 p+k+5} \text { temos } \\
& \Phi_{r_{1}, 1}{ }^{*}\left[A_{k}\right]^{p} \in\left[A_{k}\right]^{p}+\alpha_{1}\left[A_{k}\right]^{p+1}+b_{2}\left[A_{k}\right]^{p+2}+\mathbb{R}\left\{\left[A_{k}\right]^{p+3}, \ldots,\left[A_{k}\right]^{k-1}\right\} \\
& s=2 \text { e } r_{2}=\frac{\alpha_{2}-b_{2}}{2 p+k+7} \text { temos } \\
& \Phi_{r_{2}, 2}{ }^{*} \Phi_{r_{1}, 1}{ }^{*}\left[A_{k}\right]^{p} \in \Phi_{r_{2}, 2}{ }^{*}\left[A_{k}\right]^{p}+\Phi_{r_{2}, 2}{ }^{*}\left(\alpha_{1}\left[A_{k}\right]^{p+1}\right)+\Phi_{r_{2}, 2}{ }^{*}\left(b_{2}\left[A_{k}\right]^{p+2}\right)+ \\
& +\Phi_{r_{2}, 2} *\left(\mathbb{R}\left\{\left[A_{k}\right]^{p+2}, \ldots,\left[A_{k}\right]^{k-1}\right\},\right. \\
& \text { - } \Phi_{r_{2}, 2}{ }^{*}\left[A_{k}\right]^{p} \in\left[A_{k}\right]^{p}+\left(\alpha_{2}-b_{2}\right)\left[A_{k}\right]^{p+2}+\mathbb{R}\left\{\left[A_{k}\right]^{p+3}, \ldots,\left[A_{k}\right]^{k-1}\right\} \text {, } \\
& \text { - } \Phi_{r_{2}, 2}{ }^{*}\left(\alpha_{1}\left[A_{k}\right]^{p+1}\right) \in \alpha_{1}\left[A_{k}\right]^{p+1}+\mathbb{R}\left\{\left[A_{k}\right]^{p+3}, \ldots,\left[A_{k}\right]^{k-1}\right\} \text {, } \\
& \text { - } \Phi_{r_{2}, 2}{ }^{*}\left(b_{2}\left[A_{k}\right]^{p+2}\right) \in b_{2}\left[A_{k}\right]^{p+2}+\mathbb{R}\left\{\left[A_{k}\right]^{p+4}, \ldots,\left[A_{k}\right]^{k-1}\right\} \text {, } \\
& \text { - } \Phi_{r_{2}, 2} *\left(\mathbb{R}\left\{\left[A_{k}\right]^{p+3}, \ldots,\left[A_{k}\right]^{k-1}\right\}\right) \subset \mathbb{R}\left\{\left[A_{k}\right]^{p+3}, \ldots,\left[A_{k}\right]^{k-1}\right\},
\end{aligned}
$$

Portanto $\Phi_{r_{2}, 2}{ }^{*} \Phi_{r_{1}, 1}{ }^{*}\left[A_{k}\right]^{p} \in\left[A_{k}\right]^{p}+\alpha_{1}\left[A_{k}\right]^{p+1}+\alpha_{2}\left[A_{k}\right]^{p+2}+\mathbb{R}\left\{\left[A_{k}\right]^{p+3}, \ldots,\left[A_{k}\right]^{k-1}\right\}$. Continuando com este procedimento obtemos $\Phi=\Phi_{r_{1}, 1} \circ \Phi_{r_{2}, 2} \circ \ldots \circ \Phi_{r_{k-1-p}, k-1-p}$ tal que

$$
\Phi^{*}\left[A_{k}\right]^{p}=\left[A_{k}\right]^{p}+\sum_{i=1}^{k-1-p} \alpha_{i}\left[A_{k}\right]^{p+i} .
$$

Logo qualquer restrição algébrica $\sum_{i=p}^{k-1} \alpha_{i}\left[A_{k}\right]^{i}$ tal que $\alpha_{p} \neq 0$ é difeomorfa a $\alpha_{p}\left[A_{k}\right]^{p}$. Agora a restrição $\alpha_{p}\left[A_{k}\right]^{p}$ é difeormorfo a $\left[A_{k}\right]^{p}$, para isso usamos simetrias locais $\left(x_{1}, x_{2}\right) \longmapsto\left(t^{2} x_{1}, t^{k+1} x_{2}\right)$ e/ou $\left(x_{1}, x_{2}\right) \longmapsto\left(x_{1},-x_{2}\right)$.

Por último, no Capítulo 5 se mostra, que se as restrições $\left[A_{k}\right]^{i}$ são difeomorfas $\left[A_{k}\right]^{j}$ com $0 \leqslant i<j \leqslant k-1$, então os ideais $\left\langle x_{1}^{i}, x_{2}\right\rangle$ e $\left\langle x_{1}^{j}, x_{2}\right\rangle$ são isomorfos (este isomorfismo é induzido por um germe de difeomorfismo) o que é uma contradição (como será feito numa demonstração do Capítulo 5).

Assim obtemos:

Qualquer restrição algébrica de uma 2-forma em $\mathbb{R}^{m}$ a $A_{k}$ é difeomorfa a uma e só uma restrição algébrica $\left[x_{1}^{i} d x_{1} \wedge d x_{2}\right]_{A_{k}}, i=0,1, \ldots, k$. 


\subsection{Grupos de Cohomologia Relativa}

O nome "restrição algébrica" foi introduzida em [Z], mas tem uma relação entre $\mathscr{A}_{0}^{p}(N, M)$ e o grupo de cohomologia relativo

$$
H^{p}\left(N, \mathbb{R}^{m}\right)=\frac{\left\{\omega \in \mathscr{A}_{0}^{p}(N, M): d \omega=0\right\}}{\left\{d \alpha: \alpha \in \mathscr{A}_{0}^{p-1}\left(N, \mathbb{R}^{m}\right)\right\}} .
$$

Definição 2.14: Um conjunto $N \subset \mathbb{R}^{m}$ é chamado quase-homogêneo se existe um sistema de coordenadas local $x_{1}, x_{2}, \ldots, x_{m}$ e números positivos $\lambda_{1}, \ldots, \lambda_{m}$ tais que: se um ponto com coordenadas $x_{i}=a_{i}$ pertence a $N$ então para qualquer $t \in[0,1]$ o ponto com coordenadas $x_{i}=t^{\lambda_{i}} a_{i}$ tambem pertence a $N$, isto é,

$$
\left(a_{1}, a_{2}, \ldots, a_{m}\right) \in N \Rightarrow\left(t^{\lambda_{1}} a_{1}, t^{\lambda_{2}} a_{2}, \ldots, t^{\lambda_{n}} a_{n}\right) \in N, \forall t \in[0,1]
$$

Teorema 2.15 (ver $[\mathrm{R}]): S e N \subset \mathbb{R}^{m}$ é subconjunto quase-homogêneo então $H^{p}\left(N, \mathbb{R}^{m}\right)=$ $\{0\}$, para qualquer $p \geqslant 1$.

\subsection{Teoremas A e B}

Nesta seção estudamos os principais teoremas que serão usados em nossa classificação.

O método de restrições algébricas é baseado na seguinte generalização do Teorema 1.67 .

Teorema 2.16 (Teorema A): $\quad$ 1. Seja $(N, 0) \subset \mathbb{R}^{2 n}$ um subconjunto quase-homogêneo. Sejam $\omega_{0}, \omega_{1}$ formas simpléticas em $\mathbb{R}^{2 n}$ com a mesma restrição algébrica a $N$. Então existe um germe de difeomorfismo $\Phi:\left(\mathbb{R}^{2 n}, 0\right) \longrightarrow\left(\mathbb{R}^{2 n}, 0\right)$ tal que $\Phi(x)=x$ para todo $x \in(N, 0)$ e $\Phi^{*} \omega_{1}=\omega_{0}$.

2. Dois subconjuntos quase-homogêneos $N_{1}, N_{2}$ de um espaço simplético fixado $\left(\mathbb{R}^{2 n}, \omega\right)$ são simplectomorfos se e somente se as restrições algébricas da forma simplética $\omega$ a $N_{1}$ e $N_{2}$ são difeomorfas.

Observação 2.17: A prova mostrará que no Teorema A a hipótese de $N$ ser quasehomogênea pode ser substituída por $H^{2}\left(N, \mathbb{R}^{2 n}\right)=\{0\}$.

Antes de demonstrar o Teorema A vejamos uma aplicação, para isso fixamos as seguintes notações:

- $\left[\Lambda^{2 \text {,closed }}\left(\mathbb{R}^{2 n}\right)\right]_{N}$ : O subespaço vetorial de $\left[\Lambda^{2}\left(\mathbb{R}^{2 n}\right)\right]_{N}$ das restrições algébricas de todas as 2-formas fechadas em $\mathbb{R}^{2 n}$ a $N$. 
- $\left[\operatorname{Symp}\left(\mathbb{R}^{2 n}\right)\right]_{N}$ : O subconjunto aberto de $\left[\Lambda^{2, \text { closed }}\left(\mathbb{R}^{2 n}\right)\right]_{N}$ das restrições algébricas de formas simpléticas em $\mathbb{R}^{2 n}$ a $N$.

Considere o seguinte problema

Problema B: Classificar as restrições algébricas no conjunto $\left[\operatorname{Symp}\left(\mathbb{R}^{2 n}\right)\right]_{N}$ com respeito ao grupo de simetrias locais de $N$.

Proposição 2.18: Seja $N \subset \mathbb{R}^{2 n}$ um conjunto quase-homogêneo então resolver o Problema B implica resolver o Problema A.

Demonstração: De fato, suponha que o problema B é resolvido, assim temos uma lista de formas normais $\left[\theta_{1}\right]_{N}, \ldots,\left[\theta_{s}\right]_{N} \in\left[\operatorname{Symp}\left(\mathbb{R}^{2 n}\right)\right]_{N}$, de restrições algébricas, onde $\theta_{i}$ são 2 -formas $i=1, \ldots, s$. Logo existem formas simpléticas $\omega_{i}$ tais que $\left[\omega_{i}\right]_{N}=\left[\theta_{i}\right]_{N}, \forall i=$ $1, \ldots, s$.

Agora, dado um espaço simplético $\left(\mathbb{R}^{2 n}, \omega_{0}\right)$, tomamos os germes de difeomorfismos $\phi_{i}$ : $\left(\mathbb{R}^{2 n}, 0\right) \longrightarrow\left(\mathbb{R}^{2 n}, 0\right)$ tais que $\Phi_{i}^{*}\left(\omega_{i}\right)=\omega_{0}, i=1, \ldots, s$ (existem pelo Teorema Clássico de Darboux).

Considere $N^{i}=\Phi_{i}^{-1}(N), 1 \leqslant i \leqslant s$. Suponha que $N^{i}$ e $N^{j}(i \neq j)$ são simplectomofor$\operatorname{mos}, \operatorname{logo}$ existe $\phi:\left(\mathbb{R}^{2 n}, 0\right) \rightarrow\left(\mathbb{R}^{2 n}, 0\right)$ tal que $\phi^{*} \omega_{0}=\omega_{0}$ e $\phi\left(N^{j}\right) \subset N^{i}$, então temos que $\psi=\Phi_{i} \circ \phi \circ \Phi_{j}^{-1}$ é uma simetria local que leva $\left[\omega_{i}\right]_{N}$ em $\left[\omega_{j}\right]_{N}$ difeomorficamente, o que é uma contradição.

Agora suponha que $N_{0}$ é difeomorfo a $N$, isto é, existe $\psi:\left(\mathbb{R}^{2 n}, 0\right) \rightarrow\left(\mathbb{R}^{2 n}, 0\right)$ tal que $\psi(N)=N_{0}$.

Então temos que

$$
\psi^{*}\left(\left[\omega_{0}\right]_{N_{0}}\right)=\left[\psi^{*} \omega_{0}\right]_{N},
$$

logo pela hipótese existe $\phi:\left(\mathbb{R}^{2 n}, 0\right) \rightarrow\left(\mathbb{R}^{2 n}, 0\right)$ simetria local de $N$ tal que para algum $i$

$$
\phi^{*}\left[\psi^{*} \omega_{0}\right]_{N}=\left[\omega_{i}\right]_{N}
$$

O que implica $\phi^{*} \psi^{*}\left(\left[\omega_{0}\right]_{N_{0}}\right)=\left[\omega_{i}\right]_{N}$ consequentemente $\Phi_{i}^{*} \phi^{*} \psi^{*}\left(\left[\omega_{0}\right]_{N_{0}}\right)=\left[\omega_{0}\right]_{N^{i}}$, portanto $\left[\omega_{0}\right]_{N_{0}}$ e $\left[\omega_{0}\right]_{N^{i}}$ são difeomorfos e pelo Teorema A (2) $N_{0}$ e $N^{i}$ são simplectomorfos.

Logo $N^{1}, \ldots, N^{s}$ é uma lista final das formas normais do Problema A.

Como já foi mencionado para o caso $N=A_{k}$ o Problema A foi estudado por $\mathbf{V}$. Arnol'd em $[\mathrm{A}]$ para $k$ par. 
Note que para $n \geqslant 2$ temos

$$
A_{k} \subset \mathbb{R}^{2} \times\{0\}^{2 n-2}=M_{0} .
$$

Seja $\omega$ uma 2-forma, então $[\omega]_{A_{k}}=\left[\sum c_{i} x^{i} d x_{1} \wedge d x_{2}\right]_{A_{k}}$, logo pela Proposição 2.11 $\left[\left.\omega\right|_{T M_{0}}\right]_{A_{k}}=\left[\left.\left(\sum c_{i} x^{i} d x_{1} \wedge d x_{2}\right)\right|_{T M_{0}}\right]_{A_{k}}$ onde posto $\left(\sum c_{i} x^{i} d x_{1} \wedge d x_{2}\right) \geqslant 0 \geqslant 2(2-n)$ assim pelo Teorema 1.70 existe $\beta \in \operatorname{Symp}\left(\mathbb{R}^{2 n}\right)$ tal que $\left[\left.\omega\right|_{T M_{0}}\right]_{A_{k}}=\left[\left.\beta\right|_{T M_{0}}\right]_{A_{k}}$, que implica $[\omega]_{A_{k}}=[\beta]_{A_{k}}$.

Isto quer dizer que qualquer restrição algébrica de uma 2-forma em $\mathbb{R}^{2 n}$ a $A_{k}$ pode ser representada pela restrição algébrica de uma forma simplética, no caso $n \geqslant 2$.

Portanto os resultados dos Exemplos 2.6 e 2.13 mostram que na classificação do problema B com $N=A_{k} \subset \mathbb{R}^{2 n \geqslant 4}$ há exatamente $k+1$ órbitas

$$
\left[A_{k}\right]^{i}=\left[x_{1}^{i} d x_{1} \wedge d x_{2}\right]_{A_{k}}, i=0,1, \ldots, k .
$$

Note que para $i=1, \ldots, k, x_{1}^{i} d x_{1} \wedge d x_{2}$ não são 2-formas simpléticas, mas como $\left[A_{k}\right]^{i} \in$ $\left[\operatorname{Symp}\left(\mathbb{R}^{2 n}\right)\right]_{A_{k}}$, existe um representante simplético de $\left[A_{k}\right]^{i}$. Considere por exemplo as formas simpléticas

$$
\begin{aligned}
& \theta^{k}=d x_{1} \wedge d x_{3}+d x_{2} \wedge d x_{4}+d x_{5} \wedge d x_{6}+\ldots+d x_{2 n-1} \wedge d x_{2 n} \\
& \theta^{i}=x_{1}^{i} d x_{1} \wedge d x_{2}+\theta^{k}, 0 \leqslant i \leqslant k-1
\end{aligned}
$$

Então $\left[A_{k}\right]^{i}=\left[\theta^{i}\right]_{A_{k}}, \forall i=1, \ldots, k$.

Dada uma forma simplética $\omega$ qualquer, fixe o difeomorfismo local $\phi_{i}$ que leva $\theta^{i}$ a $\omega$, $i=0,1, \ldots, k$. Seja

$$
A_{k}^{i}=\phi_{i}^{-1}\left(A_{k}\right) .
$$

Suponha que $C$ é uma curva singular no espaço simplético $\left(\mathbb{R}^{2 n}, \omega\right)$ que é difeomorfo a $A_{k}$, então existe $f:\left(\mathbb{R}^{2 n}, 0\right) \longrightarrow\left(\mathbb{R}^{2 n}, 0\right)$ germe de difeomorfismo tal que $f\left(A_{k}\right)=C$ logo temos que $f^{*}[\omega]_{C}=\left[f^{*} \omega\right]_{A_{k}}$, isto diz que $[\omega]_{C}$ e $\left[f^{*} \omega\right]_{A_{k}}$ são difeomorfos, agora pelo anterior existe $i \in\{0,1, \ldots, k\}$ tal que $\left[f^{*} \omega\right]_{A_{k}}$ é difeomorfa $\left[A_{k}\right]^{i}$. Como $\left[A_{k}\right]^{i}$ é difeomorfo a $[\omega]_{A_{k}^{i}}$ segue que $[\omega]_{C}$ é difeomorfo a $[\omega]_{A_{k}^{i}}$, portanto o Teorema A (2) implica que $C$ é simplectomorfa a $A_{k}^{i}$.

Assim qualquer curva no espaço simplético $\left(\mathbb{R}^{2 n}, \omega\right)$ é simplectomorfa a uma e apenas uma das curvas $A_{k}^{0}, \ldots, A_{k}^{k}$. Isso dá a classificação obtida em [A].

Vejamos agora a demonstração do Teorema A. Para isso começamos com alguns lemas que são usados também depois.

Lema 2.19: $\quad$ Seja $(M, 0) \subset\left(\mathbb{R}^{m}, 0\right)$ uma subvariedade, $(N, 0) \subset(M, 0)$. Então existe um germe de submersão $\pi:\left(\mathbb{R}^{m}, 0\right) \rightarrow(M, 0)$ tal que $\pi(x)=x, \forall x \in M$. 
Demonstração: Como $M$ é subvariedade, existe uma carta $(\Psi, U)$ de $\mathbb{R}^{m}$ em $x=0$ tal que $\Psi: U \rightarrow \mathbb{R}^{m}, 0 \in U, \Psi(0)=0 \mathrm{e}$

$$
\Psi(U \cap M)=\left\{\left(x_{1}, x_{2}, \ldots, x_{s}, 0, \ldots, 0\right),-\epsilon_{i} \leqslant x_{i} \leqslant \epsilon, \epsilon_{i}>0,1 \leqslant i \leqslant s\right\} \text { onde } s=\operatorname{dim} M
$$

Posso considerar $U$ tal que $\Psi(U)$ contém um retângulo aberto

$$
V=\left(-\gamma_{1}, \gamma_{1}\right) \times\left(-\gamma_{2}, \gamma_{2}\right) \times \cdots \times\left(-\gamma_{m}, \gamma_{m}\right)
$$

Definimos $\tilde{\pi}: V \rightarrow \mathbb{R}^{s} \times\{0\}^{m-s}$, por $\tilde{\pi}\left(x_{1}, \ldots, x_{s}, x_{s+1}, \ldots, x_{m}\right)=\left(x_{1}, \ldots, x_{s}, 0, \ldots, 0\right)$.

Definimos $\pi=\Psi^{-1} \circ \tilde{\pi} \circ \Psi: \Psi^{-1}(V) \rightarrow M$, então $\pi$ satisfaz as condições requeridas.

Lema 2.20: Seja $N \subset \mathbb{R}^{l}$ um subconjunto, $W \subset T_{0} \mathbb{R}^{l}$ o espaço tangente de alguma subvariedade contendo $N$ de dimensão mínima entre tais subvariedades. Se w é uma p-forma com $[\omega]_{N}=0$ então $\left.\omega\right|_{W}=0$.

Demonstração: Fixe uma subvariedade $M$ contendo $N$ de dimensão minima entre tais subvariedades, tome $W=T_{0} M$. Pela Proposição 2.11 como $[\omega]_{N}=0$, temos que $\left[\left.\omega\right|_{T M}\right]_{N}=0$, isto é, existem $\alpha \in \Lambda_{N}^{p}(M), \beta \in \Lambda_{N}^{p-1}(M)$ e

$$
\omega=\alpha+d \beta
$$

Agora pela minimalidade da dimensão de $M, N$ não está contido em qualquer hipersuperfície de $M$, portanto qualquer função que se anula em $N$ tem 1-jato zero em 0 . De fato, se $f$ é uma função que se anula em $N$ com 1-jato não nulo, então $d_{0} f$ é submersão, logo $0=f(0)$ é um valor regular e portanto $f^{-1}(0)$ é uma hipersuperfície de $M$ que contém $N$.

Segue que $d \beta(0)=0$ e então $\left.\omega\right|_{T M}(0)=0$, isto é $\left.\omega\right|_{W}=\left.\omega\right|_{T_{0} M}=0$.

Lema 2.21: Seja $N \subset \mathbb{R}^{l}$ um subconjunto, $\omega$ uma 2-forma fechada em $\mathbb{R}^{l}$ com restrição algébrica zero a $N$. Seja $M \subset \mathbb{R}^{l}$ uma subvariedade contendo $N$ de dimensão mínima entre tais subvariedades. Então existe uma 2-forma fechada $\theta$ em $\mathbb{R}^{l}$ tal que $\left.\theta\right|_{T M}=\left.\omega\right|_{T M}$, $[\theta]_{N}=0$ e $\theta(0)=0$.

Demonstração: Seja $\mu=\left.\omega\right|_{T M}$, pela demonstração do Lema 2.20 temos $\mu(0)=0$. Seja $\pi$ como no Lema 2.19 e $\theta=\pi^{*} \mu$. Então $\theta$ é uma 2-forma fechada (pois $\omega$ é uma 2-forma fechada) que se anula em 0 (pois $\pi(0)=0$ ) e cuja restrição a $T M$ coincide com a restrição 
de $\omega$ a $T M\left(\left.\theta\right|_{T M}=\left.\omega\right|_{T M}\right)$, de fato:

$$
\left.\theta\right|_{T M}=\left.\left(\pi^{*} \mu\right)\right|_{T M}=\left.\mu\right|_{T M}=\left.\omega\right|_{T M},
$$

onde a penúltima igualdade segue de $\pi(x)=x, \forall x \in M$.

Desde que $[\omega]_{N}$ e $\left.\omega\right|_{T M}=\left.\theta\right|_{T M}$, pela Proposição 2.11 obtemos $[\theta]_{N}=[\omega]_{N}=0$.

A seguir provaremos o Teorema A, primeiro reduzimos o Teorema A (1) ao caso em que as formas simpléticas $\omega_{0}$ e $\omega_{1}$ satisfazem a condição $\left(\omega_{0}-\omega_{1}\right)(0)=0$ e depois provaremos o Teorema A neste caso particular.

Redução do Teorema A (1) ao caso $\left(\omega_{0}-\omega_{1}\right)(0)=0$

Tome $M \subset \mathbb{R}^{2 n}$ uma subvariedade contendo $N$ de dimensão mínima entre tais subvariedades (esta variedade existe pois o conjunto de todas as variedades que contém $N$ é não vazio). Pelo Lema 2.21 existe $\theta$, uma 2-forma fechada em $\mathbb{R}^{2 n}$ tal que $\left.\theta\right|_{T M}=\left.\omega_{0}\right|_{T M}-\left.\omega_{1}\right|_{T M}$, $[\theta]_{N}=0$ e $\theta(0)=0$. Seja $\tilde{\omega}=\omega_{1}+\theta$, então $\omega_{0}, \omega_{1}, \tilde{\omega}$ satisfazem as seguintes propriedades:

- $\left.\tilde{\omega}\right|_{T M}=\left.\omega_{0}\right|_{T M}$.

- $[\tilde{\omega}]_{N}=\left[\omega_{1}\right]_{N},\left(\tilde{\omega}-\omega_{1}\right)(0)=0$.

- $\tilde{\omega}$ é simplética.

A última propriedade segue dos seguintes fatos: i) $\theta(0)=0$ e $\tilde{\omega}$ é fechado. ii) Toda 2 -forma em $\mathbb{R}^{2 n}$ induz uma matriz quadrada de ordem $2 n$ que varia continuamente. iii) Uma 2-forma em $\mathbb{R}^{2 n}$ é simplética se e somente se a matriz induzida é invertível. iv) $G L(2 n)$ é um aberto do espaço de matrizes reais $2 n \times 2 n$.

Pelo Teorema 1.67 existe $\Phi:\left(\mathbb{R}^{2 n}, 0\right) \rightarrow\left(\mathbb{R}^{2 n}, 0\right)$ germe de difeomorfismo tal que $\Phi(x)=x, \forall x \in M$ e $\Phi^{*} \tilde{\omega}=\omega_{0}$.

Note que $\tilde{\omega}$ e $\omega_{1}$ satisfazem as condições do Teorema A (1) com a condição adicional $\left(\tilde{\omega}-\omega_{1}\right)(0)=0$, portanto o Teorema A (1) para as formas $\omega_{0}$ e $\omega_{1}$ é provado se provarmos este Teorema para as formas $\omega_{1}$ e $\tilde{\omega}$, com a condição $\left(\tilde{\omega}-\omega_{1}\right)(0)=0$.

Prova do Teorema A (1) no caso $\left(\omega_{0}-\omega_{1}\right)(0)=0$

Demonstração: Provaremos a existência de uma família de difeomorfismos $\Phi_{t}$ : $\left(\mathbb{R}^{2 n}, 0\right) \longrightarrow\left(\mathbb{R}^{2 n}, 0\right)$ que preservam pontualmente $N$ e $\Phi_{t}^{*}\left(\omega_{t}\right)=\omega_{0}$ onde $\omega_{t}=\omega_{0}+$ $t\left(\omega_{1}-\omega_{0}\right)$, para qualquer $t \in[0,1]$. Esta família será encontrada dentro das famílias que satisfazem a EDO

$$
\frac{d \Phi_{t}}{d t}=V_{t}\left(\Phi_{t}\right), \Phi_{0}=\mathrm{id}_{\mathbb{R}^{2 n}}
$$


onde $V_{t}$ é uma família de campos de vetores em $\mathbb{R}^{2 n}$ que se anulam em qualquer ponto de $N$ (o último implica que $\Phi_{t}$ preserva $N$ pontualmente).

Então é suficiente provar a existência destes campos de vetores. Lembramos que $\mathscr{L}_{V}$ é a derivada de Lie ao longo de um campo vetorial $V$.

A equação $\Phi_{t}^{*} \omega_{t}=\omega_{0}$ é equivalente à equação $\mathscr{L}_{V_{t}}\left(\omega_{t}\right)+\frac{d \omega_{t}}{d t}=0$ (de fato, é suficiente aplicar a equação (1.3) ). Da Fórmula de Cartan e do fato $\omega_{t}$ ser fechada obtemos

$$
d\left(i_{V_{t}}\left(\omega_{0}+t\left(\omega_{1}-\omega_{0}\right)\right)\right)=\omega_{0}-\omega_{1}
$$

Desde que $N$ é quase-homogêneo, pelo Teorema $2.15 H^{p}\left(N, \mathbb{R}^{2 n}\right)=0, p \geqslant 1$, assim para $p=2$ temos $\omega_{0}-\omega_{1}=d \beta$, onde $[\beta]_{N}=0$.

Portanto para resolver (2.7) é suficiente resolver a equação

$$
i_{V_{t}}\left(\omega_{0}+t\left(\omega_{1}-\omega_{0}\right)\right)=\beta
$$

Esta equação pode ser tratada como um sistema linear de equações nas variáveis $x \in \mathbb{R}^{2 n}$ próximo de 0 e $t \in[0,1]$.

A suposição $\left(\omega_{0}-\omega_{1}\right)(0)=0$ implica que $\left(\omega_{0}+t\left(\omega_{1}-\omega_{0}\right)\right)(0)=\omega_{0}(0)$ e como a forma $\omega_{0}$ é simplética temos que as 2 -formas $\left(\omega_{0}+t\left(\omega_{1}-\omega_{0}\right)\right)$ têm posto máximo $2 n$ para qualquer $t \in[0,1]$ e para qualquer $x$ próximo de 0 .

Portanto para tais $t$ e $x$, a matriz do sistema linear (2.8) é não-degenerada e consequentemente (2.8) tem solução única $V_{t}$, esta solução se anula em qualquer ponto de $N$ pois de $i_{V_{t}}\left(\omega_{t}\right)=\beta$ temos $\omega_{t}(x)\left(V_{t}(x), v\right)=\beta(x)(v) \quad \forall x \in \mathbb{R}^{2 n}, \forall v \in \mathbb{R}^{2 n}$, logo $\forall x \in N$

$$
\omega_{t}(x)\left(V_{t}(x), v\right)=\beta(x)(v)=0, \quad \forall v \in \mathbb{R}^{2 n} .
$$

Como a matriz do sistema linear (2.8) é não-degenerada obtemos $V_{t}(x)=0 \quad \forall x \in N$.

\section{Prova do Teorema A (2)}

Demonstração: Suponha primeiro $N_{1}$ e $N_{2}$ simplectomorfos, então existe $\Phi:\left(\mathbb{R}^{2 n}, 0\right) \rightarrow$ $\left(\mathbb{R}^{2 n}, 0\right)$ germe de difeomorfismo tal que $\Phi^{*} \omega=\omega$ e $\Phi\left(N_{1}\right)=N_{2}$, logo temos $\Phi^{*}\left([\omega]_{N_{2}}\right)=$ $\left[\Phi^{*} \omega\right]_{N_{1}}=[\omega]_{N_{1}}$ e portanto $[\omega]_{N_{1}}$ e $[\omega]_{N_{2}}$ são difeomorfos.

Agora suponha $[\omega]_{N_{1}}$ e $[\omega]_{N_{2}}$ são difeomorfos então existe $\Phi:\left(\mathbb{R}^{2 n}, 0\right) \rightarrow\left(\mathbb{R}^{2 n}, 0\right)$ germe de difeomorfismo tal que $\Phi^{*}\left([\omega]_{N_{2}}\right)=[\omega]_{N_{1}}$ e $\Phi\left(N_{1}\right) \subset N_{2}$ isto é $\left[\Phi^{*} \omega\right]_{N_{1}}=[\omega]_{N_{1}}$. Como $\Phi^{*} \omega$ é uma forma simplética, pelo Teorema A 1$)$, temos que existe $\Psi:\left(\mathbb{R}^{2 n}, 0\right) \rightarrow\left(\mathbb{R}^{2 n}, 0\right)$ germe de difeomorfismo tal que $\Psi(x)=x, \forall x \in N_{1}$ e $\Psi^{*}\left(\Phi^{*} \omega\right)=\omega$.

Assim $\Phi \circ \Psi$ é um germe de difeomorfismo que satisfaz $(\Phi \circ \Psi)^{*} \omega=\omega$ e $\Phi \circ \Psi\left(N_{1}\right) \subset N_{2}$. Portanto $N_{1}$ e $N_{2}$ são simplectomorfos. 
O significado geométrico dessa classificação é também uma aplicação do método das restrições algébricas. Como será mostrado abaixo.

Proposição 2.22: $\quad$ Se $N_{1}$ e $N_{2}$ são subconjuntos difeomorfos das variedades Lagrangianas $M_{1}$ e $M_{2}$ respectivamente num espaço simplético fixado $\left(\mathbb{R}^{2 n}, \omega\right)$, então $N_{1}$ e $N_{2}$ são simplectomorfos.

Mas como verificamos se um subconjunto de uma variedade simplética está contido numa subvariedade Lagrangiana?. Para responder essa pergunta temos o seguinte teorema.

Teorema 2.23 (Teorema B): Um subconjunto quase-homogêneo $N$ de um espaço simplético $\left(\mathbb{R}^{2 n}, \omega\right)$ está contido numa subvariedade Lagrangiana se e somente se $[\omega]_{N}=0$.

Antes de demonstrar o Teorema B vejamos uma aplicação.

Exemplo 2.24: Seja $C$ uma curva no espaço simplético $\left(\mathbb{R}^{2 n}, \omega\right)$, com $n \geqslant 2$ difeomorfa a $A_{k}$. Seja $A_{k}^{k}$ definida na equação (2.6). Pelo teorema $\mathrm{B}$, a curva $C$ está contida numa subvariedade Lagrangiana se e somente se $C$ é simplectomorfo a $A_{k}^{k}$.

De fato, primeiro suponha que $C$ está contida numa subvariedade Lagrangiana $M$, dos resultados da subseção anterior, temos que $C$ é simplectomorfo a $A_{k}^{i}$ para algum $i$. Note que pelo Teorema $\mathrm{B}, A_{k}^{k}$ está contida numa subvariedade Lagrangiana $M_{1}$ pois da equação (2.6) temos

$$
[\omega]_{A_{k}^{k}}=\phi_{k}^{*}\left(\left[\theta^{k}\right]_{A_{k}}\right)=\phi_{k}^{*}(0)=0 .
$$

Assim, $C$ é simplectomorfo a $A_{k}^{k}$ pois $[\omega]_{C}=[\omega]_{A_{k}^{k}}=0$.

Reciprocamente, suponha que $C$ é simplectomorfo a $A_{k}^{k}$, então existe $\phi:\left(\mathbb{R}^{2 n}, 0\right) \rightarrow$ $\left(\mathbb{R}^{2 n}, 0\right)$ germe de difeomorfismo tal que $\phi^{*} \omega=\omega$ e $\phi(C) \subset A_{k}^{k} \subset M_{1}$, $\log 0 \subset \subset$ $\phi^{-1}\left(M_{1}\right)=M_{2}$. Portanto será suficiente mostrar que $M_{2}$ é subvariedade Lagrangiana.

De $\operatorname{dim} M_{2}=\operatorname{dim} M_{1}=n$ (pois $\phi$ é difeomorfismo) e $i_{M_{2}}=\phi^{-1} \circ i_{M_{1}} \circ \phi$ onde $i_{M_{i}}:\left(M_{i}, 0\right) \longrightarrow\left(\mathbb{R}^{2 n}, 0\right), i=1,2$ são as inclusões segue

$$
i_{M_{2}}{ }^{*} \omega=\left(\phi^{-1} \circ i_{M_{1}} \circ \phi\right)^{*} \omega=\phi^{*}\left(i_{M_{1}}{ }^{*}\left(\phi^{-1 *}(\omega)\right)\right)=\phi^{*} i_{M_{1}}{ }^{*}(\omega)=\phi^{*}(0)=0 .
$$

Portanto $M_{2}$ é uma subvariedade Lagrangiana.

Agora vejamos a demonstração do Teorema B. 
Demonstração: Suponha que $M$ é uma subvariedade Lagrangiana de $\left(\mathbb{R}^{2 n}, \omega\right)$ tal que $N \subset M$. Considere a inclusão $i: M \rightarrow \mathbb{R}^{2 n}$ então $\left.\omega\right|_{T M}=\left.\left(i^{*} \omega\right)\right|_{T M}=0$ logo pela Proposição 2.11 temos $[\omega]_{N}=0$.

Reciprocamente, suponha $[\omega]_{N}=0$ e fixe uma subvariedade $M$ e uma 2-forma fechada $\theta$ como no Lema 2.21:

$$
\left.\theta\right|_{T M}=\left.\omega\right|_{T M}, \quad[\theta]_{N}=0, \quad \theta(0)=0,
$$

desde que $\theta(0)=0$ a forma $\omega-\theta$ é simplética (como na demonstração do Teorema A). Note que a variedade $M$ é isotrópica com respeito a $\omega-\theta$ (pois $\left.\theta\right|_{T M}=\left.\omega\right|_{T M}$ ).

Como $[\omega-\theta]_{N}=[\omega]_{N}$, pelo Teorema A 1) existe $\Phi:\left(\mathbb{R}^{2 n}, 0\right) \rightarrow\left(\mathbb{R}^{2 n}, 0\right)$ germe de difeomorfismo tal que

$$
\Phi(x)=x, \forall x \in N \text { e } \Phi^{*}(\omega)=\omega-\theta
$$

este difeomorfismo local leva $M$ numa subvariedade $\tilde{M}=\Phi(M)$ que contém $N$ e que é isotrópica com respeito a $\omega$, pois como $\left.\Phi^{*}(\omega)\right|_{T M}=\left.(\omega-\theta)\right|_{T M}=0$ então $\left.\omega\right|_{T \tilde{M}}=0$.

Agora do fato que quaisquer subvariendades isotrópica esta contida numa subvariedade Lagrangiana existe $P$ subvariedade Lagrangiana que contém $\tilde{M}$ o que implica $N \subset \Phi^{-1}(P)$, onde $\Phi^{-1}(P)$ é uma variedade Lagrangiana. 


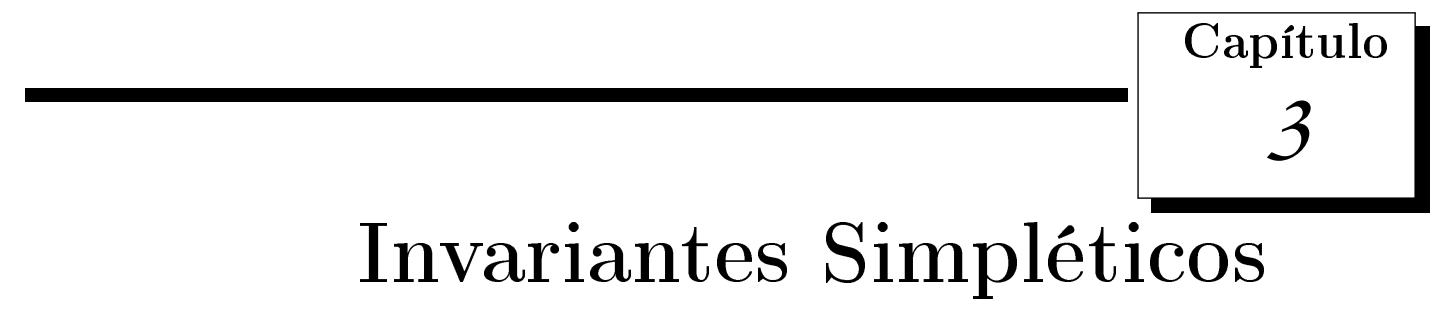

Lembremos que um invariante é aquelo que não muda em função de algumas operações, por exemplo o Número de Milnor é um invariante com respeito à $\mathcal{R}$-equivalência.

Nesta seção estudaremos os invariantes simpléticos, isto é, aqueles invariantes com respeito à ação do grupo de simetrias locais. O índice de isotropia foi definido por Arnol'd em [A], generalizaremos esta definição e expressaremos este invariante em termos da restrição algébrica. A multiplicidade simplética é definida como una generalização do caso de uma curva parametrizada [I-J] e também será descrito en função da restrição algébrica. Estas descripções são justamente os Teoremas C e D.

\subsection{Teorema $\mathrm{C}$ e $\mathrm{D}$}

Começemos com o índice de isotropia, mas antes de definir-lo precisamos saber o que é a ordem de anulamento.

Definição 3.1: Seja $\omega$ um germe de uma forma diferenciável em $\mathbb{R}^{2 n}$.

- Se $\omega$ tem $k$-1-jato zero e $k$-jato não zero, dizemos que $k$ é a ordem de anulamento de $\omega$.

- Se $\omega(0) \neq 0$ então dizemos que a ordem de anulamento de $\omega$ é zero.

- Se $\omega=0$ ou na $C^{\infty}$-categoria, $\omega$ tem expansão de Taylor zero, dizemos que a ordem de anulamento de $\omega$ é $\infty$.

Definição 3.2: Seja $N$ um subconjunto do espaço simplético $\left(\mathbb{R}^{2 n}, \omega\right)$. O indice de isotropia de $N$ é a ordem máxima de anulamento das 2-formas $\left.\omega\right|_{T M}$ sobre todas as subvariedades $M$ que contém $N$. 
Observação 3.3: A definição de índice de isotropia é equivalente a: a ordem máxima de tangência entre subvariedades que contém $N$ e subvariedades isotrópicas de mesma dimensão.

O índice de isotropia é zero se $N$ não está contida em qualquer subvariedade que é tangente a alguma subvariedade isotrópica de mesma dimensão. Se $N$ está contida numa subvariedade Lagrangiana, então o índice de isotropia é $\infty$.

Teorema 3.4 (Teorema C): $O$ indice de isotropia de uma variedade algébrica quasehomogênea $N$ num espaço simplético $\left(\mathbb{R}^{2 n}, \omega\right)$ é igual à ordem máxima de anulamento das 2-formas fechadas que representam a restrição algébrica $[\omega]_{N}$.

Como uma aplicação do Teorema C temos:

Exemplo 3.5: Sejam $A_{k}^{i}$ as curvas definidas em (2.6) no espaço simplético $\left(\mathbb{R}^{2 n}, \omega\right)$. Pelo Teorema C, o índice de isotropia de $A_{k}^{i}$ é $i$ se $i \leqslant k-1$, e o índice de isotropia de $A_{k}^{k}$ é $\infty$, pois está contida numa subvariedade Lagrangiana.

Demonstração: Para provar o Teorema C será suficiente provar as afirmações seguintes:

1. Se $M \subset\left(\mathbb{R}^{2 n}, \omega\right)$ é uma subvariedade contendo $N$ e tal que a restrição $\left.\omega\right|_{T M}$ tem $k$-jato zero, $k \geqslant 0$, então existe uma 2 -forma fechada $\tilde{\omega}$ em $\mathbb{R}^{2 n}$ com $k$-jato zero e $[\omega]_{N}=[\tilde{\omega}]_{N}$.

2. Se $\tilde{\omega}$ é uma 2 -forma fechada em $\mathbb{R}^{2 n}$ com $k$-jato zero, $k \geqslant 0$, tal que $[\omega]_{N}=[\tilde{\omega}]_{N}$, então existe uma subvariedade $M \subset \mathbb{R}^{2 n}$ contendo $N$ tal que a restrição $\left.\omega\right|_{T M}$ tem $k$-jato zero.

\section{Demonstração de 1)}

Seja $\pi:\left(\mathbb{R}^{2 n}, 0\right) \rightarrow(M, 0)$ um germe de submersão tal que $\pi(x)=x, \forall x \in M$, como no Lema 2.19 e $\tilde{\omega}=\pi^{*}\left(\left.\omega\right|_{T M}\right)$. Então temos $d \tilde{\omega}=d \pi^{*}\left(\left.\omega\right|_{T M}\right)=\pi^{*}\left(d\left(\left.\omega\right|_{T M}\right)\right)=\pi^{*}(0)=0$, pois $\omega$ é fechada, assim $\tilde{\omega}$ é uma 2 -forma fechada em $\mathbb{R}^{2 n}$ com $k$-jato zero. Por outro lado, note que $\left.\tilde{\omega}\right|_{T M}=\left.\left(\pi^{*}\left(\left.\omega\right|_{T M}\right)\right)\right|_{T M}=\left.\omega\right|_{T M}$ e portanto a Proposição 2.11 implica $[\tilde{\omega}]_{N}=[\omega]_{N}$.

\section{Demonstração de 2)}

Considere a forma $(\omega-\tilde{\omega})$. Esta é uma forma simplética (pois $\tilde{\omega}(0)=0)$ e $[\omega-\tilde{\omega}]_{N}=0$ então pelo Teorema B, $N$ está contida numa subvariedade Lagrangiana $M$ com respeito à forma simplética $(\omega-\tilde{\omega})$, isto significa que $\left.(\omega-\tilde{\omega})\right|_{T M}=0$. 
Portanto, desde que $\tilde{\omega}$ tem $k$-jato zero, sua restrição a $T M$ e, consequentemente, $\left.\omega\right|_{T M}$ tem $k$-jato zero.

Continuemos com a multiplicidade simplética de uma variedade algébrica num espaço simplético, para isso primeiro vejamos algumas definicições.

Definição 3.6: Seja $I$ um ideal no anel $\Lambda^{0}\left(\mathbb{R}^{m}\right)$.

- O conjunto de zeros de $I$ é o subconjunto de $\mathbb{R}^{m}$ consistindo dos pontos que anulam qualquer função de $I$.

- Dizemos que $I$ tem a propriedade de zeros se $I$ contém qualquer função que se anula em seu conjunto de zeros.

Observação 3.7: - No caso em que $I$ é um ideal com a propriedade dos zeros e $I=\langle H\rangle$ dizemos que $H$ tem a propriedade dos zeros.

- No que segue uma variedade algébrica em $\mathbb{R}^{m}$, significará o conjunto de zeros de algum ideal $k$-gerado tendo a propriedade de zeros $(k \geqslant 1)$.

Definição 3.8: Denote por $\operatorname{Var}(k, m)$ o espaço vetorial das variedades algébricas descritas por ideais $k$-gerados. Dado $N \in \operatorname{Var}(k, m)$ denote por $(N)$ a órbita de $N$ com respeito ao grupo dos difeomorfismos locais. A multiplicidade (ou número de Tjurina) de $N$ é a codimensão de $(N)$ em $\operatorname{Var}(k, m)$.

Observação 3.9: Para fazer precisa esta definição, deve se associar a $N$ um germe de função $H:\left(\mathbb{R}^{m}, 0\right) \rightarrow\left(\mathbb{R}^{k}, 0\right)$, cujas $k$ componentes são geradores do ideal de funções que se anulam em $N$. A órbita $(N)$ pode ser identificada com a órbita de $H$ com respeito à $\mathcal{K}$-equivalência (ou $V$-equivalência $[\mathrm{A}-\mathrm{V}-\mathrm{G}]$ ).

Em vista da definição anterior, definimos a multiplicidade simplética de uma variedade algébrica num espaço simplético como segue:

Definição 3.10: Seja $N$ uma variedade algébrica num espaço simplético $\left(\mathbb{R}^{2 n}, \omega\right)$. Seja $(N)$ a órbita de $N$ com respeito ao grupo dos difeomorfismos locais e seja $(N)^{\text {Symp }}$ a órbita de $N$ com respeito ao grupo dos simplectomorfismos locais. A multiplicidade simplética de $N$ é a codimensão de $(N)^{\text {Symp }}$ em $(N)$.

Observação 3.11: Seja $H:\left(\mathbb{R}^{2 n}, 0\right) \rightarrow\left(\mathbb{R}^{k}, 0\right)$ um germe como acima, ou seja, suas $k$ componentes geram o ideal de funções que se anulam em $N$. Seja $(H)_{\mathcal{K}}$ a órbita de $H$ com 


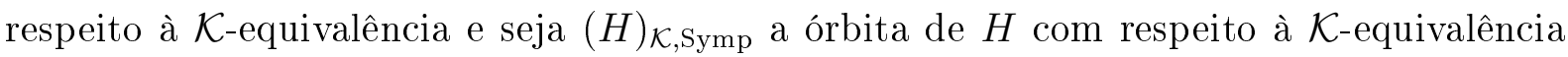
simplética. A $\mathcal{K}$-equivalência simplética é definida analogamente à $\mathcal{K}$-equivalência, a diferença é que a mudança de coordenadas na fonte é um simplectomorfismo local. A codimensão de $(N)^{\text {Symp }}$ em $(N)$ é a codimensão de $(H)_{\mathcal{K} \text {,Symp }}$ em $(H)_{\mathcal{K}}$.

Observação 3.12: Decorre do Teorema Clássico de Darboux outra definição equivalente de multiplicidade simplética de um subconjunto $N$ do espaço simplético $\left(\mathbb{R}^{2 n}, \omega\right)$ : é a codimensão da órbita de $\omega$ com respeito ao grupo de simetrias locais de $N$ no espaço de todas as 2-formas fechadas.

Portanto o Teorema A implica a seguinte teorema.

Teorema 3.13 (Teorema D): A multiplicidade simplética de uma variedade algébrica quase-homogênea num espaço simplético $\left(\mathbb{R}^{2 n}, \omega\right)$ é igual à codimensão da órbita da restrição algébrica $[\omega]_{N}$ com respeito ao grupo de simetrias locais de $N$ no espaço $\left[\Lambda^{2, \text { closed }}\left(\mathbb{R}^{2 n}\right)\right]_{N}$.

Vejamos uma aplicação deste teorema.

Exemplo 3.14: Sejam $A_{k}^{i}$ as curvas no espaço simplético $\left(\mathbb{R}^{2 n}, \omega_{0}\right)$ definidas em (2.6). No exemplo 2.13 provamos que a restrição algébrica $c_{0}\left[A_{k}\right]^{0}+\ldots+c_{k-1}\left[A_{k}\right]^{k-1}$ é difeomorfa a $\left[A_{k}\right]^{p}$ se e somente se $c_{0}=c_{1}=\ldots=c_{p-1}=0$ e $c_{p} \neq 0$. Portanto pelo Teorema $\mathrm{D}$ a multiplicidade simplética da curva $A_{k}^{i}$ é igual a $i$. Isto é satisfeito para todo $i \leqslant k$ (a curva $A_{k}^{k}$ corresponde à restrição algébrica zero que é no caso $c_{0}=c_{1}=\ldots=c_{k-1}=0$ ).

\section{$3.2 \mathrm{O}$ espaço $\left[\Lambda^{2, \operatorname{closed}}\left(\mathbb{R}^{2 n}\right)\right]_{N}$ e o conjunto $\left[\operatorname{Symp}\left(\mathbb{R}^{2 n}\right)\right]_{N}$}

Nesta seção estamos interessados em obter resultados envolvendo os conjuntos $\left[\Lambda^{2}\left(\mathbb{R}^{2 n}\right)\right]_{N}$, $\left[\Lambda^{2, \text { closed }}\left(\mathbb{R}^{2 n}\right)\right]_{N},\left[\operatorname{Symp}\left(\mathbb{R}^{2 n}\right)\right]_{N}$. Este estudo será feito brevemente e continuado no Capítulo 5 .

Em vista dos resultados da subseção prévia e com o objetivo de calcular uma base de $\left[\Lambda^{2, \text { closed }}\left(\mathbb{R}^{2 n}\right)\right]_{N}$ definimos

$$
s(N)=\operatorname{dim}_{\mathbb{R}}\left[\Lambda^{2, \operatorname{closed}}\left(\mathbb{R}^{2 n}\right)\right]_{N},
$$

vamos apresentar um resultado sobre $s(N)$, tendo como hipótese $s(N)<\infty$. Primeiro vejamos o seguinte lema.

Lema 3.15: Se $a \in\left[\Lambda^{2, \text { closed }}\left(\mathbb{R}^{2 n}\right)\right]_{N}$ e a $\neq 0$, então a órbita de a com respeito ao grupo de simetrias de $N$ tem dimensão maior ou igual que 1. 
Teorema 3.16: Seja $N$ uma variedade algébrica quase-homogênea num espaço simplético $\left(\mathbb{R}^{2 n}, \omega\right)$ tal que $s(N)<\infty$. Então a multiplicidade simplética de $N$ não excede $s(N)$, e é igual a $s(N)$ se e somente se $N$ está contida numa variedade Lagrangiana.

Demonstração: A primeira afirmação segue do Teorema D. Mostremos a segunda afirmação.

Pelo Teorema D a multiplicidade simplética é a codimensão da órbita da restrição algébrica $[\omega]_{N}$ em $\left[\Lambda^{2 \text {,closed }}\left(\mathbb{R}^{2 n}\right)\right]_{N}$. Segue do Lema 3.15 que a dimensão dessa órbita é maior o igual que 1 se $[\omega]_{N} \neq 0$, portanto a multiplicidade simplética é $s(N)$ se e somente se $[\omega]_{N}=0$. Pelo Teorema B, $[\omega]_{N}=0$ se e somente se $N$ está contido numa subvariedade Lagrangiana, portanto o resultado segue.

Agora queremos descrever o conjunto $\left[\operatorname{Symp}\left(\mathbb{R}^{2 n}\right)\right]_{N}$.

Teorema 3.17: Seja $N \subset \mathbb{R}^{2 n}$ um subconjunto, $r=\min \{\operatorname{dim} M / N \subset M$, M subvariedade\}. Seja $M_{0}$ uma subvariedade tal que $\operatorname{dim} M_{0}=r$ e $N \subset M_{0}$. A restrição algébrica $[\theta]_{N}$ de uma 2-forma fechada $\theta$ pode ser representada por uma forma simplética em $\mathbb{R}^{2 n}$ se e somente se posto $\left(\left.\theta\right|_{T_{0} M_{0}}\right) \geqslant 2 r-2 n$.

Demonstração: Segue do Teorema 1.70 que $\left.\operatorname{Symp}\left(\mathbb{R}^{2 n}\right)\right|_{\mathbb{R}^{r}}$ consiste das 2-formas fechadas em $\mathbb{R}^{r}$ de posto $\geqslant 2(r-n)$.

Suponhamos $[\theta]_{N} \in\left[\operatorname{Symp}\left(\mathbb{R}^{2 n}\right)\right]_{N}$, então existe $\alpha$, uma 2-forma simplética, tal que $[\theta]_{N}=[\alpha]_{N}$. Logo $[\theta-\alpha]_{N}=0$, então pelo Lema 2.20 temos $\left.(\theta-\alpha)\right|_{T_{0} M_{0}}=0$, consequentemente, $\left.\theta\right|_{T_{0} M_{0}}=\left.\alpha\right|_{T_{0} M_{0}}$ e portanto $\operatorname{posto}\left(\left.\theta\right|_{T_{0} M_{0}}\right)=\operatorname{posto}\left(\left.\alpha\right|_{T_{0} M_{0}}\right) \geqslant 2(r-n)$.

Agora suponha que posto $\left(\left.\theta\right|_{T_{0} M_{0}}\right) \geqslant 2 r-2 n$ então $\left.\left.\theta\right|_{T_{0} M_{0}} \in \operatorname{Symp}\left(\mathbb{R}^{2 n}\right)\right|_{M_{0}}$, portanto existe uma forma simplética $\alpha$ tal que $\left.\theta\right|_{T_{0} M_{0}}=\left.\alpha\right|_{T_{0} M_{0}}$, e portanto, pela Proposição 2.11 temos $[\theta]_{N}=[\alpha]_{N}$.

Proposição 3.18: Seja $N \subset \mathbb{R}^{2 n}$ um subconjunto.

1. Se $N$ está contido numa subvariedade 2-dimensional, então

$$
\left[\Lambda^{2, \text { closed }}\left(\mathbb{R}^{2 n}\right)\right]_{N}=\left[\Lambda^{2}\left(\mathbb{R}^{2 n}\right)\right]_{N}
$$

2. Se $N$ está contido numa subvariedade $n$-dimensional, então

$$
\left[\operatorname{Symp}\left(\mathbb{R}^{2 n}\right)\right]_{N}=\left[\Lambda^{2, \text { closed }}\left(\mathbb{R}^{2 n}\right)\right]_{N}
$$




\section{Demonstração:}

1. Por definição $\left[\Lambda^{2, \operatorname{closed}}\left(\mathbb{R}^{2 n}\right)\right]_{N} \subset\left[\Lambda^{2}\left(\mathbb{R}^{2 n}\right)\right]_{N}$.

Seja $[\omega]_{N} \in\left[\Lambda^{2}\left(\mathbb{R}^{2 n}\right)\right]_{N}$. Temos que existe $U \subset \mathbb{R}^{2 n}$ aberto que contém a origem e um sistema de coordenadas $x_{1}, x_{2}, \ldots, x_{2 n}$ tais que $U \cap N=\left\{\left(x_{1}, x_{2}, 0, \ldots, 0\right)\right\}$.

Do Teorema de Darboux $\left.\omega\right|_{T U}=\sum f_{i j} d x_{i} \wedge d x_{j}$, assim $\left[\left.\omega\right|_{T U}\right]_{N}=\left[\left(f_{12}-f_{21}\right) d x_{1} \wedge\right.$ $\left.d x_{2}\right]_{N}$, logo pela Proposição $2.11[\omega]_{N}=\left[\left(f_{12}-f_{21}\right) d x_{1} \wedge d x_{2}\right]_{N}$. Isto significa $[\omega]_{N} \in$ $\left[\Lambda^{2, \text { closed }}\left(\mathbb{R}^{2 n}\right)\right]_{N}$. Portanto $\left[\Lambda^{2, \text { closed }}\left(\mathbb{R}^{2 n}\right)\right]_{N}=\left[\Lambda^{2}\left(\mathbb{R}^{2 n}\right)\right]_{N}$.

2. É suficiente provar $\left[\Lambda^{2, \operatorname{closed}}\left(\mathbb{R}^{2 n}\right)\right]_{N} \subset\left[\operatorname{Symp}\left(\mathbb{R}^{2 n}\right)\right]_{N}$.

Sejam $[\omega]_{N} \in\left[\Lambda^{2, \operatorname{closed}}\left(\mathbb{R}^{2 n}\right)\right]_{N}, r=\min \{\operatorname{dim} M / N \subset M, M$ subvariedade $\}$ e $M_{0}$ uma subvariedade que contém $N$ de dimensão $r$. Por hipótese $r \leqslant n$ então posto $\left(\left.\omega\right|_{T_{0} M_{0}}\right) \geqslant$ $0=2 n-2 n \geqslant 2 r-2 n$, logo pelo Teorema 3.17, $[\omega]_{N} \in\left[\operatorname{Symp}\left(\mathbb{R}^{2 n}\right)\right]_{N}$.

Portanto $\left[\operatorname{Symp}\left(\mathbb{R}^{2 n}\right)\right]_{N}=\left[\Lambda^{2, \operatorname{closed}}\left(\mathbb{R}^{2 n}\right)\right]_{N}$.

Agora encontraremos uma base para $\left[\Lambda^{2, \operatorname{closed}}\left(\mathbb{R}^{2 n}\right)\right]_{N}$, quando $N$ é quase-homogêneo e o espaço $\left[\Lambda^{2}\left(\mathbb{R}^{2 n}\right)\right]_{N}$ tem dimensão finita.

Seja $\left\{a_{1}, \ldots, a_{k}\right\}$ uma base de $\left[\Lambda^{2}\left(\mathbb{R}^{2 n}\right)\right]_{N}$ e considere as restrições algébricas $\left\{d a_{1}, \ldots d a_{k}\right\}$ $\subset\left[\Lambda^{3}\left(\mathbb{R}^{2 n}\right)\right]_{N}$. Seja $p=\operatorname{dim}_{\mathbb{R}}\left(\mathbb{R}\left\{d a_{1}, \ldots d a_{k}\right\}\right), p \geqslant 0$. Reordenando $a_{1}, \ldots, a_{k}$ se necessário temos que:

1. As restrições algébricas $d a_{1}, \ldots d a_{p}$ são linearmente independentes.

Substituindo as restrições algébricas $a_{i}(p+1 \leqslant i \leqslant k)$, por $a_{i}+\sum_{j=1}^{p} k_{i j} a_{j}$ para convenientes $k_{i j} \in \mathbb{R}$, podemos supor que:

2. $d a_{p+1}=\ldots=d a_{k}=0$.

Teorema 3.19: Seja $N$ um subconjunto quase-homogêneo de $\mathbb{R}^{2 n}$ e $\left\{a_{1}, \ldots, a_{k}\right\}$ uma base de $\left[\Lambda^{2}\left(\mathbb{R}^{2 n}\right)\right]_{N}$ satisfazendo 1) e 2) acima. Então $\left\{a_{p+1}, \ldots, a_{k}\right\}$ é uma base do espaço $\left[\Lambda^{2, \text { closed }}\left(\mathbb{R}^{2 n}\right)\right]_{N}$.

Demonstração: Primeiro provemos que $\left\{a_{p+1}, \ldots, a_{k}\right\} \subset\left[\Lambda^{2, \text { closed }}\left(\mathbb{R}^{2 n}\right)\right]_{N}$.

De fato, fixe $i$ tal que $(p+1 \leqslant i \leqslant k)$. Seja $\omega_{i}$ um representante de $a_{i}$ então $a_{i}=\left[\omega_{i}\right]_{N}$. Desde que $N$ é quase-homogêneo, pelo Teorema $2.15 H^{3}\left(N, \mathbb{R}^{2 n}\right)=0$, isto quer dizer que para qualquer 3-forma $\omega$ que satisfaz $[\omega]_{N}=0$ e $d \omega=0$ existe uma 2-forma $\alpha$ tal que $[\alpha]_{N}=0$ e $d \alpha=\omega$. Assim em particular para a 3-forma $d \omega_{i}$ existe uma 2-forma $\tilde{\omega}_{i}$ tal que $d \omega_{i}=d \tilde{\omega}_{i}$ e $\left[\tilde{\omega}_{i}\right]_{N}=0$. 
Logo a 2-forma $\omega_{i}-\tilde{\omega}_{i}$ é fechada e $a_{i}=\left[\omega_{i}-\tilde{\omega}_{i}\right]$, portanto $a_{i} \in\left[\Lambda^{2, \operatorname{closed}}\left(\mathbb{R}^{2 n}\right)\right]_{N}$, para $p+1 \leqslant i \leqslant k$.

Agora provemos que $\left\{a_{p+1}, \ldots, a_{k}\right\}$ é base de $\left[\Lambda^{2, \operatorname{closed}}\left(\mathbb{R}^{2 n}\right)\right]_{N}$.

Seja $[\omega]_{N} \in\left[\Lambda^{2, \operatorname{closed}}\left(\mathbb{R}^{2 n}\right)\right]_{N} \subset\left[\Lambda^{2}\left(\mathbb{R}^{2 n}\right)\right]_{N}$, então $[\omega]_{N}=c_{1} a_{1}+\ldots+c_{k} a_{k}$, logo

$$
0=[d \omega]_{N}=c_{1} d a_{1}+\ldots+c_{p} d a_{p}+c_{p+1} d a_{p+1}+\ldots+c_{k} d a_{k}
$$

Segue de 1) e 2) que $c_{1}=c_{2}=\ldots=c_{p}=0$, portanto $[\omega]_{N} \in \mathbb{R}\left\{a_{p+1}, \ldots, a_{k}\right\}$.

Portanto $\mathbb{R}\left\{a_{p+1}, \ldots, a_{k}\right\}=\left[\Lambda^{2, \text { closed }}\left(\mathbb{R}^{2 n}\right)\right]_{N}$. 


\section{Capítulo} 4

\section{O método das restrições algébricas para curvas}

Resolver o Problema A, implica ter que classificar $\left[\Lambda^{2}\left(\mathbb{R}^{2}\right)\right]_{\{H=0\}}$ com respeito ao grupo de simetrias locais é por isso que neste capítulo restringimos nossos resultados a $\left[\Lambda^{2}\left(\mathbb{R}^{2}\right)\right]_{\{H=0\}}$, onde $H \in \Lambda^{0}\left(\mathbb{R}^{2}\right)$ é quase-homogêneo.

As duas primeiras seções é dedicado para que dado $a \in\left[\Lambda^{0}\left(\mathbb{R}^{2}\right)\right]_{\{H=0\}}$ obter uma maneira simples de calcular o espaço tangente a $a$. Na última seção provaremos dois teoremas fundamentais para fazer a classificação de $\left[\Lambda^{2}\left(\mathbb{R}^{2}\right)\right]_{\{H=0\}}$ com respeito ao grupo de simetrias locais. Esta classificação porém será feita no capítulo seguinte.

Definição 4.1: Um polinômio $H\left(x_{1}, x_{2}\right) \in \Lambda^{0}\left(\mathbb{R}^{2}\right)$ é quase-homogêneo se existem números positivos $\lambda_{1}, \lambda_{2}$ (pesos de quase-homogeneidade) e um número positivo $d$ (grau de quase-homogeneidade) tais que

$$
H\left(t^{\lambda_{1}} x_{1}, t^{\lambda_{2}} x_{2}\right)=t^{d} H\left(x_{1}, x_{2}\right), \forall t \in \mathbb{R}
$$

ou equivalentemente, se $H\left(x_{1}, x_{2}\right)$ é uma combinação linear de monômios $x_{1}^{\alpha_{1}} x_{2}^{\alpha_{2}}$ que satisfazem $\alpha_{1} \lambda_{1}+\alpha_{2} \lambda_{2}=d$.

Definição 4.2: Seja $H \in \Lambda^{0}\left(\mathbb{R}^{2}\right)$. O quociente $\frac{\Lambda^{0}\left(\mathbb{R}^{2}\right)}{\langle\nabla H\rangle}$ é chamado de álgebra local de $H$ e a dimensão deste espaço é chamada de multiplicidade de $H$ (note que na verdade é o número de Milnor de $H$ no capítulo 1).

Neste capítulo $H\left(x_{1}, x_{2}\right)$ é um polinômio quase-homogêneo com pesos $\lambda_{1}$ e $\lambda_{2}$ que tem a propriedade dos zeros e multiplicidade finita $\mu$. Também assumiremos que quaisquer germe em $\Lambda^{0}\left(\mathbb{R}^{2}\right)$ que seja quase-homogêneo, terá pesos $\lambda_{1}$ e $\lambda_{2}$. 
Lema 4.3: Seja $H=H\left(x_{1}, x_{2}\right)$ um polinômio quase-homogêneo com grau de quasehomogeneidade $\delta$. Então $H=\lambda_{1} \frac{x_{1}}{\delta} \frac{\partial H}{\partial x_{1}}+\lambda_{2} \frac{x_{2}}{\delta} \frac{\partial H}{\partial x_{2}}$.

Demonstração: De fato, derivando com respeito a $t$ na equação (4.1) e fazendo $t=1$ obtemos

$$
\lambda_{1} x_{1} \frac{\partial H}{\partial x_{1}}+\lambda_{2} x_{2} \frac{\partial H}{\partial x_{2}}=\delta H
$$

O seguinte lema caracteriza as 2-formas em $\mathbb{R}^{2}$ que têm restrição algébrica zero no caso em que $N$ é o conjunto de zeros de um polinômio quase-homogêneo.

Lema 4.4: Seja $H=H\left(x_{1}, x_{2}\right)$ um polinômio quase-homogêneo. Uma 2-forma $f d x_{1} \wedge$ $d x_{2}$ em $\mathbb{R}^{2}$ tem restrição algébrica zero à curva $\{H=0\}$ se e somente se $f \in\langle\nabla H\rangle$.

Demonstração: Primeiramente note que do Lema $4.3 H \in\langle\nabla H\rangle$ e por conseguinte $\langle H, \nabla H\rangle=\langle\nabla H\rangle$.

Suponha que $\left[f d x_{1} \wedge d x_{2}\right]_{\{H=0\}}=0$. Como $H$ tem a propriedade dos zeros, existem $A, B_{1}, B_{2} \in \Lambda^{0}\left(\mathbb{R}^{2}\right)$ tais que

$$
\begin{aligned}
f d x_{1} \wedge d x_{2} & =A H d x_{1} \wedge d x_{2}+d\left(H B_{1} d x_{1}+H B_{2} d x_{2}\right) \\
& =H\left(A-\frac{\partial B_{1}}{\partial x_{2}}+\frac{\partial B_{2}}{\partial x_{1}}\right) d x_{1} \wedge d x_{2}-B_{1} \frac{\partial H}{\partial x_{2}} d x_{1} \wedge d x_{2}+B_{2} \frac{\partial H}{\partial x_{1}} d x_{1} \wedge d x_{2}
\end{aligned}
$$

$\operatorname{logo} f \in\langle H, \nabla H\rangle=\langle\nabla H\rangle$.

Agora suponha que $f \in\langle\nabla H\rangle=\langle H, \nabla H\rangle$, ou seja existem $A, B, C \in \Lambda^{0}\left(\mathbb{R}^{2}\right)$ tais que $f=A H+\frac{\partial H}{\partial x_{1}} B+\frac{\partial H}{\partial x_{2}} C \log O$

$$
\begin{aligned}
f d x_{1} \wedge d x_{2} & =\left(A H+\frac{\partial H}{\partial x_{1}} B+\frac{\partial H}{\partial x_{2}} C\right) d x_{1} \wedge d x_{2} \\
& =H\left(A-\frac{\partial B}{\partial x_{1}}-\frac{\partial C}{\partial x_{2}}\right) d x_{1} \wedge d x_{2}+d\left(-H C d x_{1}+H B d x_{2}\right)
\end{aligned}
$$

em conclusão $\left[f d x_{1} \wedge d x_{2}\right]_{\{H=0\}}=0$.

\subsection{As simetrias infinitesimais da curva $\{H=0\}$}

Como já foi mencionado, queremos calcular o espaço tangente de $a \in\left[\Lambda^{2}\left(\mathbb{R}^{2}\right)\right]_{\{H=0\}}$, para isso nesta seção provamos que é possivel definir a derivada de Lie $\mathscr{L}_{V} a$, onde $V$ é um campo de vetores em $\Lambda^{0}\left(\mathbb{R}^{2}\right)$ que satisfaz algumas condições. Justamente esta derivada permite calcular o espaço tangente de $a$. 
Definição 4.5: Definimos o conjunto de simetrias infinitesimais da curva $\{H=0\}$, denotado por $\operatorname{Symm}^{\inf }(\{H=0\})$ ou $\operatorname{Derlog}\{H=0\}$ por

Derlog $\{H=0\}=\left\{V\right.$ é um campo de vetores em $\left.\mathbb{R}^{2} \mid V(H) \in\langle H\rangle\right\}$.

Observação 4.6: Note que $\operatorname{Derlog}\{H=0\}$ é um $\mathbb{R}$ espaço vetorial, além disso com respeito ao colchete de Lie este espaço é uma $\mathbb{R}$-álgebra.

Definição 4.7: Definimos os seguintes campos vetoriais em $\mathbb{R}^{2}$

O campo vetorial de Euler:

$$
E=\lambda_{1} x_{1} \frac{\partial}{\partial x_{1}}+\lambda_{2} x_{2} \frac{\partial}{\partial x_{2}}
$$

O campo vetorial Hamiltoniano:

$$
\mathscr{H}=\frac{\partial H}{\partial x_{2}} \frac{\partial}{\partial x_{1}}-\frac{\partial H}{\partial x_{1}} \frac{\partial}{\partial x_{2}} .
$$

Como consequência imediata do Lema 4.3 temos o seguinte lema

Lema 4.8: Seja $H=H\left(x_{1}, x_{2}\right)$ um polinômio quase-homogêneo com grau de quasehomogeneidade $\delta$. Então $E(H)=\delta H$.

Definição 4.9: Dizemos que uma 1-forma $\omega$ definida numa variedade $M^{n}$ possui a propriedade da divisão se para toda $p$-forma $\alpha$ em $M^{n}(1 \leqslant p \leqslant n-1)$ tal que $\omega \wedge \alpha=0$, existe uma $(p-1)$-forma $\beta$ em $M^{n}$ tal que $\alpha=\omega \wedge \beta$.

Teorema 4.10 (ver $[\mathrm{M}])$ : Seja $\omega=\sum_{i=1}^{n} f_{i} d x_{i}$ uma 1-forma em $\mathbb{R}^{n}$. Seja $I(\omega)=$ $\left\langle f_{1}, \ldots, f_{n}\right\rangle$. Se $\operatorname{dim} \frac{\Lambda^{0}\left(\mathbb{R}^{n}\right)}{I(\omega)}$ é finito, então $\omega$ tem a propriedade da divisão.

O seguinte Lema foi usado em muitos trabalhos como por exemplo em [A], [Ly].

Lema 4.11: Qualquer campo vetorial $V \in \operatorname{Derlog}\{H=0\}$ tem a forma $V=g_{1} E+g_{2} \mathscr{H}$ para alguns $g_{1}, g_{2} \in \Lambda^{0}\left(\mathbb{R}^{2}\right)$.

Demonstração: Seja $V \in \operatorname{Derlog}\{H=0\}$, então existe $R \in \Lambda^{0}\left(\mathbb{R}^{2}\right)$ tal que $V(H)=R H$. Pelo Lema $4.8 E(H)=\delta H$, onde $\delta$ é o grau de quase-homogeneidade de $H$.

Seja $V_{1}=V-\frac{R}{\delta} E$, então $V_{1}(H)=0$. Por outro lado existem $A, B \in \Lambda^{0}\left(\mathbb{R}^{2}\right)$ tais que $V_{1}=A \frac{\partial}{\partial x_{1}}+B \frac{\partial}{\partial x_{2}} \operatorname{logo}$ temos

$$
\begin{aligned}
\left(A d x_{2}-B d x_{1}\right) \wedge d H & =\left(A d x_{2}-B d x_{1}\right) \wedge\left(\frac{\partial H}{\partial x_{1}} d x_{1}+\frac{\partial H}{\partial x_{2}} d x_{2}\right) \\
& =\left(A \frac{\partial H}{\partial x_{1}}+B \frac{\partial H}{\partial x_{2}}\right) d x_{2} \wedge d x_{1}=V_{1}(H) d x_{2} \wedge d x_{1}=0 .
\end{aligned}
$$


Agora como $H$ tem multiplicidade finita, pelo Teorema $4.10 \mathrm{dH}$ tem a propriedade da divisão, isto implica que existe $R_{1} \in \Lambda^{0}\left(\mathbb{R}^{2}\right)$ tal que

$$
A d x_{2}-B d x_{1}=R_{1} d H=R_{1} \frac{\partial H}{\partial x_{1}} d x_{1}+R_{1} \frac{\partial H}{\partial x_{2}} d x_{2}
$$

Logo $R_{1} \mathscr{H}=R_{1} \frac{\partial H}{\partial x_{2}} \frac{\partial}{\partial x_{1}}-R_{1} \frac{\partial H}{\partial x_{1}} \frac{\partial}{\partial x_{2}}=A \frac{\partial}{\partial x_{1}}+B \frac{\partial}{\partial x_{2}}=V_{1}$ e por conseguinte $V=\frac{R}{\delta} E+R_{1} \mathscr{H}$.

No que segue $\mathscr{L}_{V}$ denota a derivada de Lie ao longo do campo vetorial $V$.

Lema 4.12: Sejam $V \in \operatorname{Derlog}\{H=0\}$ e $\theta \in \mathscr{A}_{0}^{2}\left(\{H=0\}, \mathbb{R}^{2}\right)$. Então $\mathscr{L}_{V}(\theta) \in$ $\mathscr{A}_{0}^{2}\left(\{H=0\}, \mathbb{R}^{2}\right)$.

Demonstração: Pela definição de $\mathscr{A}_{0}^{2}\left(\{H=0\}, \mathbb{R}^{2}\right)$, e o fato de $H$ ter a propriedade dos zeros temos $\theta=A H d x_{1} \wedge d x_{2}+d\left(B H d x_{1}+C H d x_{2}\right)$ onde $A, B, C \in \Lambda^{0}\left(\mathbb{R}^{2}\right)$, logo

$$
\begin{aligned}
\mathscr{L}_{V}(\theta)= & i_{V} d\left(A H d x_{1} \wedge d x_{2}+d\left(B H d x_{1}+C H d x_{2}\right)\right)+ \\
& +d\left(i_{V}\left(A H d x_{1} \wedge d x_{2}+d\left(B H d x_{1}+C H d x_{2}\right)\right)\right) \\
= & d\left(i_{V}\left(A H d x_{1} \wedge d x_{2}+d\left(B H d x_{1}+C H d x_{2}\right)\right)\right) \\
= & d\left(i_{V}\left(\left(A H+\frac{\partial(C H)}{\partial x_{1}}-\frac{\partial(B H)}{\partial x_{2}}\right) d x_{1} \wedge d x_{2}\right)\right)
\end{aligned}
$$

Primeiro provemos que o Lema é valido para $V=f E$ e $V=g \mathscr{H}$.

1. $V=g \mathscr{H}=g \frac{\partial H}{\partial x_{2}} \frac{\partial}{\partial x_{1}}-g \frac{\partial H}{\partial x_{1}} \frac{\partial}{\partial x_{2}}$. Temos

$$
\begin{aligned}
\mathscr{L}_{V}(\theta) & =d\left(i_{V}\left(\left(A H+\frac{\partial C H}{\partial x_{1}}-\frac{\partial B H}{\partial x_{2}}\right) d x_{1} \wedge d x_{2}\right)\right) \\
& =d\left(\left(g A H+g \frac{\partial C H}{\partial x_{1}}-g \frac{\partial B H}{\partial x_{2}}\right)\left(\frac{\partial H}{\partial x_{2}} d x_{2}-\left(-\frac{\partial H}{\partial x_{1}}\right) d x_{1}\right)\right) \\
& =d\left(\left(g A H+g \frac{\partial C H}{\partial x_{1}}-g \frac{\partial B H}{\partial x_{2}}\right) d H\right) \\
& =d\left(g A H+g \frac{\partial C H}{\partial x_{1}}-g \frac{\partial B H}{\partial x_{2}}\right) \wedge d H \\
& =-d\left(H d\left(g A H+g \frac{\partial C H}{\partial x_{1}}-g \frac{\partial B H}{\partial x_{2}}\right)\right) \in \mathscr{A}_{0}^{2}\left(\{H=0\}, \mathbb{R}^{2}\right)
\end{aligned}
$$

$\operatorname{logo} \mathscr{L}_{V}(\theta) \in \mathscr{A}_{0}^{2}\left(\{H=0\}, \mathbb{R}^{2}\right)$. 
2. $V=f E=\lambda_{1} x_{1} f \frac{\partial}{\partial x_{1}}+\lambda_{2} x_{2} f \frac{\partial}{\partial x_{2}}$

$$
\begin{aligned}
\mathscr{L}_{V}(\theta) & =d\left(i_{V}\left(\left(A H+\frac{\partial C H}{\partial x_{1}}-\frac{\partial B H}{\partial x_{2}}\right) d x_{1} \wedge d x_{2}\right)\right) \\
& =d\left(\left(f A H+f \frac{\partial C H}{\partial x_{1}}-f \frac{\partial B H}{\partial x_{2}}\right)\left(\lambda_{1} x_{1} d x_{2}-\lambda_{2} x_{2} d x_{1}\right)\right) \\
& =d\left(f A H\left(\lambda_{1} x_{1} d x_{2}-\lambda_{2} x_{2} d x_{1}\right)\right)+d(\tau)
\end{aligned}
$$

onde

$$
\begin{aligned}
\tau & =\left(f \frac{\partial C H}{\partial x_{1}}-f \frac{\partial B H}{\partial x_{2}}\right)\left(\lambda_{1} x_{1} d x_{2}-\lambda_{2} x_{2} d x_{1}\right) \\
& =\lambda_{1} x_{1} f \frac{\partial C H}{\partial x_{1}} d x_{2}-\lambda_{1} x_{1} f \frac{\partial B H}{\partial x_{2}} d x_{2}-\lambda_{2} x_{2} f \frac{\partial C H}{\partial x_{1}} d x_{1}+\lambda_{2} x_{2} f \frac{\partial B H}{\partial x_{2}} d x_{1}
\end{aligned}
$$

Agora das igualdades

$$
\begin{aligned}
& \lambda_{1} x_{1} f \frac{\partial B H}{\partial x_{2}} d x_{2}=\lambda_{1} d\left(x_{1} f B H\right)-\lambda_{1} \frac{\partial x_{1} f B H}{\partial x_{1}} d x_{1}-\lambda_{1} B H \frac{\partial x_{1} f}{\partial x_{2}} d x_{2} \\
& \lambda_{2} x_{2} f \frac{\partial C H}{\partial x_{1}} d x_{1}=\lambda_{2} d\left(x_{2} f C H\right)-\lambda_{2} \frac{\partial x_{2} f C H}{\partial x_{2}} d x_{2}-\lambda_{2} C H \frac{\partial x_{2} f}{\partial x_{1}} d x_{1}
\end{aligned}
$$

e fazendo

$$
\gamma=-\lambda_{1} d\left(x_{1} f B H\right)+\lambda_{1} B H \frac{\partial x_{1} f}{\partial x_{2}} d x_{2}-\lambda_{2} d\left(x_{2} f C H\right)+\lambda_{2} C H \frac{\partial x_{2} f}{\partial x_{1}} d x_{1},
$$

segue do Lema 4.8 que

$$
\begin{aligned}
\tau & =\lambda_{1} x_{1} f \frac{\partial C H}{\partial x_{1}} d x_{2}+\lambda_{1} \frac{\partial x_{1} f B H}{\partial x_{1}} d x_{1}+\lambda_{2} \frac{\partial x_{2} f C H}{\partial x_{2}} d x_{2}+\lambda_{2} x_{2} f \frac{\partial B H}{\partial x_{2}} d x_{1}+\gamma \\
& =\lambda_{1} x_{1} f \frac{\partial C H}{\partial x_{1}} d x_{2}+\lambda_{1} x_{1} f \frac{\partial B H}{\partial x_{1}} d x_{1}+\lambda_{2} x_{2} f \frac{\partial C H}{\partial x_{2}} d x_{2}+\lambda_{2} x_{2} f \frac{\partial B H}{\partial x_{2}} d x_{1}+\gamma^{\prime} \\
& =\lambda_{1} x_{1} f C \frac{\partial H}{\partial x_{1}} d x_{2}+\lambda_{1} x_{1} f B \frac{\partial H}{\partial x_{1}} d x_{1}+\lambda_{2} x_{2} f C \frac{\partial H}{\partial x_{2}} d x_{2}+\lambda_{2} x_{2} f B \frac{\partial H}{\partial x_{2}} d x_{1}+\tilde{\gamma} \\
& =f C\left(\lambda_{1} x_{1} \frac{\partial H}{\partial x_{1}}+\lambda_{2} x_{2} \frac{\partial H}{\partial x_{2}}\right) d x_{2}+f B\left(\lambda_{1} x_{1} \frac{\partial H}{\partial x_{1}}+\lambda_{2} x_{2} \frac{\partial H}{\partial x_{2}}\right) d x_{1}+\tilde{\gamma} \\
& =f C(\delta H) d x_{2}+f B(\delta H) d x_{1}+\tilde{\gamma}
\end{aligned}
$$

onde

$$
\begin{aligned}
& \gamma^{\prime}=\gamma+\lambda_{1} B H \frac{\partial x_{1} f}{\partial x_{1}} d x_{1}+\lambda_{2} C H \frac{\partial x_{2} f}{\partial x_{2}} d x_{2}, \\
& \tilde{\gamma}=\gamma^{\prime}+\lambda_{1} x_{1} f H \frac{\partial C}{\partial x_{1}} d x_{2}+\lambda_{1} x_{1} f H \frac{\partial B}{\partial x_{1}} d x_{1}+\lambda_{2} x_{2} f H \frac{\partial C}{\partial x_{2}} d x_{2}+\lambda_{2} x_{2} f H \frac{\partial B}{\partial x_{2}} d x_{1},
\end{aligned}
$$

e $\delta$ é o grau de quase-homogeneidade de $H$.

Portanto $\mathscr{L}_{V}(\theta) \in \mathscr{A}_{0}^{2}\left(\{H=0\}, \mathbb{R}^{2}\right)$. 
Logo a afirmação segue de 1), 2) e do Lema 4.11.

Do Lema 4.12 segue que podemos definir a derivada de Lie de uma restrição algébrica $a=[\omega]_{\{H=0\}} \in\left[\Lambda^{2}\left(\mathbb{R}^{2}\right)\right]_{\{H=0\}}$ ao longo de um campo vetorial $V \in \operatorname{Derlog}\{H=0\}$ por $\mathscr{L}_{V}(a)=\left[\mathscr{L}_{V}(\omega)\right]_{\{H=0\}}$.

Com esta definição temos o seguinte resultado.

Lema 4.13: $\mathscr{L}_{g \mathscr{H}}(a)=0$ para qualquer $g \in \Lambda^{0}\left(\mathbb{R}^{2}\right)$ e para qualquer $a \in\left[\Lambda^{2}\left(\mathbb{R}^{2}\right)\right]_{\{H=0\}}$.

Demonstração: Seja $a=\left[F\left(x_{1}, x_{2}\right) d x_{1} \wedge d x_{2}\right]$ e $\theta=\mathscr{L}_{g} \mathscr{H}\left(F d x_{1} \wedge d x_{2}\right)$. Temos que

$$
i_{\mathscr{H}}\left(d x_{1} \wedge d x_{2}\right)=i_{\frac{\partial H}{\partial x_{2}} \frac{\partial}{\partial x_{1}}-\frac{\partial H}{\partial x_{1}} \frac{\partial}{\partial x_{2}}}\left(d x_{1} \wedge d x_{2}\right)=\frac{\partial H}{\partial x_{2}} d x_{2}+\frac{\partial H}{\partial x_{1}} d x_{1}=d H .
$$

Isto implica

$$
\begin{aligned}
\theta & =\mathscr{L}_{g \mathscr{H}}\left(F d x_{1} \wedge d x_{2}\right)=d\left(i_{g \mathscr{H}} F d x_{1} \wedge d x_{2}\right)+i_{g \mathscr{H}}\left(d\left(F d x_{1} \wedge d x_{2}\right)\right) \\
& =d\left(i_{g \mathscr{H}} F d x_{1} \wedge d x_{2}\right)=d\left(g F i_{\mathscr{H}}\left(d x_{1} \wedge d x_{2}\right)\right) \\
& =d(g F d H)=d(d(g F H)-H d(g F)) \\
& =-d(H d(g F))
\end{aligned}
$$

onde $H d(g F) \in \Lambda_{\{H=0\}}^{1}\left(\mathbb{R}^{2}\right)$ portanto $[\theta]_{\{H=0\}}=0$

Notação: Denotamos por $T(a)$ o espaço tangente em $a$ à órbita da restrição algébrica $a$. A seguinte proposição é uma consequência dos Lemas 4.11 e 4.13.

Proposição 4.14: Seja $a \in\left[\Lambda^{2}\left(\mathbb{R}^{2}\right)\right]_{\{H=0\}}$. Então $T(a)=\left\{\mathscr{L}_{g E}(a), g \in \Lambda^{0}\left(\mathbb{R}^{2}\right)\right\}$.

Demonstração: Provaremos $T(a) \subset\left\{\mathscr{L}_{g E}(a), g \in \Lambda^{0}\left(\mathbb{R}^{2}\right)\right\}$. Seja $a=[\theta]_{\{H=0\}}$.

Seja $\varphi_{t}=\left(\varphi_{t}^{1}, \varphi_{t}^{2}\right)$ uma família de simetrias locais de $\{H=0\}, t \in(-1,1)$, tal que $\varphi_{0}=i d_{\mathbb{R}^{2}}$. Seja $V_{t}$ família de campo de vetores associada à família $\varphi_{t}\left(\left.\frac{\partial \varphi_{r}}{\partial r}\right|_{r=t}=V_{t}\left(\varphi_{t}\right)\right)$.

Segue de (1.3) que

$$
\left.\frac{d}{d t} \varphi_{t}^{*} \theta\right|_{t=0}=\left.\varphi_{t}^{*}\left(\mathscr{L}_{V_{t}} \theta\right)\right|_{t=0}+\left.\frac{d \theta}{d t}\right|_{t=0}=\varphi_{0}^{*}\left(\mathscr{L}_{V_{0}} \theta\right)=\mathscr{L}_{V_{0}} \theta
$$

e portanto $\left.\frac{d}{d t} \varphi_{t}^{*} a\right|_{t=0}=\mathscr{L}_{V_{0}} a$.

Suponha que $V_{0}=\left(f_{1}, f_{2}\right)$, onde $f_{1}, f_{2} \in \lambda^{0}\left(\mathbb{R}^{2}\right)$. Note que para todo $x \in\{H=0\}$ temos $H \circ \varphi(x)=0, \forall t \in(-1,1)$, então para cada $t \in(-1,1)$ existe $u_{t} \in \lambda^{0}\left(\mathbb{R}^{2}\right)$ tais que

$$
H \circ \varphi_{t}=H . u_{t},
$$




$$
\begin{aligned}
\left.\operatorname{logo} \frac{d}{d t}\left(H \circ \varphi_{t}\right)\right|_{t=0} & =\left.H \cdot \frac{d u_{t}}{d t}\right|_{t=0} \text { isto é, } \\
\left.H \cdot \frac{d u_{t}}{d t}\right|_{t=0} & =\frac{\partial H}{\partial x_{1}} \frac{d \varphi_{t}^{1}}{d t}+\left.\frac{\partial H}{\partial x_{2}} \frac{d \varphi_{t}^{2}}{d t}\right|_{t=0}=\frac{\partial H}{\partial x_{1}} f_{1} \circ \varphi_{0}+\frac{\partial H}{\partial x_{2}} f_{2} \circ \varphi_{0}=V_{0}(H),
\end{aligned}
$$

pois $\left.\frac{\partial \varphi_{t}}{\partial t}\right|_{t=0}=V_{0}\left(\varphi_{0}\right)=\left(f_{1} \circ \varphi_{0}, f_{2} \circ \varphi_{0}\right)$.

Portanto $V_{0} \in \operatorname{Derlog}\{H=0\}$ e assim pelos Lemas 4.11 e 4.13, $T(a) \subset\left\{\mathscr{L}_{g E}(a), g \in\right.$ $\left.\Lambda^{0}\left(\mathbb{R}^{2}\right)\right\}$.

\subsection{Restrições algébricas de curvas quase-homogêneas}

Agora calculamos o espaço tangente $T(a)$ de forma mais explícita. Isto requer trabalhar com restrições algébricas de curvas quase-homogêneas. A possibilidade de definir restrições algébricas de curvas quase-homogêneas segue do seguinte lema.

Notação: Dado $F \in \Lambda^{0}\left(\mathbb{R}^{2}\right)$ denote por $F^{(\delta)}$ a parte quase-homogênea de grau $\delta$ de sua série de Taylor com respeito aos pesos $\lambda_{1}, \lambda_{2}$.

Observação 4.15: Seja $F \in \Lambda^{0}\left(\mathbb{R}^{2}\right)$, note que se $\delta \notin\left\{\alpha_{1} \lambda_{1}+\alpha_{2} \lambda_{2} / \alpha_{1}, \alpha_{2} \in \mathbb{N}\right\}$ então $F^{(\delta)}=0$, pois o grau de quase-homogeneidade é sempre da forma $a_{1} \lambda_{1}+a_{2} \lambda_{2}$, para alguns $a_{1}, a_{2} \in \mathbb{N}$.

Lema 4.16: $S e\left[F d x_{1} \wedge d x_{2}\right]_{\{H=0\}}=0$ então $\left[F^{(d)} d x_{1} \wedge d x_{2}\right]_{\{H=0\}}=0$ para qualquer $d$.

Demonstração: Seja $\delta$ o grau de quase-homogeneidade de $H$. Note que derivando com respeito a $x_{1}$ e $x_{2}$ a equação (4.1) obtemos que $\frac{\partial H}{\partial x_{1}}$ e $\frac{\partial H}{\partial x_{2}}$ são polinômios quase-homogêneos de graus $\delta-\lambda_{1}$ e $\delta-\lambda_{2}$ respectivamente.

Agora pelo Lema $4.4 F \in(\nabla H)$, isto implica que existem $f_{1}, f_{2} \in \Lambda^{0}\left(\mathbb{R}^{2}\right)$ tais que

$$
F=f_{1} \frac{\partial H}{\partial x_{1}}+f_{2} \frac{\partial H}{\partial x_{2}}
$$

Note agora que

$$
F^{(d)}=\left(f_{1} \frac{\partial H}{\partial x_{1}}\right)^{(d)}+\left(f_{2} \frac{\partial H}{\partial x_{2}}\right)^{(d)}
$$

$\mathrm{e}$

$$
F\left(t^{\lambda_{1}} x_{1}, t^{\lambda_{2}} x_{2}\right)=t^{\delta-\lambda_{1}} f_{1}\left(t^{\lambda_{1}} x_{1}, t^{\lambda_{2}} x_{2}\right) \frac{\partial H}{\partial x_{1}}\left(x_{1}, x_{2}\right)+t^{\delta-\lambda_{2}} f_{2}\left(t^{\lambda_{1}} x_{1}, t^{\lambda_{2}} x_{2}\right) \frac{\partial H}{\partial x_{2}}\left(x_{1}, x_{2}\right)
$$

o que implica

$$
F^{(d)}=f_{1}^{\left(d+\lambda_{1}-\delta\right)} \frac{\partial H}{\partial x_{1}}+f_{2}^{\left(d+\lambda_{2}-\delta\right)} \frac{\partial H}{\partial x_{2}}
$$


logo pelo Lema $4.4 \quad\left[F^{(d)} d x_{1} \wedge d x_{2}\right]_{\{H=0\}}=0$.

Definição 4.17: Seja $F=F\left(x_{1}, x_{2}\right)$ e $a=\left[F d x_{1} \wedge d x_{2}\right]_{\{H=0\}}$. A restrição algébrica

$$
a^{(d)}=\left[F^{\left(d-\lambda_{1}-\lambda_{2}\right)} d x_{1} \wedge d x_{2}\right]_{\{H=0\}}
$$

é chamada de parte quase-homogênea de grau $d$ de $a$. Se $a=a^{(d)}$ então $a$ é quasehomogênea de grau $d$.

O porque de $F^{\left(d-\lambda_{1}-\lambda_{2}\right)}$ e não $F^{(d)}$ na definição acima é dada pelo seguinte resultado:

Lema 4.18: Se uma restrição algébrica $a \in\left[\Lambda^{2}\left(\mathbb{R}^{2}\right)\right]_{\{H=0\}}$ é quase-homogênea de grau $d_{0}$ ent $\tilde{a} o \mathscr{L}_{E}(a)=d_{0} a$.

Demonstração: Seja $a=\left[F d x_{1} \wedge d x_{2}\right]_{\{H=0\}}$. Note que

$$
\mathscr{L}_{E}\left(F d x_{1} \wedge d x_{2}\right)=d\left(i_{E} F d x_{1} \wedge d x_{2}\right)+i_{E}\left(d\left(F d x_{1} \wedge d x_{2}\right)\right)=d\left(i_{E} F d x_{1} \wedge d x_{2}\right),
$$

que implica

$$
\mathscr{L}_{E}\left(F d x_{1} \wedge d x_{2}\right)=\left(\lambda_{1} x_{1} \frac{\partial F}{\partial x_{1}}+\lambda_{2} x_{2} \frac{\partial F}{\partial x_{2}}\right) d x_{1} \wedge d x_{2}+\left(\lambda_{1}+\lambda_{2}\right) F d x_{1} \wedge d x_{2}
$$

Agora da hipótese $\left[F d x_{1} \wedge d x_{2}\right]_{\{H=0\}}=\left[F^{\left(d_{0}-\lambda_{1}-\lambda_{2}\right)} d x_{1} \wedge d x_{2}\right]_{\{H=0\}}$ e do Lema 4.4 existem $g_{1}, g_{2} \in \Lambda^{0}\left(\mathbb{R}^{2}\right)$ tais que

$$
F-F^{\left(d_{0}-\lambda_{1}-\lambda_{2}\right)}=g_{1} \frac{\partial H}{\partial x_{1}}+g_{2} \frac{\partial H}{\partial x_{2}} .
$$

Derivando com respeito a $x_{1}$ e $x_{2}$ a equação (4.3), depois multiplicando por $\lambda_{1} x_{1} \mathrm{e}$ $\lambda_{2} x_{2}$ respectivamente e somando estas últimas equações obtemos

$$
\lambda_{1} x_{1} \frac{\partial F}{\partial x_{1}}+\lambda_{2} x_{2} \frac{\partial F}{\partial x_{2}}-\left(d_{0}-\lambda_{1}-\lambda_{2}\right) F^{\left(d_{0}-\lambda_{1}-\lambda_{2}\right)} \in\langle\nabla H\rangle .
$$

Segue das equações (4.2), (4.4) e do Lema 4.4 que

$$
\begin{aligned}
\mathscr{L}_{E}(a) & =\left[\left(d_{0}-\lambda_{1}-\lambda_{2}\right) F^{\left(d_{0}-\lambda_{1}-\lambda_{2}\right)} d x_{1} \wedge d x_{2}\right]_{\{H=0\}}+\left(\lambda_{1}+\lambda_{2}\right) a \\
& =\left(d_{0}-\lambda_{1}-\lambda_{2}\right) a+\left(\lambda_{1}+\lambda_{2}\right) a \\
& =d_{0} a .
\end{aligned}
$$

Lema 4.19: Para qualquer $a \in\left[\Lambda^{2}\left(\mathbb{R}^{2}\right)\right]_{\{H=0\}}$, a soma $\sum_{d \in \mathbb{R}} a^{(d)}$ é finita. 
Demonstração: Da Observação $4.15 a^{(d)}=0$ se $d \notin\left\{\alpha_{1} \lambda_{1}+\alpha_{2} \lambda_{2} / \alpha_{1}, \alpha_{2} \in \mathbb{N}\right\}$, portanto é suficiente provar que $a^{(d)}=0$ para $d$ suficientemente grande.

Seja $d>\lambda_{1}+\lambda_{2}+\mu\left(\lambda_{1}+\lambda_{2}\right)$ onde $\mu$ é a multiplicidade de $H$ então $a^{(d)}=\left[F d x_{1} \wedge\right.$ $\left.d x_{2}\right]_{\{H=0\}}$ onde $F \in \Lambda^{0}\left(\mathbb{R}^{2}\right)$ tem $\mu$-jato zero, pois se $x_{1}^{\alpha} x_{2}^{\beta}$ é um monômio de $F$ temos $\alpha \lambda_{1}+\beta \lambda_{2}=d-\lambda_{1}-\lambda_{2}>\mu\left(\lambda_{1}+\lambda_{2}\right), \operatorname{logo} \alpha+\beta>\mu$. Consequentemente $F \in \mathscr{M}^{\mu} \subset(\nabla H)$, portanto pelo Lema $4.4 a^{(d)}=0$.

Em vista da Proposição 4.14, vamos calcular a derivada de Lie $\mathscr{L}_{g E}(a)$ para quasehomogêneos $g \in \Lambda^{0}\left(\mathbb{R}^{2}\right)$ e $a \in\left[\Lambda^{2}\left(\mathbb{R}^{2}\right)\right]_{\{H=0\}}$.

Lema 4.20: Se $a \in\left[\Lambda^{2}\left(\mathbb{R}^{2}\right)\right]_{\{H=0\}}$ e $g \in \Lambda^{0}\left(\mathbb{R}^{2}\right)$ são quase-homogêneos de graus $\delta_{1}, \delta_{2}$ respectivamente, então $\mathscr{L}_{g E}(a)=\left(\delta_{1}+\delta_{2}\right)$ ga.

Demonstração: Temos que para quaisquer $\omega \in \Lambda^{2}\left(\mathbb{R}^{2}\right), g \in \Lambda^{0}\left(\mathbb{R}^{2}\right)$ e qualquer campo vetorial $V$ em $\mathbb{R}^{2}$

$$
\mathscr{L}_{g V}(\omega)=g \mathscr{L}_{V}(\omega)+\left(\mathscr{L}_{V}(g)\right) \omega
$$

Então o resultado segue da Equação 4.5 e dos Lemas 4.8 e 4.18.

Os Lemas 4.16 e 4.19, garantem que a seguinte transformação linear está bem definida.

Definição 4.21: Seja $Q$ a transformação linear

$$
Q:\left[\Lambda^{2}\left(\mathbb{R}^{2}\right)\right]_{\{H=0\}} \longrightarrow\left[\Lambda^{2}\left(\mathbb{R}^{2}\right)\right]_{\{H=0\}} \text {, definida por } Q(a)=\sum_{\delta \in \mathbb{R}} \delta a^{(\delta)}
$$

Proposição 4.22: Seja $a \in\left[\Lambda^{2}\left(\mathbb{R}^{2}\right)\right]_{\{H=0\}}$, considerando $\langle a\rangle$ como um $\mathbb{R}$-espaço vetorial temos $T(a)=Q(\langle a\rangle)$.

Proposição 4.23: Seja $H\left(x_{1}, x_{2}\right)$ um polinômio quase-homogêneo de multiplicidade finita $\mu$ tendo a propriedade dos zeros. Seja $a \in\left[\Lambda^{2}\left(\mathbb{R}^{2}\right)\right]_{\{H=0\}}$, então considerando $\langle a\rangle$ como um $\mathbb{R}$-espaço vetorial temos

$$
\operatorname{dim}_{\mathbb{R}} T(a)=\operatorname{dim}_{\mathbb{R}}\langle a\rangle .
$$

Demonstração: Seja $a=\left[F d x_{1} \wedge d x_{2}\right]_{\{H=0\}}$. Pela Proposição 4.22 temos $T(a)=$ $Q(\langle a\rangle)$. Assim será suficiente provar que $Q$ é injetora.

Suponha que $\sum \delta a^{(\delta)}=0$ logo existe $n \in \mathbb{N}$ tal que $\sum_{i=1}^{n} \delta_{i} a^{\left(\delta_{i}\right)}=0$, aplicando $\mathscr{L}_{E}$ 
$(n-1)$-vezes a esta equação obtemos o sistema não singular

$$
\left\{\begin{array}{cccccc}
\delta_{1} a^{\left(\delta_{1}\right)} & + & \cdots & + & \delta_{n} a^{\left(\delta_{n}\right)} & =0 \\
\delta_{1}^{2} a^{\left(\delta_{1}\right)} & + & \cdots & + & \delta_{n}^{2} a^{\left(\delta_{n}\right)} & =0 \\
\vdots & & \vdots & & \vdots & \vdots \\
\delta_{1}^{n} a^{\left(\delta_{1}\right)} & + & \cdots & + & \delta_{n}^{n} a^{\left(\delta_{n}\right)} & =0
\end{array}\right.
$$

e portanto $a^{\left(\delta_{i}\right)}=0$ para todo $1 \leqslant i \leqslant n$, $\log 0 F^{\delta-\lambda_{1}-\lambda_{2}} \in\langle\nabla H\rangle$ para todo $\delta$. Agora pela Proposição $1.47 \mathscr{M}_{2}^{\mu} \subset\langle\nabla H\rangle$ e pela Observação $1.45 F-j^{\mu-1} F(0) \in \mathscr{M}_{2}^{\mu}$. Então

$$
F=j^{\mu-1} F(0)+g, \quad g \in\langle\nabla H\rangle
$$

Pela definição de $F^{\delta-\lambda_{1}-\lambda_{2}}$, temos que se for preciso, adicionamos monômios de grau maior que $\mu$ a $j^{\mu-1} F(0)$ tais que a equação (4.6) tem a forma

$$
F=\sum_{\text {finita }} F^{\delta-\lambda_{1}-\lambda_{2}}+\tilde{g}, \quad \tilde{g} \in\langle\nabla H\rangle
$$

portanto $F \in\langle\nabla H\rangle$ e $a=0$.

\subsection{Ferramentas para a Classificação}

Para finalizar o capítulo provaremos duas proposições que são as ferramentas que serão usadas na classificação de $\left[\lambda^{2}\left(\mathbb{R}^{2}\right)\right]_{\{H=0\}}$ com respeito ao grupo de simetrias locais de $\{H=0\}$. Usaremos a seguinte notação.

Notação: Denote por $\circ(\delta)$ o subespaço de $\left[\Lambda^{2}\left(\mathbb{R}^{2}\right)\right]_{\{H=0\}}$ que consiste das restrições algébricas sem termos quase-homogêneos de grau menor ou igual que $\delta$.

Proposição 4.24: Suponha que exista um base $a_{1}, \ldots, a_{\mu}$ de $\left[\Lambda^{2}\left(\mathbb{R}^{2}\right)\right]_{\{H=0\}}$ que consiste de restrições algébricas quase-homogêneas de graus $\delta_{1} \leqslant \delta_{2} \leqslant \ldots \leqslant \delta_{\mu}$. Seja a $=c_{1} a_{1}+$ $\cdots+c_{\mu} a_{\mu}$. Se $a_{s}$ pertence ao espaço afim $g \cdot\left(c_{1} a_{1}+\cdots+c_{s-1} a_{s-1}\right)+\circ\left(\delta_{s}\right)$ para algum $g \in \Lambda^{0}\left(\mathbb{R}^{2}\right)$ tal que $g(0)=0$, então a é difeomorfa a uma restrição algébrica no espaço afim $c_{1} a_{1}+\cdots+c_{s-1} a_{s-1}+\circ\left(\delta_{s}\right)$.

Demonstração: Provaremos que uma simetria $\Psi$ da curva $\{H=0\}$ que reduz $a$ à forma normal requerida está contida no fluxo $\Phi^{t}$ do campo vetorial $g E$.

Como $g(0)=0$ temos pelo Lema de Hadamard que existem $f_{1}, f_{2} \in \Lambda^{0}\left(\mathbb{R}^{2}\right)$ tais 
que $g(x)=x_{1} f_{1}(x)+x_{2} f_{2}(x)$, logo segue da Equação 4.5 que

$$
\begin{aligned}
\mathscr{L}_{g E}(a) & =\mathscr{L}_{g E}\left(c_{1} a_{1}+\cdots+c_{s} a_{s}+\cdots+c_{\mu} a_{\mu}\right) \\
& \in \mathscr{L}_{g E}\left(c_{1} a_{1}+\cdots+c_{s-1} a_{s-1}\right)+\mathscr{L}_{g E}\left(c_{s} a_{s}\right)+\circ\left(\delta_{s}\right) \\
& \in \mathscr{L}_{g E}\left(c_{1} a_{1}+\cdots+c_{s-1} a_{s-1}\right)+c_{s}\left(g \mathscr{L}_{E}\left(a_{s}\right)+\mathscr{L}_{E}(g) a_{s}\right)+\circ\left(\delta_{s}\right) \\
& \in \mathscr{L}_{g E}\left(c_{1} a_{1}+\cdots+c_{s-1} a_{s-1}\right)+c_{s} g \delta_{s} a_{s}+c_{s}\left(\lambda_{1} x_{1} \frac{\partial g}{\partial x_{1}}+\lambda_{2} x_{2} \frac{\partial g}{\partial x_{2}}\right) a_{s}+\circ\left(\delta_{s}\right) \\
& \in \mathscr{L}_{g E}\left(c_{1} a_{1}+\cdots+c_{s-1} a_{s-1}\right)+\circ\left(\delta_{s}\right)
\end{aligned}
$$

Agora da definição de $\mathscr{L}_{V}$ para restrições algébricas temos

$$
\begin{aligned}
\mathscr{L}_{g E}\left(c_{1} a_{1}+\cdots+c_{s-1} a_{s-1}\right) & =d i_{g E}\left(c_{1} a_{1}+\cdots+c_{s-1} a_{s-1}\right)+i_{g E} d\left(c_{1} a_{1}+\cdots+c_{s-1} a_{s-1}\right) \\
& =d i_{g E}\left(c_{1} a_{1}+\cdots+c_{s-1} a_{s-1}\right) \\
& =d i_{E}\left(g \cdot\left(c_{1} a_{1}+\cdots+c_{s-1} a_{s-1}\right)\right) \\
& =d i_{E}\left(g \cdot\left(c_{1} a_{1}+\cdots+c_{s-1} a_{s-1}\right)\right)+i_{E} d\left(g \cdot\left(c_{1} a_{1}+\cdots+c_{s-1} a_{s-1}\right)\right) \\
& =\mathscr{L}_{E}\left(g \cdot\left(c_{1} a_{1}+\cdots+c_{s-1} a_{s-1}\right)\right)
\end{aligned}
$$

portanto, da hipótese $a_{s} \in g \cdot\left(c_{1} a_{1}+\cdots+c_{s-1} a_{s-1}\right)+\circ\left(\delta_{s}\right)$ segue que

$$
\begin{aligned}
\delta_{s} a_{s} & \in \mathscr{L}_{E}\left(g \cdot\left(c_{1} a_{1}+\cdots+c_{s-1} a_{s-1}\right)\right)+\circ\left(\delta_{s}\right) \\
& \in \mathscr{L}_{g E}\left(c_{1} a_{1}+\cdots+c_{s-1} a_{s-1}\right)+\circ\left(\delta_{s}\right)
\end{aligned}
$$

por conseguinte $\mathscr{L}_{g E}(a) \in \delta_{s} a_{s}+\circ\left(\delta_{s}\right)$, e logo

$$
\frac{d\left(\Phi^{t}\right)^{*} a}{d t} \in\left(\Phi^{t}\right)^{*}\left(\delta_{s} a_{s}+\circ\left(\delta_{s}\right)\right) .
$$

Note agora que para qualquer $t$, o difeomorfismo $\Phi^{t}$ preserva os eixos $x_{1}$ e $x_{2}$ e desde que $g(0)=0$, então $\Phi^{t}$ tem 1-jato igual a identidade. Estas propriedades implicam que $\Phi^{t}$ preserva o espaço afim $\delta_{s} a_{s}+\circ\left(\delta_{s}\right)$ e consequentemente $\frac{d\left(\Phi^{t}\right)^{*} a}{d t} \in \delta_{s} a_{s}+\circ\left(\delta_{s}\right)$. Agora $\Phi_{0}=i d$, segue que $\left(\Phi^{t}\right)^{*} a=a+t \delta_{s} a_{s}+\circ\left(\delta_{s}\right)$. Seja $t_{0}=\frac{-c_{s}}{\delta_{s}}$. Então $\Phi^{t_{0}}$ é a simetria requerida.

Proposição 4.25: Suponha que exista um base $a_{1}, \ldots, a_{\mu}$ de $\left[\Lambda^{2}\left(\mathbb{R}^{2}\right)\right]_{\{H=0\}}$ que consiste de restrições algébricas quase-homogêneas de graus $\delta_{1}<\delta_{2}<\ldots<\delta_{\mu}$ respectivamente. Qualquer restrição algébrica da forma $a=c_{p} a_{p}+\cdots+c_{\mu} a_{\mu}$ com $c_{p} \neq 0$ é difeomorfa $a$ uma restrição algébrica da forma $\tilde{a}=c_{p} a_{p}+\tilde{c}_{p+1} a_{p+1} \cdots+\tilde{c}_{\mu} a_{\mu}$, onde $\tilde{c}_{i}=0$ para todo $i \geqslant p+1$ tal que $a_{i} \in\left\langle a_{p}\right\rangle$. 
Demonstração: Suponha que $a_{s}=g \cdot a_{p}$ para algum $g \in \Lambda^{0}\left(\mathbb{R}^{2}\right)$ e $s \geqslant p+1$. Então

$$
0=a_{s}^{(p)}=\left(g . a_{p}\right)^{(p)}=g(0) a_{p}
$$

que implica $g(0)=0$ pois $a_{p}$ é linearmente independente. Segue da Proposição 4.24 que $a$ é difeomorfa a uma restrição algébrica no espaço afim $c_{p} a_{p}+\ldots+c_{s-1} a_{s-1}+\circ\left(\delta_{s}\right)$.

Sejam $s_{1}, s_{2}, \ldots, s_{l} \in \mathbb{N}$ tais que $a_{s_{i}}=g_{i} . a_{p}, s_{i}<s_{j}$ se $i<j$ e $g_{i} \in \Lambda^{0}\left(\mathbb{R}^{2}\right), i=1, \ldots, l$. Então $a$ é difeomorfo a uma restrição algébrica do espaço afim

$$
c_{1} a_{1}+\ldots+c_{s_{1}-1} a_{s_{1}-1}+\circ\left(\delta_{s_{1}}\right)
$$

Repetindo este argumento para $s_{2}$ obtemos que $a$ é difeomorfo a uma restrição algébrica do espaço afim

$$
c_{1} a_{1}+\ldots+c_{s_{1}-1} a_{s_{1}-1}+c_{s_{1}+1} a_{s_{1}+1}+\ldots+c_{s_{2}-1} a_{s_{2}-1}+\circ\left(\delta_{s_{2}}\right)
$$

Continuando este procedimento obtemos o resultado. 
Capítulo

Classificação simplética de curvas planas quase-homogêneas singulares

Nesta seção consideremos o germe de uma curva plana quase-homogênea singular em $\mathbb{R}^{2 n}$

$$
N=\left\{H\left(x_{1}, x_{2}\right)=x_{\geqslant 3}=0\right\} \subset \mathbb{R}^{2 n}
$$

onde $H \in \Lambda^{0}\left(\mathbb{R}^{2}\right)$ satisfaz as seguintes condições:

1. $H(0)=0, d H(0)=0$.

2. A propriedade dos zeros.

3. O germe $H$ é um polinômio quase-homogêneo de pesos $\lambda_{1}, \lambda_{2}$ e grau $d$.

Exemplo 5.1: Os clássicos exemplos são os seguintes germes simples de funções

$$
\begin{aligned}
& A_{k}: H\left(x_{1}, x_{2}\right)=x_{1}^{k+1}-x_{2}^{2}, k \geqslant 1 ; \\
& D_{k}: H\left(x_{1}, x_{2}\right)=x_{1}^{2} x_{2}-x_{2}^{k-1}, k \geqslant 4 \\
& E_{6}: H\left(x_{1}, x_{2}\right)=x_{1}^{3}-x_{2}^{4} \\
& E_{7}: H\left(x_{1}, x_{2}\right)=x_{1}^{3}-x_{1} x_{2}^{3} \\
& E_{8}: H\left(x_{1}, x_{2}\right)=x_{1}^{3}-x_{2}^{5}
\end{aligned}
$$

Nesta seção mostramos que o espaço vetorial $\left[\Lambda^{2, \operatorname{closed}}\left(\mathbb{R}^{2 n}\right)\right]_{N}$ pode ser identificado com a álgebra local do germe $H\left(x_{1}, x_{2}\right)$. 


\subsection{O espaço das restrições algébricas}

Teorema 5.2: Seja $N=\left\{H\left(x_{1}, x_{2}\right)=x_{\geqslant 3}=0\right\}$ um germe de curva plana quasehomogênea, onde $H$ tem multiplicidade finita $\mu$, e seja $\left\{f_{1}, f_{2}, \ldots, f_{\mu}\right\}$ uma base de $\frac{\Lambda^{0}\left(\mathbb{R}^{2}\right)}{\langle\nabla H\rangle}$ onde $f_{1}(0) \neq 0, f_{\geqslant 2}(0)=0$. Então

1. $\left[\Lambda^{2, \text { closed }}\left(\mathbb{R}^{2 n}\right)\right]_{N}$ é um espaço vetorial $\mu$-dimensional gerado pelas restrições algébricas $a_{i}=\left[f_{i} d x_{1} \wedge d x_{2}\right]_{N}, i=1, \ldots, \mu$.

2. Se $n \geqslant 2$ então $\left[\operatorname{Symp}\left(\mathbb{R}^{2 n}\right)\right]_{N}=\left[\Lambda^{2, \operatorname{closed}}\left(\mathbb{R}^{2 n}\right)\right]_{N}$. A variedade $\left[\operatorname{Symp}\left(\mathbb{R}^{2}\right)\right]_{N}$ consiste das restrições algébricas da forma $c_{1} a_{1}+\ldots+c_{\mu} a_{\mu}, c_{1} \neq 0$.

\section{Demonstração:}

1. Primeiro vejamos que $\left[\Lambda^{2, \text { closed }}\left(\mathbb{R}^{2 n}\right)\right]_{N}=\mathbb{R}\left\{a_{1}, \ldots, a_{\mu}\right\}$. Seja $[\omega]_{N} \in\left[\Lambda^{2, \text { closed }}\left(\mathbb{R}^{2 n}\right)\right]_{N}$, como $N \subset \mathbb{R}^{2} \times\{0\}^{2 n-2}=M$ temos $\left[\left.\omega\right|_{T M}\right]_{N}=\left[f\left(x_{1}, x_{2}\right) d x_{1} \wedge d x_{2}\right]_{N}$ para algum $f \in$ $\Lambda^{0}\left(\mathbb{R}^{2}\right)$, assim pela Proposição $2.11[\omega]_{N}=\left[f\left(x_{1}, x_{2}\right) d x_{1} \wedge d x_{2}\right]_{N}$. Logo pelo fato de $\left\{f_{1}, f_{2}, \ldots, f_{\mu}\right\}$ ser base da álgebra local temos que existem $\alpha_{i}, i=1, \ldots, \mu$, e $g_{1}, g_{2} \in$ $\Lambda^{0}\left(\mathbb{R}^{2}\right)$ tais que

$$
f=\sum_{i=1}^{\mu} \alpha_{i} f_{i}+g_{1} \frac{\partial H}{\partial x_{1}}+g_{2} \frac{\partial H}{\partial x_{2}} .
$$

Segue então do Lema 4.4 que $[\omega]_{N}=\left[f\left(x_{1}, x_{2}\right) d x_{1} \wedge d x_{2}\right]_{N}=\sum_{i=1}^{\mu} \alpha_{i} a_{i}$. Portanto $\left[\Lambda^{2, \text { closed }}\left(\mathbb{R}^{2 n}\right)\right]_{N}=\mathbb{R}\left\{a_{1}, a_{2}, \ldots, a_{\mu}\right\}$.

Agora provemos que $\left\{a_{1}, a_{2}, \ldots, a_{\mu}\right\}$ é linearmente independente. Suponha $0=\sum_{i=1}^{\mu} \alpha_{i} a_{i}$ para $\alpha_{i} \in \mathbb{R}, i=0, \ldots, \mu$, então $\left[\sum_{i=1}^{\mu} \alpha_{i} f_{i} d x_{1} \wedge d x_{2}\right]_{N}=0$. Segue do Lema 4.4 que $\sum \alpha_{i} f_{i} \in\langle\nabla H\rangle$, que implica $\sum_{i=1}^{\mu} \alpha_{i} f_{i}=0 \in \frac{\Lambda^{0}\left(\mathbb{R}^{2}\right)}{\langle\nabla H\rangle}$, portanto $\alpha_{i}=0, i=0, \ldots, \mu$, ou seja $\left\{a_{1}, a_{2}, \ldots, a_{\mu}\right\}$ é linearmente independente.

2. Primeiro considere $n \geqslant 2$. Neste caso pela Proposição 3.18 temos que $\left[\operatorname{Symp}\left(\mathbb{R}^{2 n}\right)\right]_{N}=$ $\left[\Lambda^{2, \text { closed }}\left(\mathbb{R}^{2 n}\right)\right]_{N}$ pois $N \subset \mathbb{R}^{n} \times\{0\}$.

Agora considere $n=2$ e seja $\omega=f d x_{1} \wedge d x_{2}$. Se $[\omega]_{N} \in\left[\operatorname{Symp}\left(\mathbb{R}^{2}\right)\right]_{N} \subset\left[\Lambda^{2, \text { closed }}\left(\mathbb{R}^{2}\right)\right]_{N}$, então existem $c_{i} \in \mathbb{R}, i=1, \ldots, \mu$, tais que

$$
[\omega]_{N}=\left[\sum c_{i} f_{i} d x_{1} \wedge d x_{2}\right]_{N}
$$

logo pelo Lema 4.4 existem $g_{1}, g_{2} \in \Lambda^{0}\left(\mathbb{R}^{2}\right)$ tais que

$$
f-\sum c_{i} f_{i}=g_{1} \frac{\partial H}{\partial x_{1}}+g_{2} \frac{\partial H}{\partial x_{2}}
$$

isto implica $\omega(0)=c_{1} f_{1}(0) d x_{1} \wedge d x_{2}$ pois $d H(0)=0$. Logo como $\omega$ é simplética temos $c_{1} f_{1}(0) \neq 0$ que implica $c_{1} \neq 0$. 
Reciprocamente, seja $[\omega]_{N}=\left[\sum_{i=0}^{\mu} c_{i} f_{i} d x_{1} \wedge d x_{2}\right]_{N}$ tal que $c_{1} \neq 0$. Analogamente temos que $\omega(0)=c_{1} f_{1}(0) d x_{1} \wedge d x_{2}$ isto implica posto $\left(\left.\omega\right|_{T_{0} \mathbb{R}^{2}}\right)=2 \geqslant 2(2-1)$, além disso $\omega$ é fechada, então pelo Teorema 1.70 temos que $[\omega]_{N} \in\left[\operatorname{Symp}\left(\mathbb{R}^{2}\right)\right]_{N}$.

Observação 5.3: Se $H$ não é quase-homogêneo, então da prova do Lema 4.4 o espaço $\left[\Lambda^{2, \text { closed }}\left(\mathbb{R}^{2 n}\right)\right]_{N}$ pode ser idenficado com o espaço $\frac{\Lambda^{0}\left(\mathbb{R}^{2}\right)}{\langle\nabla H, H\rangle}$.

\section{$5.2 \mathrm{O}$ índice de isotropia e a multiplicidade simplética}

O índice de isotropia e a multiplicidade simplética foram definidos no capítulo 3, agora vejamos como calculá-los.

Notação: Seja $P=\left\{x \in \mathbb{R}^{2 n}: x_{\geqslant 3}=0\right\} \subset \mathbb{R}^{2 n}$. Dado uma 2-forma $\omega$ em $\mathbb{R}^{2 n}$ denote por $F_{\omega}=F_{\omega}\left(x_{1}, x_{2}\right)$ o germe tal que o pullback de $\omega$ ao plano $P$ tem a forma $F_{\omega} d x_{1} \wedge d x_{2}$ isto significa $i_{P}^{*} \omega=F_{\omega} d x_{1} \wedge d x_{2}$, onde $i_{P}$ é inclusão.

Teorema 5.4: Seja $N=\left\{H\left(x_{1}, x_{2}\right)=x_{\geqslant 3}=0\right\}$ um germe de curva plana quasehomogênea singular no espaço simplético $\left(\mathbb{R}^{2 n}, \omega\right)$. O índice de isotropia de $N$ é o máximo $p \in \mathbb{N}$ tal que $F_{\omega} \in\langle\nabla H\rangle+\mathscr{M}^{p}$, onde $\mathscr{M}=\mathscr{M}_{2}$ é o ideal máximal de $\Lambda^{0}\left(\mathbb{R}^{2}\right)$. Se $F_{\omega} \in\langle\nabla H\rangle$ então $p=\infty$, se $F_{\omega}(0) \neq 0$ então $p=0$.

\section{Demonstração:}

- $F_{\omega} \in\langle\nabla H\rangle$ implica $\left[\left.\omega\right|_{T P}\right]_{N}=\left[i_{P}{ }^{*} \omega\right]_{N}=\left[F_{\omega} d x_{1} \wedge d x_{2}\right]_{N}=0$ portanto $[\omega]_{N}=0 \mathrm{e}$ logo pelo Teorema B, $N$ está contida numa subvariedade Lagrangiana. Então pela Observação 3.3 o índice de isotropia é infinito.

- Se $F_{\omega}(0) \neq 0$, segue da Definição 3.1.

- Seja $p<\infty$ o índice de isotropia de $N$ e $p_{0}$ o máximo inteiro positivo tal que $F_{\omega} \in\langle\nabla H\rangle+\mathscr{M}^{p_{0}}$.

Seja $\theta$ uma 2-forma fechada tal que sua ordem de anulamento é $p$ e tal que $[\omega]_{N}=$ $[\theta]_{N}$. Então $\left[\left.\omega\right|_{T P}\right]_{N}=\left[\left.\theta\right|_{T P}\right]_{N}$ e logo $\left[\left(F_{\omega}-F_{\theta}\right) d x_{1} \wedge d x_{2}\right]_{N}=0$, assim pelo Lema $4.4 F_{\omega}-F_{\theta} \in\langle\nabla H\rangle$.

Agora segue do fato que $F_{\theta}$ tem $(p-1)$-jato zero que $F_{\theta} \in \mathscr{M}^{p}$. Portanto $F_{\omega} \in$ $\langle\nabla H\rangle+\mathscr{M}^{p}$ e $p \leqslant p_{0}$. 
Recíprocamente, se $F_{\omega} \in\langle\nabla H\rangle+\mathscr{M}^{p_{0}}$, então

$$
F_{\omega}=f_{1} \frac{\partial H}{\partial x_{1}}+f_{2} \frac{\partial H}{\partial x_{2}}+\sum g_{i_{1}, \ldots, i_{p_{0}}} x_{i_{1} \ldots x_{i_{0}}} .
$$

Logo pelo Lema 4.4

$$
\left[\left.\omega\right|_{T P}\right]_{N}=\left[\sum \tilde{g}_{i_{1}, \ldots, i_{p_{0}}} x_{i_{1} \ldots} x_{i_{p_{0}}} d x_{1} \wedge d x_{2}\right]_{N}
$$

onde $\tilde{g}=g\left(x_{1}, x_{2}, 0, \ldots, 0\right)$, consequentemente

$$
[\omega]_{N}=\left[\sum \tilde{g}_{i_{1}, \ldots, i_{p_{0}}} x_{i_{1} \ldots x_{i_{0}}} d x_{1} \wedge d x_{2}\right]_{N}
$$

Note que $\sum \tilde{g}_{\left(i_{1}, \ldots, i_{p_{0}}\right)} x_{i_{1} \ldots x_{i_{0}}} d x_{1} \wedge d x_{2}$, é uma 2-forma fechada, portanto pelo Teorema $\mathrm{C}$ obtemos $p_{0} \leqslant p$. Assim $p=p_{0}$

No que segue, consideremos o índice de isotropia finito.

Teorema 5.5: Seja $N=\left\{H\left(x_{1}, x_{2}\right)=x_{\geqslant 3}=0\right\}$ um germe de curva plana quasehomogênea singular no espaço simplético $\left(\mathbb{R}^{2 n}, \omega\right)$. Seja $\mu$ a multiplicidade de $H$.

1. O indice de isotropia de $N$ não excede $(\mu-1)$ a menos que $N$ esteja contida numa subvariedade Lagrangiana (no último caso, o indice é infinito).

2. A multiplicidade simplética de $N$ não excede $\mu$, e é igual a $\mu$ se e somente se $N$ está contida numa subvariedade Lagrangiana.

\section{Demonstração:}

1. Suponha que $N$ não está contida em uma subvariedade Lagrangiana, então pelo Teorema B, $[\omega]_{N} \neq 0$, logo pela Proposição $2.11\left[\left.\omega\right|_{T M}\right]_{N} \neq 0$, para $M=\mathbb{R}^{2} \times\{0\}$. O último implica que $\left[F_{\omega} d x_{1} \wedge d x_{2}\right]_{N}=\left[i_{M}{ }^{*} \omega\right]_{N}=\left[\left.\omega\right|_{T M}\right]_{N} \neq 0$, onde $i_{M}$ é a inclusão, logo pelo Lema $4.4 F_{\omega} \notin\langle\nabla H\rangle$.

Por outro lado desde que $\mathscr{M}^{\mu} \subset\langle\nabla H\rangle$, temos $F_{\omega} \notin\langle\nabla H\rangle+\mathscr{M}^{\mu}$ e pelo Teorema 5.5 , o índice não excede $\mu-1$.

2. Pelo Teorema 3.16, $s(N)=\operatorname{dim}\left[\Lambda^{2, \text { closed }}\left(\mathbb{R}^{2 n}\right)\right]_{N}=\mu<\infty$ e desde que $H$ é quasehomogêneo pelo Teorema 5.2 temos que a multiplicidade simplética não excede $\mu$. Além disso é igual a $\mu$ se e somente se $N$ está contido numa subvariedade Lagrangiana. 
Proposição 5.6: Seja $H\left(x_{1}, x_{2}\right)$ um polinômio quase-homogêneo de multiplicidade finita tendo a propriedade dos zeros. A codimensão no espaço $\left[\Lambda^{2}\left(\mathbb{R}^{2}\right)\right]_{\{H=0\}}$ da órbita da restrição algébrica $a=\left[F\left(x_{1}, x_{2}\right) d x_{1} \wedge d x_{2}\right]_{\{H=0\}}$ com respeito ao grupo de simetrias da curva $\{H=0\}$ é igual à dimensão de $\frac{\Lambda^{0}\left(\mathbb{R}^{2}\right)}{\langle\nabla H, F\rangle}$.

Demonstração: De fato, pela Proposição 3.18(1), $\left[\Lambda^{2}\left(\mathbb{R}^{2}\right)\right]_{\{H=0\}}=\left[\Lambda^{2, \text { closed }}\left(\mathbb{R}^{2}\right)\right]_{\{H=0\}}$. Pela demonstração do Teorema 5.2(1), $\left[\Lambda^{2 \text {,closed }}\left(\mathbb{R}^{2}\right)\right]_{\{H=0\}}$ é isomoformo ao $\mathbb{R}$-espaço vetorial $\frac{\Lambda^{0}\left(\mathbb{R}^{2}\right)}{\langle\nabla H\rangle}$, onde o isomorfismo de espaços vetoriais $\rho: \frac{\Lambda^{0}\left(\mathbb{R}^{2}\right)}{\langle\nabla H\rangle} \longrightarrow\left[\Lambda^{2}\left(\mathbb{R}^{2}\right)\right]_{\{H=0\}}$ é dado por $\rho(\bar{f})=\left[f\left(x_{1}, x_{2}\right) d x_{1} \wedge d x_{2}\right]_{\{H=0\}}$. Mais ainda, $\rho$ é um isomorfismo de $\Lambda^{0}\left(\mathbb{R}^{2}\right)$-módulos.

Como $\rho\left(\frac{\langle\nabla H, F\rangle}{\langle H\rangle}\right)=\langle a\rangle$, temos que o $\mathbb{R}$-espaço vetorial $\frac{\Lambda^{0}\left(\mathbb{R}^{2}\right)}{\langle\nabla H, F\rangle}$ é isomorfo ao $\mathbb{R}$-espaço vetorial $\frac{\left[\Lambda^{2}\left(\mathbb{R}^{2}\right)\right]_{\{H=0\}}}{\langle a\rangle}$. Portanto a codimensão de $\langle a\rangle$ é igual a $\operatorname{dim}_{\mathbb{R}} \frac{\Lambda^{0}\left(\mathbb{R}^{2}\right)}{\langle\nabla H, F\rangle}$ e logo pela Proposição 4.23 a codimensão de $a$ em $\left[\Lambda^{2}\left(\mathbb{R}^{2}\right)\right]_{\{H=0\}}$ é $\operatorname{dim}_{\mathbb{R}} \frac{\Lambda^{0}\left(\mathbb{R}^{2}\right)}{\langle\nabla H, F\rangle}$.

O seguinte teorema dá uma forma simples para o cálculo da multiplicidade simplética de qualquer curva quase-homogênea.

Teorema 5.7: Seja $N=\left\{H\left(x_{1}, x_{2}\right)=x_{\geqslant 3}=0\right\}$ uma curva plana quase-homogênea singular no espaço simplético $\left(\mathbb{R}^{2 n}, \omega\right)$. A multiplicidade simplética de $N$ é igual à dimensão de $\frac{\Lambda^{0}\left(\mathbb{R}^{2}\right)}{\left\langle\nabla H, F_{\omega}\right\rangle}$.

Exemplo 5.8: Considere a curva

$$
C=\left\{p_{1}^{2} p_{2}-p_{2}^{3}=0, q_{1}=p_{2}^{3}, q_{2}=0\right\} \subset\left(\mathbb{R}^{4}, \omega_{0}=d p_{1} \wedge d q_{1}+d p_{2} \wedge d q_{2}\right) .
$$

Esta é uma curva plana quase-homogênea difeomorfa à curva

$$
D_{4}=\left\{H\left(x_{1}, x_{2}\right)=x_{1}^{2} x_{2}-x_{2}^{3}=x_{\geqslant 3}=0\right\},
$$

nas coordenadas locais: $x_{1}=p_{1}, x_{2}=p_{2}, x_{3}=q_{1}-p_{2}^{3}, x_{4}=q_{2}, C$ assume a forma $H\left(x_{1}, x_{2}\right)=x_{1}^{2} x_{2}-x_{2}^{3}=x_{\geqslant 3}=0$ e nas mesmas coordenadas a forma $\omega_{0}$ tem a forma $d x_{1} \wedge\left(d x_{3}+3 x_{2}^{2} d x_{2}\right)+d x_{2} \wedge d x_{4}$.

A restrição desta forma à superfície $x_{3}=x_{4}=0$ é $3 x_{2}^{2} d x_{1} \wedge d x_{2}$ e o ideal $\left\langle\nabla H, 3 x_{2}^{2}\right\rangle=$ $\left\langle x_{1}^{2}, x_{1} x_{2}, x_{2}^{2}\right\rangle$.

Pelo Teorema 5.4 o índice de isotropía de $C$ é 2 , pois $3 x_{2}^{2} \in\langle\nabla H\rangle+\mathscr{M}^{2}$ e $3 x_{2}^{2} \notin$ $\langle\nabla H\rangle+\mathscr{M}^{3}$.

Pelo Teorema 5.7 a multiplicidade simplética de $C$ é $3=\operatorname{dim}_{\mathbb{R}} \frac{\Lambda^{0}\left(\mathbb{R}^{2}\right)}{\left\langle x_{1}^{2}, x_{1} x_{2}, x_{2}^{2}\right\rangle}$. 


\subsection{Classificação simplética A-D-E}

Nesta seção damos solução ao Problema A onde $N=\left\{H\left(x_{1}, x_{2}\right)=x_{\geqslant 3}=0\right\}$ e $H$ é uma das singularidades $A_{k}, D_{k}, E_{6}, E_{7}, E_{8}$ de V. Arnol'd.

O Teorema $\mathrm{A}$ e o Teorema 2.12 reduzem o problema $\mathrm{A}$ à classificação de restrições algébricas no espaço $\left[\Lambda^{2}\left(\mathbb{R}^{2}\right)\right]_{\{H=0\}}$ com respeito ao grupo de simetrias locais da curva $\{H=0\} \subset \mathbb{R}^{2}$.

Esta classificação é dada no seguinte teorema.

\begin{tabular}{|l|l|}
\hline$H\left(x_{1}, x_{2}\right)$ & $F_{i}\left(x_{1}, x_{2}\right), i=0,1, \ldots, \mu$ \\
\hline$A_{k}: x_{1}^{k+1}-x_{2}^{2}$, & $F_{0}=1$, \\
$k \geqslant 1$ & $F_{i}=x_{1}^{i}, i=1, \ldots, k-1$ \\
& $F_{k}=0$ \\
\hline & $F_{0}=1$ \\
$D_{k}: x_{1}^{2} x_{2}-x_{2}^{k-1}$, & $F_{i}=b x_{1}+x_{2}^{i}, i=1, \ldots, k-4$ \\
$k \geqslant 4$ & $F_{k-3}=( \pm 1)^{k} x_{1}+b x_{2}^{k-3}$, \\
& $F_{k-2}=x_{2}^{k-3}, F_{k-1}=x_{2}^{k-2}, F_{k}=0$ \\
\hline$E_{6}: x_{1}^{3}-x_{2}^{4}$ & $F_{0}=1, F_{1}= \pm x_{2}+b x_{1}, F_{2}=b x_{2}^{2}+x_{1}$, \\
& $F_{3}=x_{2}^{2}+b x_{1} x_{2}, F_{4}= \pm x_{1} x_{2}, F_{5}=x_{1} x_{2}^{2}, F_{6}=0$ \\
\hline & $F_{0}=1, F_{1}=x_{2}+b x_{1}, F_{2}= \pm x_{1}+b x_{2}^{2}$, \\
$E_{7}: x_{1}^{3}-x_{1} x_{2}^{3}$ & $F_{3}=x_{2}^{2}+b x_{1} x_{2}, F_{4}= \pm x_{1} x_{2}+b x_{2}^{3}$, \\
& $F_{5}=x_{2}^{3}, F_{6}=x_{2}^{4}, F_{7}=0$ \\
\hline & $F_{0}= \pm 1, F_{1}=x_{2}+b x_{1}, F_{2}=x_{1}+b_{1} x_{2}^{2}+b_{2} x_{2}^{3}$, \\
$E_{8}: x_{1}^{3}-x_{2}^{5}$ & $F_{3}= \pm x_{2}^{2}+b x_{1} x_{2}, F_{4}= \pm x_{1} x_{2}+b x_{2}^{3}$, \\
& $F_{5}=x_{2}^{3}+b x_{1} x_{2}^{2}, F_{6}=x_{1} x_{2}^{2}, F_{7}= \pm x_{1} x_{2}^{3}, F_{8}=0$ \\
\hline
\end{tabular}

Tabela 5.1:

Teorema 5.9: Seja $H=H\left(x_{1}, x_{2}\right)$ como na Tabela 5.1. Seja $\mathscr{F}_{i}=\left[F_{i} d x_{1} \wedge d x_{2}\right]_{\{H=0\}}$, onde as funções $F_{i}$ são dadas na linha de $H$ na tabela.

1. Qualquer restrição algébrica $a \in\left[\Lambda^{2}\left(\mathbb{R}^{2}\right)\right]_{\{H=0\}}$ é difeomorfa a uma das formas normais $\mathscr{F}_{i}, i=0, \ldots, \mu$ onde $\mu$ é a multiplicidade de $H$.

2. As formas normais $\mathscr{F}_{0}, \ldots, \mathscr{F}_{\mu}$ são disjuntas.

3. As formas normais $\mathscr{F}_{i}$ tem codimensão $i$.

4. Os parâmetros $b, b_{1}, b_{2}$ nas formas normais são modais, isto é, as restrições algébricas não são equivalentes para diferentes parâmetros. 


\subsubsection{Demonstração do Teorema 5.9(1)}

Para a prova do Teorema 5.9(1) precisamos dos seguintes lemas.

Lema 5.10: Seja $a=\left[x_{1}^{\alpha_{1}} x_{2}^{\alpha_{2}} d x_{1} \wedge d x_{2}\right]_{\{H=0\}}$ e $c \in \mathbb{R}, c \neq 0$, então ca é difeomorfa $a$ \pm a. Se a curva $\{H=0\}$ admite uma simetria local

$$
\left(x_{1}, x_{2}\right) \rightarrow\left(-x_{1}, x_{2}\right) \quad \text { ou } \quad\left(x_{1}, x_{2}\right) \rightarrow\left(x_{1},-x_{2}\right)
$$

que não muda o sinal do monômio $x_{1}^{\alpha_{1}} x_{2}^{\alpha_{2}}$ ou admite uma simetria local

$$
\left(x_{1}, x_{2}\right) \rightarrow\left(-x_{1},-x_{2}\right)
$$

que muda o sinal do monômio $x_{1}^{\alpha_{1}} x_{2}^{\alpha_{2}}$ então as restrições algébricas \pm a são difeomorfas.

\section{Demonstração:}

- Para provar a primeira afirmação suponha que $c>0$, e considere a simetria local $f\left(x_{1}, x_{2}\right)=\left(t_{0}^{\lambda_{1}} x_{1}, t_{0}^{\lambda_{2}} x_{2}\right)$ da curva $\{H=0\}$, onde $t_{0}>0$ satisfaz $t_{0}^{\lambda_{1}\left(\alpha_{1}+1\right)+\lambda_{2}\left(\alpha_{2}+1\right)}=$ c. Portanto

$$
\begin{aligned}
f^{*} a & =\left[t_{0}^{\lambda_{1} \alpha_{1}} x_{1}^{\alpha_{1}} t_{0}^{\lambda_{2} \alpha_{2}} x_{2}^{\alpha_{2}}\left(t_{0}^{\lambda_{1}+\lambda_{2}}\right) d x_{1} \wedge d x_{2}\right]_{\{H=0\}} \\
& =\left[t_{0}^{\lambda_{1}\left(\alpha_{1}+1\right)+\lambda_{2}\left(\alpha_{2}+1\right)} x_{1}^{\alpha_{1}} x_{2}^{\alpha_{2}} d x_{1} \wedge d x_{2}\right]_{\{H=0\}} \\
& =c a .
\end{aligned}
$$

No caso $c<0$, considere $f\left(x_{1}, x_{2}\right)=\left(t_{1}^{\lambda_{1}} x_{1}, t_{1}^{\lambda_{2}} x_{2}\right)$ onde $t_{1}>0$ satifaz a igualdade $t_{1}^{\lambda_{1}\left(\alpha_{1}+1\right)+\lambda_{2}\left(\alpha_{2}+1\right)}=-c$. Portanto

$$
\begin{aligned}
f^{*}(-a) & =\left[-t_{1}^{\lambda_{1} \alpha_{1}} x_{1}^{\alpha_{1}} t_{1}^{\lambda_{2} \alpha_{2}} x_{2}^{\alpha_{2}}\left(t_{1}^{\lambda_{1}+\lambda_{2}}\right) d x_{1} \wedge d x_{2}\right]_{\{H=0\}} \\
& =\left[-t_{1}^{\lambda_{1}\left(\alpha_{1}+1\right)+\lambda_{2}\left(\alpha_{2}+1\right)} x_{1}^{\alpha_{1}} x_{2}^{\alpha_{2}} d x_{1} \wedge d x_{2}\right]_{\{H=0\}} \\
& =c a .
\end{aligned}
$$

- Para a segunda afirmação suponha que $f\left(x_{1}, x_{2}\right)=\left(-x_{1}, x_{2}\right)$ (os outros casos são similares) é uma simetria local da curva que não muda o sinal do monômio $x_{1}^{\alpha_{1}} x_{2}^{\alpha_{2}}$. Assim

$$
\begin{aligned}
f^{*} a & =\left[(-1)^{\alpha_{1}} x_{1}^{\alpha_{1}} x_{2}^{\alpha_{2}}\left(-d x_{1}\right) \wedge d x_{2}\right]_{\{H=0\}}=-\left[x_{1}^{\alpha_{1}} x_{2}^{\alpha_{2}} d x_{1} \wedge d x_{2}\right]_{\{H=0\}} \\
& =-a .
\end{aligned}
$$


Notação: Seja $\omega=f\left(x_{1}, x_{2}\right) d x_{1} \wedge d x_{2} \in \Lambda^{2}\left(\mathbb{R}^{2}\right)$ denotaremos por $[f]$ a restrição algébrica de $\omega$ a $\{H=0\}$.

Observação 5.11: Lembre que se $g, f, h \in \Lambda^{0}\left(\mathbb{R}^{2}\right)$ são tais que $g=h . f$ então $[g]=h .[f]$.

\begin{tabular}{|c|c|c|c|}
\hline$H\left(x_{1}, x_{2}\right)$ & $\lambda_{1}, \lambda_{2}$ & Base de $\left[\Lambda^{2}\left(\mathbb{R}^{2}\right)\right]\{H=0\}$ & Relações \\
\hline$x_{1}^{k+1}-x_{2}^{2}, k \geqslant 1$ & $2, \mathrm{k}+1$ & {$[1],\left[x_{1}\right], \ldots,\left[x_{1}^{k-1}\right]$} & \\
\hline$x_{1}^{2} x_{2}-x_{2}^{k-1}, k \geqslant 4$ & $k-2,2$ & $\begin{array}{l}{[1],\left[x_{2}\right], \ldots,\left[x_{2}^{l}\right],\left[x_{1}\right],} \\
{\left[x_{2}^{l+1}\right], \ldots,\left[x_{2}^{k-2}\right],} \\
\text { onde } l=\llbracket \frac{k-1}{2} \rrbracket\end{array}$ & $\begin{array}{l}{\left[x_{2}^{j}\right]=x_{2}^{j-i} \cdot\left(b\left[x_{1}\right]+\left[x_{2}^{i}\right]\right)} \\
(b \in \mathbb{R}, j>i) ; \\
{\left[x_{2}^{k-2}\right]=\frac{x_{1}}{k-1}\left[x_{1}\right]}\end{array}$ \\
\hline$x_{1}^{3}-x_{2}^{4}$ & 4,3 & $\begin{array}{l}{[1],\left[x_{2}\right],\left[x_{1}\right],\left[x_{2}^{2}\right],} \\
{\left[x_{1} x_{2}\right],\left[x_{1} x_{2}^{2}\right]}\end{array}$ & {$\left[x_{2}^{3}\right]=3 x_{1} \cdot\left[x_{1}\right]$} \\
\hline$x_{1}^{3}-x_{1} x_{2}^{3}$ & 3,2 & $\begin{array}{l}{[1],\left[x_{2}\right],\left[x_{1}\right],\left[x_{2}^{2}\right],} \\
{\left[x_{1} x_{2}\right],\left[x_{2}^{3}\right],\left[x_{2}^{4}\right]}\end{array}$ & \\
\hline$x_{1}^{3}-x_{2}^{5}$ & 5,3 & $\begin{array}{l}{[1],\left[x_{2}\right],\left[x_{1}\right],\left[x_{2}^{2}\right],} \\
{\left[x_{1} x_{2}\right],\left[x_{2}^{3}\right],\left[x_{1} x_{2}^{2}\right],} \\
{\left[x_{1} x_{2}^{3}\right]}\end{array}$ & \\
\hline
\end{tabular}

Tabela 5.2:

Lema 5.12: Com as hipóteses do Teorema 5.9 temos a seguinte Tabela 5.2.

\section{Demonstração:}

- $H\left(x_{1}, x_{2}\right)=x_{1}^{k+1}-x_{2}^{2}$. Logo $\langle\nabla H\rangle=\left\langle x_{1}^{k}, x_{2}\right\rangle$. Portanto a multiplicidade de $H$ é $k$ e $\left\{1, x_{1}, \ldots, x_{1}^{k-1}\right\}$ é uma base para a álgebra local. Logo pela Proposição 3.18 e o Teorema 5.2 temos que $\left\{[1],\left[x_{1}\right], \ldots,\left[x_{1}^{k-1}\right]\right\}$ é uma base de $\left[\Lambda^{2}\left(\mathbb{R}^{2}\right)\right]_{\{H=0\}}$.

- $H\left(x_{1}, x_{2}\right)=x_{1}^{2} x_{2}-x_{2}^{k-1}$. Logo $\langle\nabla H\rangle=\left\langle x_{1} x_{2}, x_{1}^{2}-(k-1) x_{2}^{k}\right\rangle$. Portanto a multiplicidade de $H$ é $k$ e $\left\{1, x_{1}, x_{2}, x_{2}^{2}, \ldots, x_{2}^{k-2}\right\}$ é uma base para a álgebra local. Logo pela Proposição 3.18 e o Teorema 5.2 temos que $\left\{[1],\left[x_{1}\right],\left[x_{2}\right],\left[x_{2}^{2}\right], \ldots,\left[x_{2}^{k-2}\right]\right\}$ é uma base de $\left[\Lambda^{2}\left(\mathbb{R}^{2}\right)\right]_{\{H=0\}}$. Agora tomando $l=\llbracket \frac{k-1}{2} \rrbracket$ podemos reescrever $\left\{[1],\left[x_{2}\right],\left[x_{2}^{2}\right], \ldots,\left[x_{1}\right],\left[x_{2}^{l}\right],\left[x_{2}^{l+1}\right], \ldots,\left[x_{2}^{k-2}\right]\right\}$.

Por outro lado $\left[x_{1}^{2}-(k-1) x_{2}^{k-2}\right]=\left[x_{1} x_{2}\right]=0$ então $\left[x_{1}^{2}\right]=(k-1)\left[x_{2}^{k-2}\right]$ e $\left[x_{1} x_{2}\right]=$ 0 , portanto $\left[x_{2}^{k-2}\right]=\frac{x_{1}}{k-1} \cdot\left[x_{1}\right]$ e $\left[x_{2}^{j}\right]=x_{2}^{j-i} \cdot\left(b\left[x_{1}\right]+\left[x_{2}^{i}\right]\right),(b \in \mathbb{R}, j \geqslant i+1, i \in \mathbb{N})$.

- $H\left(x_{1}, x_{2}\right)=x_{1}^{3}-x_{2}^{4}$. Logo $\langle\nabla H\rangle=\left\langle x_{1}^{2}, x_{2}^{3}\right\rangle$. Portanto a multiplicidade de $H$ é 6 e $\left\{1, x_{2}, x_{1}, x_{2}^{2}, x_{1} x_{2}, x_{1} x_{2}^{2}\right\}$ é uma base para a álgebra local, isto implica que $\left\{[1],\left[x_{2}\right],\left[x_{1}\right],\left[x_{2}^{2}\right],\left[x_{1} x_{2}\right],\left[x_{1} x_{2}^{2}\right]\right\}$ é uma base de $\left[\Lambda^{2}\left(\mathbb{R}^{2}\right)\right]_{\{H=0\}}$. 
- $H\left(x_{1}, x_{2}\right)=x_{1}^{3}-x_{1} x_{2}^{3}$. Logo $\langle\nabla H\rangle=\left\langle 3 x_{1}^{2}-x_{2}^{3}, x_{1} x_{2}^{2}\right\rangle$. Portanto a multiplicidade de $H$ é 7 e $\left\{1, x_{2}, x_{1}, x_{2}^{2}, x_{1} x_{2}, x_{2}^{3}, x_{2}^{4}\right\}$ é uma base para a álgebra local. Assim $\left\{[1],\left[x_{2}\right],\left[x_{1}\right],\left[x_{2}^{2}\right],\left[x_{1} x_{2}\right],\left[x_{2}^{3}\right],\left[x_{2}^{4}\right]\right\}$ é uma base de $\left[\Lambda^{2}\left(\mathbb{R}^{2}\right)\right]_{\{H=0\}}$.

Por outro lado $\left[3 x_{1}^{2}-x_{2}^{3}\right]=0$ então $\left[3 x_{1}^{2}\right]=\left[x_{2}^{3}\right]$, portanto $\left[x_{2}^{3}\right]=3 x_{1} \cdot\left[x_{1}\right]$.

- $H\left(x_{1}, x_{2}\right)=x_{1}^{3}-x_{2}^{5}$. Logo $\langle\nabla H\rangle=\left\langle x_{1}^{2}, x_{2}^{4}\right\rangle$. Portanto a multiplicidade de $H$ é 8 e $\left\{1, x_{2}, x_{1}, x_{2}^{2}, x_{1} x_{2}, x_{2}^{3}, x_{1} x_{2}^{2}, x_{1} x_{2}^{3}\right\}$ é uma base para a álgebra local. Assim $\left\{[1],\left[x_{2}\right],\left[x_{1}\right],\left[x_{2}^{2}\right],\left[x_{1} x_{2}\right],\left[x_{2}^{3}\right],\left[x_{1} x_{2}^{2}\right],\left[x_{1} x_{2}^{3}\right]\right\}$ é uma base de $\left[\Lambda^{2}\left(\mathbb{R}^{2}\right)\right]_{\{H=0\}}$.

Notação: Sejam $a, b \in\left[\Lambda^{2}\left(\mathbb{R}^{2}\right)\right]_{\{H=0\}}$, se $a$ e $b$ são restrições algébricas difeomorfas, escrevemos $a \cong b$.

Observação 5.13: Note que as bases da tabela acima são tomadas tais que satisfazem as condições das Proposições 4.24 e 4.25. De fato, pela Definição 4.17:

- Caso $A_{k},[1],\left[x_{1}\right], \ldots,\left[x_{1}^{k-1}\right]$ têm graus $k+3, k+5, k+7, \ldots, k+3+2(k-1)$ respectivamente pois $\lambda_{1}+\lambda_{2}=k+3$.

- Caso $D_{k}$, distinguimos 2 casos:

$k$ ímpar. Isto implica $2 l=k-1, \operatorname{logo}\left\{[1],\left[x_{2}\right],\left[x_{2}^{2}\right], \ldots,\left[x_{2}^{l-1}\right],\left[x_{1}\right],\left[x_{2}^{l}\right], \ldots,\left[x_{2}^{k-2}\right]\right\}$ têm graus $k, k+2, k+4, \ldots, 2 k-3,2 k-2,2 k-1,2 k+1, \ldots, 3 k-4$ respectivamente pois $\lambda_{1}+\lambda_{2}=k$. Logo $\delta_{0}=k, \delta_{1}=k+2, \ldots, \delta_{l-1}=2 k-3, \delta=2 k-2, \delta_{l}=$ $2 k-1, \ldots, \delta_{k-2}=3 k-4$.

$k$ par. Isto implica $2 l=k-2, \operatorname{logo}\left\{[1],\left[x_{2}\right],\left[x_{2}^{2}\right], \ldots,\left[x_{1}\right],\left[x_{2}^{l}\right], \ldots,\left[x_{2}^{k-2}\right]\right\}$ têm graus $k, k+2, k+4, \ldots, 2 k-2,2 k-2,2 k, \ldots, 3 k-4$ respectivamente. Logo $\delta_{0}=k, \delta_{1}=$ $k+2, \ldots, \delta_{l-1}=2 k-3, \delta=2 k-2, \delta_{l}=2 k-2, \ldots, \delta_{k-2}=3 k-4$.

- $\left\{[1],\left[x_{2}\right],\left[x_{1}\right],\left[x_{2}^{2}\right],\left[x_{1} x_{2}\right],\left[x_{1} x_{2}^{2}\right]\right\}$ têm graus $7,10,11,13,14,17$ respectivamente pois $\lambda_{1}+\lambda_{2}=7$.

- $\left\{[1],\left[x_{2}\right],\left[x_{1}\right],\left[x_{2}^{2}\right],\left[x_{1} x_{2}\right],\left[x_{2}^{3}\right],\left[x_{2}^{4}\right]\right\}$ têm graus $5,7,8,9,10,11,13$ respectivamente pois $\lambda_{1}+\lambda_{2}=5$.

- $\left\{[1],\left[x_{2}\right],\left[x_{1}\right],\left[x_{2}^{2}\right],\left[x_{1} x_{2}\right],\left[x_{2}^{3}\right],\left[x_{1} x_{2}^{2}\right],\left[x_{1} x_{2}^{3}\right]\right\}$ têm graus $8,11,13,14,16,17,19,22$ respectivamente pois $\lambda_{1}+\lambda_{2}=8$.

Agora vejamos a demonstração do Teorema 5.9(1).

\section{Demonstração: do Teorema 5.9(1)}


- $A_{k}$ : seja $a \in\left[\Lambda^{2}\left(\mathbb{R}^{2}\right)\right]_{\{H=0\}}$, então pelo Lema 5.12

$$
a=\sum_{i=0}^{k-1} c_{i}\left[x_{1}^{i}\right] .
$$

Consideremos os seguintes casos:

$-c_{0} \neq 0$.

Como $\left[x_{1}^{i}\right]=x_{1}^{i} \cdot[1] \forall i>0$, pela Proposição $4.25 a \cong c_{0}[1]$. Agora como $\left(x_{1}, x_{2}\right) \rightarrow\left(x_{1},-x_{2}\right)$ é uma simetria local de $\{H=0\}$ que não muda o sinal de 1 , pelo Lema $5.10 c_{0}[1] \cong[1]$. Portanto $a \cong[1]$.

$-c_{0}=c_{1}=\ldots=c_{i-1}=0, c_{i} \neq 0,1 \leqslant i \leqslant k-1$.

Como $\left[x_{1}^{j}\right]=x_{1}^{j-i} \cdot\left[x_{1}^{i}\right] \forall j>i$, pela Proposição $4.25 a \cong c_{i}\left[x_{1}^{i}\right]$. Agora como $\left(x_{1}, x_{2}\right) \rightarrow\left(x_{1},-x_{2}\right)$ é uma simetria local de $\{H=0\}$ que não muda o sinal de $x_{1}^{i}$, pelo Lema $5.10 c_{i}\left[x_{1}^{i}\right] \cong\left[x_{1}^{i}\right]$. Portanto $a \cong\left[x_{1}^{i}\right]$.

$-c_{0}=c_{1}=\ldots=c_{k-1}=0$.

Portanto $a=0$.

- $D_{k}$ : seja $a \in\left[\Lambda^{2}\left(\mathbb{R}^{2}\right)\right]_{\{H=0\}}$, então pelo Lema 5.12

$$
a=\sum_{i=0}^{k-2} c_{i}\left[x_{2}^{i}\right]+c\left[x_{1}\right] .
$$

Distinguimos 2 casos:

$k$ ímpar: note que neste caso ainda podemos usar a Proposição 4.25.

$-c_{0} \neq 0$.

Pela Proposição (4.25) $a \cong c_{0}[1]$. Agora como $\left(x_{1}, x_{2}\right) \rightarrow\left(-x_{1}, x_{2}\right)$ é uma simetria local de $\{H=0\}$ que não muda o sinal de 1 , pelo Lema 5.10 $c_{0}[1] \cong[1]$. Portanto $a \cong[1]$.

$-c_{0}=c_{1}=\ldots=c_{i-1}=0, c_{i} \neq 0,1 \leqslant i \leqslant l-1$.

Pela Proposição $4.25 a \cong c_{i}\left[x_{2}^{i}\right]+\tilde{c}\left[x_{1}\right]$. Agora como $\left(x_{1}, x_{2}\right) \rightarrow\left(-x_{1}, x_{2}\right)$ é uma simetria local de $\{H=0\}$ que não muda o sinal de $x_{2}^{i}$, pelo Lema 5.10 $c_{i}\left[x_{2}^{i}\right]+\tilde{c}\left[x_{1}\right] \cong\left[x_{2}^{i}\right]+b\left[x_{1}\right]$. Portanto $a \cong\left[x_{2}^{i}+b x_{1}\right]$.

$-c_{0}=\ldots=c_{l-1}=0, c \neq 0$

Aqui temos os subcasos

* $c_{l}=\ldots=c_{i-1}=0, c_{i} \neq 0, l \leqslant i \leqslant k-4$.

Usando repetidamente a igualdade $\left[x_{2}^{j}\right]=\frac{x_{2}}{c_{i}}\left(c\left[x_{1}\right]+c_{i}\left[x_{2}^{i}\right]\right), j>i$ obtemos 


$$
\begin{array}{ll}
\left.\begin{array}{l}
j=i+1, \\
j=i+2,
\end{array} \quad c\left[x_{1}\right]+c_{i}\left[x_{2}^{i}\right]+\beta_{i+1} \cong c\left[x_{1}\right]+c_{i}\left[x_{2}^{i}\right]+\beta_{i+2}\right]+c_{i}\left[x_{2}^{i}\right]+\beta_{i+1} & , \beta_{i+1} \in \circ\left(\delta_{i+1}\right) \\
\vdots &
\end{array}
$$$$
\text { muda o sinal de } x_{2}^{i}, c\left[x_{1}\right]+c_{i}\left[x_{2}^{i}\right] \cong\left[x_{2}^{i}\right]+b\left[x_{1}\right] \text {. Portanto } a \cong\left[x_{2}^{i}+b x_{1}\right]
$$$$
* c_{l}=\ldots=c_{k-4}=0, c_{k-3} \neq 0 \text {. }
$$

Pela igualdade $\left[x_{2}^{k-2}\right]=\frac{x_{1}}{k-1}\left[x_{1}\right]$ obtemos $a \cong c\left[x_{1}\right]+c_{k-3}\left[x_{2}^{k-3}\right]$, logo pelo Lema $5.10 c\left[x_{1}\right]+c_{k-3}\left[x_{2}^{k-3}\right] \cong \pm\left[x_{1}\right]+b\left[x_{2}^{k-3}\right]$. Portanto $a \cong\left[ \pm x_{1}+\right.$ $\left.b x_{2}^{k-3}\right]=\left[( \pm)^{k} x_{1}+b x_{2}^{k-3}\right]$

$* c_{l}=\ldots=c_{k-3}=0, c_{k-2} \neq 0$.

Pela igualdade $\left[x_{2}^{k-2}\right]=\frac{x_{1}}{k-1}\left[x_{1}\right] a \cong c\left[x_{1}\right]$, logo pelo Lema 5.10, $c\left[x_{1}\right] \cong$ $\pm\left[x_{1}\right]$. Portanto $a \cong \pm\left[x_{1}\right]$.

$* c_{l}=\ldots=c_{k-2}=0$.

Então $a \cong \pm\left[x_{1}\right]$.

$-c_{0}=\ldots=c_{i-1}=c=0, c_{i} \neq 0, l \leqslant i \leqslant k-2$.

Pela Proposição $4.25 a \cong c_{i}\left[x_{2}^{i}\right]$. Agora como $\left(x_{1}, x_{2}\right) \rightarrow\left(-x_{1}, x_{2}\right)$ é uma simetria local de $\{H=0\}$ que não muda o sinal de $x_{2}^{i}, c_{i}\left[x_{2}^{i}\right] \cong\left[x_{2}^{i}\right]$. Portanto $a \cong\left[x_{2}^{i}\right]$.

$-c_{0}=\ldots=c_{k-2}=c=0$.

Portanto $a=0$.

$k$ par:

$$
-c_{0} \neq 0 \text {. }
$$

Usando as igualdades $\left[x_{2}^{i}\right]=\frac{x_{2}^{i}}{c_{0}} \cdot\left(c_{0}[1]\right), i=1, \ldots, k-2$ e aplicando repetidamente a Proposição 4.24 obtemos 


$$
\begin{array}{rrr}
i=1, & a \cong c_{0}[1]+\beta_{1} & , \beta_{1} \in \circ\left(\delta_{1}\right) \\
i=2, & c_{0}[1]+\beta_{1} \cong c_{0}[1]+\beta_{2} & , \beta_{2} \in \circ\left(\delta_{2}\right) \\
\vdots & & \\
i=l-1, & c_{0}[1]+\beta_{l-2} \cong c_{0}[1]+\beta_{l-1} & , \beta_{l-1} \in \circ\left(\delta_{l-1}\right) \\
{\left[x_{1}\right]=x_{1} \cdot[1],} & c_{0}[1]+\beta_{l-1} \cong c_{0}[1]+\beta & , \beta \in \circ(\delta) \\
i=l, & c_{0}[1]+\beta \cong c_{0}[1]+\beta_{l} & \\
\vdots & & \\
i=k-2, & \left.c_{0}[1]+\beta_{k-3} \cong c_{0}[1]+\beta_{k-2}\right) & , \beta_{k-2} \in \circ\left(\delta_{k-2}\right)
\end{array}
$$

Como $\circ\left(\delta_{k-2}\right)=0$ temos $a \cong c[1]$. Já que $\left(x_{1}, x_{2}\right) \rightarrow\left(-x_{1}, x_{2}\right)$ é uma simetria local de $\{H=0\}$ que não muda o sinal de $1, c_{0}[1] \cong[1]$. Portanto $a \cong[1]$.

$-c_{0}=c_{1}=\ldots=c_{i-1}=0, c_{i} \neq 0,1 \leqslant i \leqslant l-1$.

Usando a igualdade $\left[x_{2}^{j}\right]=x_{2}^{j-i} \cdot\left[x_{2}^{i}\right], j>i$ como no item anterior para $j=$ $i+1, i+2, \ldots, l-1$, temos

$$
a \cong c_{i}\left[x_{i}\right]+\beta_{i+1} \cong c_{i}\left[x_{i}\right]+\beta_{i+2} \cong \ldots \cong c_{i}\left[x_{i}\right]+\beta_{l-1}
$$

De $\beta_{l-1} \in \circ\left(\delta_{l-1}\right)$ temos que existem $\tilde{c}, \tilde{c_{j}} \in \mathbb{R}, j=l, \ldots, k-2$ tais que

$$
\beta_{l-1}=\tilde{c}_{l}\left[x_{2}^{l}\right]+\tilde{c}\left[x_{1}\right]+\sum_{j=l+1}^{k-2} \tilde{c}_{j}\left[x_{2}^{j}\right]
$$

Agora usando $\left[x_{2}^{j}\right]=\frac{x_{2}^{j-i}}{c_{i}}\left(b\left[x_{1}\right]+c_{i}\left[x_{2}^{i}\right]\right), b \in \mathbb{R}, j \geqslant i+1$ para $j=l, l+1, \ldots, k-$ 2 , temos

$c_{i}\left[x_{i}\right]+\beta_{l-1} \cong c_{i}\left[x_{i}\right]+\tilde{c}\left[x_{1}\right]+\beta_{l} \cong c_{i}\left[x_{i}\right]+\tilde{c}\left[x_{1}\right]+\beta_{l+1} \cong \ldots \cong c_{i}\left[x_{2}^{i}\right]+\tilde{c}\left[x_{1}\right]$

Como $\left(x_{1}, x_{2}\right) \rightarrow\left(-x_{1}, x_{2}\right)$ é uma simetria local de $\{H=0\}$ que não muda $o$ sinal de $x_{2}^{i}, c_{i}\left[x_{2}^{i}\right]+\tilde{c}\left[x_{1}\right] \cong\left[x_{2}^{i}\right]+b\left[x_{1}\right]$. Portanto $a \cong\left[x_{2}^{i}+b x_{1}\right]$.

$-c_{0}=\ldots=c_{l-1}=0, c \neq 0$.

Aqui temos os subcasos:

* $c_{l}=\ldots=c_{i-1}=0, c_{i} \neq 0, l \leqslant i \leqslant k-4$.

Usando repetidamente a igualdade $\left[x_{2}^{j}\right]=\frac{x_{2}^{j-i}}{c_{i}}\left(c\left[x_{1}\right]+c_{i}\left[x_{2}^{i}\right]\right), j \geqslant i+1$ para $j=i+1, i+2, \ldots, j=k-2$, obtemos

$$
\begin{aligned}
a & \cong c\left[x_{1}\right]+c_{i}\left[x_{2}^{i}\right]+\beta_{i+1} \cong c\left[x_{1}\right]+c_{i}\left[x_{2}^{i}\right]+\beta_{i+2} \cong \\
& \cong \ldots \cong c\left[x_{1}\right]+c_{i}\left[x_{2}^{i}\right]+\beta_{k-3} \cong c\left[x_{1}\right]+c_{i}\left[x_{2}^{i}\right]
\end{aligned}
$$


Analogamente ao item anterior, $c\left[x_{1}\right]+c_{i}\left[x_{2}^{i}\right] \cong\left[x_{2}^{i}\right]+b\left[x_{1}\right]$. Portanto $a \cong\left[x_{2}^{i}+b x_{1}\right]$.

$* c_{l}=\ldots=c_{k-4}=0, c_{k-3} \neq 0$.

Pela igualdade $\left[x_{2}^{k-2}\right]=\frac{x_{2}}{c_{k-3}}\left(c\left[x_{1}\right]+c_{k-3}\left[x_{2}^{k-3}\right]\right), a \cong c\left[x_{1}\right]+c_{k-3}\left[x_{2}^{k-3}\right]$. Por outro lado, como $k$ é par $\left(x_{1}, x_{2}\right) \rightarrow\left(x_{1},-x_{2}\right)$ é uma simetria local de $\{H=0\}$ que não muda o sinal de $x_{1}$, então $c\left[x_{1}\right]+c_{k-3}\left[x_{2}^{k-3}\right] \cong$ $\left[x_{1}\right]+b\left[x_{2}^{k-3}\right]$. Portanto $a \cong\left[x_{1}+b x_{2}^{k-3}\right]$.

$* c_{l}=\ldots=c_{k-3}=0, c_{k-2} \neq 0$.

Pela igualdade $\left[x_{2}^{k-2}\right]=\frac{x_{1}}{k-1}\left[x_{1}\right], a \cong c\left[x_{1}\right]$ e analogamente ao item anterior temos $c\left[x_{1}\right] \cong\left[x_{1}\right]$. Portanto $a \cong\left[x_{1}\right]$.

$* c_{l}=\ldots=c_{k-2}=0$.

De forma análoga temos $a \cong\left[x_{1}\right]$.

$-c_{0}=\ldots=c_{i-1}=c=0, c_{i} \neq 0, l \leqslant i \leqslant k-2$.

Da igualdade $\left[x_{2}^{j}\right]=\frac{x_{2}^{j-i}}{c_{i}}\left[c_{i} x_{2}^{i}\right], j \geqslant i+1$ obtemos $a \cong c_{i}\left[x_{2}^{i}\right]$. Como $\left(x_{1}, x_{2}\right) \rightarrow$ $\left(-x_{1}, x_{2}\right)$ é uma simetria local de $\{H=0\}$ que não muda o sinal de $x_{2}^{i}$, $c_{i}\left[x_{2}^{i}\right] \cong\left[x_{2}^{i}\right]$. Portanto $a \cong\left[x_{2}^{i}\right]$.

$-c_{0}=\ldots=c_{k-2}=c=0$.

Portanto $a=0$.

Logo obtemos a tabela seguinte

\begin{tabular}{|l|c|}
\hline$c_{0} \neq 0$ & $\mathscr{F}_{0}:[1]$ \\
\hline$c_{0}=\ldots=c_{i-1}=0, c_{i} \neq 0, i \leqslant k-4$ & $\mathscr{F}_{i}:\left[b x_{1}+x_{2}^{i}\right]$ \\
\hline$c_{0}=\ldots=c_{k-4}=0, c \neq 0$ & $\mathscr{F}_{k-3}:\left[( \pm)^{k} x_{1}+b x_{2}^{k-3}\right]$ \\
\hline$c_{0}=\ldots=c_{k-4}=c=0, c_{k-3} \neq 0$ & $\mathscr{F}_{k-2}:\left[x_{2}^{k-3}\right]$ \\
\hline$c_{0}=\ldots=c_{k-3}=c=0, c_{k-2} \neq 0$ & $\mathscr{F}_{k-1}:\left[x_{2}^{k-2}\right]$ \\
\hline$c_{0}=\ldots=c_{k-2}=c=0$ & $\mathscr{F}_{k}:[0]$ \\
\hline
\end{tabular}

Agora já que a prova para os casos $E_{6}, E_{7}$ e $E_{8}$ é similar ao caso $A_{k}$ e $D_{k}$ para $k$ par, somente mencionaremos os fatos que implicam estes resultados.

- $E_{6}$ : seja $a \in\left[\Lambda^{2}\left(\mathbb{R}^{2}\right)\right]_{\{H=0\}}$, então pelo Lema 5.12

$$
a=c_{0}[1]+c_{1}\left[x_{2}\right]+c_{2}\left[x_{1}\right]+c_{3}\left[x_{2}^{2}\right]+c_{4}\left[x_{1} x_{2}\right]+c_{5}\left[x_{1} x_{2}^{2}\right]
$$

Pela Proposição 4.25 e como $\left(x_{1}, x_{2}\right) \rightarrow\left(x_{1},-x_{2}\right)$ é uma simetria local de $\{H=0\}$ que não muda o sinal de $1, x_{1}, x_{2}^{2}, x_{1} x_{2}^{2}$, do Lema 5.10 temos respectivamente que

$$
-c_{0} \neq 0 . \text { Então } a \cong[1]
$$


$-c_{0}=c_{1}=0, c_{2} \neq 0$. Então $a \cong\left[x_{1}+b x_{2}^{2}\right]$.

$-c_{0}=c_{1}=c_{2}=0, c_{3} \neq 0$. Então $a \cong\left[x_{2}^{2}+b x_{1} x_{2}\right]$.

$-c_{0}=c_{1}=c_{2}=c_{3}=c_{4}=0, c_{5} \neq 0$. Então $a \cong\left[x_{1} x_{2}^{2}\right]$.

Agora pela Proposição 4.25 e da primeira parte do Lema 5.10 temos

$-c_{0}=0, c_{1} \neq 0$. Então $a \cong\left[ \pm x_{2}+b x_{1}\right]$.

$-c_{0}=c_{1}=c_{2}=c_{3}=0, c_{4} \neq 0$. Então $a \cong\left[ \pm x_{1} x_{2}\right]$.

$-c_{0}=c_{1}=c_{2}=c_{3}=c_{4}=c_{5}=0$. Então $a=0$.

- $E_{7}$ : seja $a \in\left[\Lambda^{2}\left(\mathbb{R}^{2}\right)\right]_{\{H=0\}}$, então pelo Lema 5.12

$$
a=c_{0}[1]+c_{1}\left[x_{2}\right]+c_{2}\left[x_{1}\right]+c_{3}\left[x_{2}^{2}\right]+c_{4}\left[x_{1} x_{2}\right]+c_{5}\left[x_{2}^{3}\right]+c_{6}\left[x_{2}^{4}\right]
$$

Pela Proposição 4.25 e como $\left(x_{1}, x_{2}\right) \rightarrow\left(-x_{1}, x_{2}\right)$ é uma simetria local de $\{H=0\}$ que não muda o sinal de $1, x_{2}, x_{2}^{2}, x_{2}^{3}, x_{2}^{4}$, do Lema 5.10 temos respectivamente que

$-c_{0} \neq 0$. Então $a \cong[1]$.

$-c_{0}=0, c_{1} \neq 0$. Então $a \cong\left[x_{2}+b x_{1}\right]$.

$-c_{0}=c_{1}=c_{2}=0, c_{3} \neq 0$. Então $a \cong\left[x_{2}^{2}+b x_{1} x_{2}\right]$.

$-c_{0}=c_{1}=c_{2}=c_{3}=c_{4}=0, c_{5} \neq 0$. Então $a \cong\left[x_{2}^{3}\right]$.

$-c_{0}=c_{1}=c_{2}=c_{3}=c_{4}=c_{5}=0, c_{6} \neq 0$. Então $a \cong\left[x_{2}^{3}\right]$.

Agora pela Proposição 4.25 e da primeira parte do Lema 5.10 temos

$-c_{0}=c_{1}=0, c_{2} \neq 0$. Então $a \cong\left[ \pm x_{1}+b x_{2}^{2}\right]$.

$-c_{0}=c_{1}=c_{2}=c_{3}=0, c_{4} \neq 0$. Então $a \cong\left[ \pm x_{1} x_{2}+b x_{2}^{3}\right]$.

$-c_{0}=c_{1}=c_{2}=c_{3}=c_{4}=c_{5}=c_{6}=0$. Então $a=0$.

- $E_{8}$ : seja $a \in\left[\Lambda^{2}\left(\mathbb{R}^{2}\right)\right]_{\{H=0\}}$, então pelo Lema 5.12

$$
a=c_{0}[1]+c_{1}\left[x_{2}\right]+c_{2}\left[x_{1}\right]+c_{3}\left[x_{2}^{2}\right]+c_{4}\left[x_{1} x_{2}\right]+c_{5}\left[x_{2}^{3}\right]+c_{6}\left[x_{1} x_{2}^{2}\right]+c_{7}\left[x_{1} x_{2}^{3}\right]
$$

Pela Proposição 4.25 e como $\left(x_{1}, x_{2}\right) \rightarrow\left(-x_{1},-x_{2}\right)$ é uma simetria local de $\{H=0\}$ que muda o sinal de $x_{2}, x_{1}, x_{2}^{3}, x_{1} x_{2}^{2}$, do Lema 5.10 temos respectivamente que

$-c_{0}=0, c_{1} \neq 0$. Então $a \cong\left[x_{2}+b x_{1}\right]$.

$-c_{0}=c_{1}=0, c_{2} \neq 0$. Então $a \cong\left[x_{1}+b x_{2}^{2}+b_{1} x_{2}^{3}\right]$. 


$$
\begin{aligned}
& -c_{0}=c_{1}=c_{2}=c_{3}=c_{4}=0, c_{5} \neq 0 . \text { Então } a \cong\left[x_{2}^{3}+b x_{1} x_{2}^{2}\right] . \\
& -c_{0}=c_{1}=c_{2}=c_{3}=c_{4}=c_{5}=0, c_{6} \neq 0 . \text { Então } a \cong\left[x_{1} x_{2}^{2}\right] .
\end{aligned}
$$

Agora pela Proposição 4.25 e da primeira parte do Lema 5.10 temos

$$
\begin{aligned}
& -c_{0} \neq 0 . \text { Então } a \cong[ \pm 1] . \\
& -c_{0}=c_{1}=c_{2}=0, c_{3} \neq 0 . \text { Então } a \cong\left[ \pm x_{2}^{2}+b x_{1} x_{2}\right] . \\
& -c_{0}=c_{1}=c_{2}=c_{3}=0, c_{4} \neq 0 . \text { Então } a \cong\left[ \pm x_{1} x_{2}+b x_{2}^{3}\right] . \\
& -c_{0}=c_{1}=c_{2}=c_{3}=c_{4}=c_{5}=c_{6}=0, c_{7} \neq 0 . \text { Então } a \cong \pm\left[x_{1} x_{2}^{3}\right] . \\
& -c_{0}=c_{1}=c_{2}=c_{3}=c_{4}=c_{5}=c_{6}=c_{7}=0 . \text { Então } a=0 .
\end{aligned}
$$

\subsubsection{Demonstração do Teorema 5.9(2)}

Antes de começar a demostração, vamos transferir as formas normais $\mathscr{F}_{i}$ a formas simpléticas seguindo o algoritmo obtido na demonstração da Proposição 2.18.

Fixe qualquer forma simplética, por exemplo $\omega_{0}=d p_{1} \wedge d q_{1}+\cdots+d p_{n} \wedge d q_{n}$.

Se $n \geqslant 2$ então a restrição algébrica $\left[F_{i}\left(x_{1}, x_{2}\right) d x_{1} \wedge d x_{2}\right]_{N}$ pode ser realizada pela forma simplética

$$
\omega_{i}=F_{i} d x_{1} \wedge d x_{2}+d x_{1} \wedge d x_{3}+d x_{2} \wedge d x_{4}+d x_{5} \wedge d x_{6}+\cdots+d x_{2 n-1} \wedge d x_{2 n}
$$

a qual pode ser levada a $\omega_{0}$ pela mudança de coordenadas

$$
\begin{aligned}
& x_{1}=p_{1}, \quad x_{2}=p_{2}, \quad x_{3}=q_{1}-\int_{0}^{p_{2}} F_{i}\left(p_{1}, t\right) d t, \quad x_{4}=q_{2}, \\
& x_{5}=p_{3}, \quad x_{6}=q_{3}, \quad \cdots \quad x_{2 n-1}=p_{n}, \quad x_{2 n}=q_{n} .
\end{aligned}
$$

Esta mudança de coordenadas envia o conjundo em (5.1)

$$
N=\left\{H\left(x_{1}, x_{2}\right)=x_{\geqslant 3}=0\right\}
$$

no conjunto

$$
N^{i}=\left\{H\left(p_{1}, p_{2}\right)=q_{1}-\int_{0}^{p_{2}} F_{i}\left(p_{1}, t\right) d t=q_{\geqslant 2}=p_{\geqslant 3}=0\right\} \subset\left(\mathbb{R}^{2 n}, \omega_{0}\right) .
$$

O anterior mostra que $\left[F_{i}\left(x_{1}, x_{2}\right) d x_{1} \wedge d x_{2}\right]_{N}$ é difeomorfa a $\left[\omega_{0}\right]_{N^{i}}$ para todo $i=$ $0,1, \ldots, \mu$. Então se $\left[F_{i}\left(x_{1}, x_{2}\right) d x_{1} \wedge d x_{2}\right]_{N}$ e $\left[F_{j}\left(x_{1}, x_{2}\right) d x_{1} \wedge d x_{2}\right]_{N}$ são difeomorfas para $i \neq j \operatorname{logo}\left[\omega_{0}\right]_{N^{i}}$ é difeomorfa a $\left[\omega_{0}\right]_{N^{j}}$ e pelo Teorema A (2) $N^{i}$ e $N^{j}$ são simplectomorfos.

Portanto para provar que as formas normais são disjuntas é suficiente distinguir a multiplicidade simplética e o índice de isotropia dos conjuntos dados em (5.2). Assim temos o seguinte lema. 
Lema 5.14: Para os conjuntos $N^{i}$ como em (5.2) o indice de isotropia e a multiplicidade simplética, estão dados na seguinte tabela

\begin{tabular}{|l|l|l|}
\hline $\begin{array}{l}\text { Forma } \\
\text { normal }\end{array}$ & $\begin{array}{l}\text { Ind. de } \\
\text { iso. }\end{array}$ & $\begin{array}{l}\text { Mult. } \\
\text { Sim. }\end{array}$ \\
\hline $\begin{array}{l}A_{k}^{i} \\
0 \leqslant i \leqslant k-1\end{array}$ & $i$ & $i$ \\
\hline$A_{k}^{k}$ & $\infty$ & $k$ \\
\hline \hline$D_{k}^{0}$ & 0 & 0 \\
\hline$D_{k}^{1}$ & 1 & 2 \\
\hline$D_{k}^{i}$ & $b \neq 0: 1$ & $i+1$ \\
$2 \leqslant i \leqslant k-4$ & $b=0: i$ & \\
\hline$D_{k}^{k-3}$ & 1 & $k-2$ \\
\hline$D_{k}^{k-2}$ & $k-3$ & $k-2$ \\
\hline$D_{k}^{k-1}$ & $k-2$ & $k-1$ \\
\hline$D_{k}^{k}$ & $\infty$ & $k$ \\
\hline \hline$E_{6}^{0}$ & 0 & 0 \\
\hline$E_{6}^{1}$ & 1 & 2 \\
\hline$E_{6}^{2}$ & 1 & 3 \\
\hline$E_{6}^{3}$ & 2 & 4 \\
\hline$E_{6}^{4}$ & 2 & 4 \\
\hline$E_{6}^{5}$ & 3 & 5 \\
\hline$E_{6}^{6}$ & $\infty$ & 6 \\
\hline
\end{tabular}

\begin{tabular}{|l|l|l|}
\hline $\begin{array}{c}\text { Forma } \\
\text { normal }\end{array}$ & $\begin{array}{l}\text { Ind. de } \\
\text { iso. }\end{array}$ & $\begin{array}{c}\text { Mult. } \\
\text { Sim. }\end{array}$ \\
\hline$E_{7}^{0}$ & 0 & 0 \\
\hline$E_{7}^{1}$ & 1 & 2 \\
\hline$E_{7}^{2}$ & 1 & 3 \\
\hline$E_{7}^{3}$ & 2 & 4 \\
\hline$E_{7}^{4}$ & 2 & 5 \\
\hline$E_{7}^{5}$ & 3 & 5 \\
\hline$E_{7}^{6}$ & 4 & 6 \\
\hline$E_{7}^{6}$ & $\infty$ & 7 \\
\hline \hline \hline$E_{8}^{0}$ & 0 & 0 \\
\hline$E_{8}^{1}$ & 1 & 2 \\
\hline$E_{8}^{2}$ & 1 & 4 \\
\hline$E_{8}^{3}$ & 2 & 4 \\
\hline$E_{8}^{4}$ & 2 & 5 \\
\hline$E_{8}^{5}$ & 3 & 6 \\
\hline$E_{8}^{6}$ & 3 & 6 \\
\hline$E_{8}^{7}$ & 4 & 7 \\
\hline$E_{8}^{8}$ & $\infty$ & 8 \\
\hline
\end{tabular}

Demonstração: Para provar o Lema usaremos os Teoremas 5.4 e 5.7 em cada caso. Denotaremos por $I$ o índice de isotropía e $m$ a multiplicadade simplética. Em cada caso teremos

$$
\omega=\omega_{i}=F_{i} d x_{1} \wedge d x_{2}+d x_{1} \wedge d x_{3}+d x_{2} \wedge d x_{4}+d x_{5} \wedge d x_{6}+\cdots+d x_{2 n-1} \wedge d x_{2 n}
$$

Portanto $F_{\omega}=F_{i}$.

1. $A_{k}=x_{1}^{k+1}-x_{2}^{2}$ e $\langle\nabla H\rangle=\left\langle x_{1}^{k}, x_{2}\right\rangle$.

- $i=0$.

Assim $F_{\omega}=F_{0}=1$, e pelo Teorema 5.4, $I=0$. Agora como $\left\langle\nabla H, \mathscr{F}_{0}\right\rangle=$ $\Lambda^{0}\left(\mathbb{R}^{2}\right)$, pelo Teorema $5.7 m=0$.

- $1 \leqslant i \leqslant k-1$.

Afirmação: $I=i$

Note que $F_{i} \in\langle\nabla H\rangle+\mathscr{M}^{i}$, logo somente temos que provar $F_{i} \notin\langle\nabla H\rangle+\mathscr{M}^{i+1}$. Suponha que $F_{i} \in\langle\nabla H\rangle+\mathscr{M}^{i+1}$ então existem $f, g, h_{j} \in \Lambda^{0}\left(\mathbb{R}^{2}\right), j=0, \ldots, i+1$ 
tais que

$$
x_{1}^{i}=f\left(x_{1}, x_{2}\right) x_{1}^{k}+g\left(x_{1}, x_{2}\right) x_{2}+\sum_{j=0}^{i+1} h_{j} x_{1}^{j} x_{2}^{i+1-j},
$$

comparando o $i$-jato na origem, temos $x_{1}^{i}=x_{2} j^{i-1}(g)(0)$, que é absurdo. Portanto $F_{i} \notin\langle\nabla H\rangle+\mathscr{M}^{i+1}$ e $I=i$.

Ainda, pelo Teorema 5.7

$$
m=\operatorname{dim} \frac{\Lambda^{0}\left(\mathbb{R}^{2}\right)}{\left\langle\nabla H, F_{\omega}\right\rangle}=\operatorname{dim} \frac{\Lambda^{0}\left(\mathbb{R}^{2}\right)}{\left\langle x_{1}^{k}, x_{2}, x_{1}^{i}\right\rangle}=\operatorname{dim} \frac{\Lambda^{0}\left(\mathbb{R}^{2}\right)}{\left\langle x_{2}, x_{1}^{i}\right\rangle}=i .
$$

- $i=k$.

Assim $F_{\omega}=F_{k}=0 \in\langle\nabla H\rangle$ então pelo Teorema 5.4, I é infinito. Por outro lado, segue do Teorema 5.7 que $m=\operatorname{dim} \frac{\Lambda^{0}\left(\mathbb{R}^{2}\right)}{\left\langle\nabla H, F_{\omega}\right\rangle}=\operatorname{dim} \frac{\Lambda^{0}\left(\mathbb{R}^{2}\right)}{\left\langle x_{1}^{k}, x_{2}\right\rangle}=k$.

2. $D_{k}=x_{1}^{2} x_{2}-x_{2}^{k-1}$ e $\langle\nabla H\rangle=\left\langle x_{1} x_{2}, x_{1}^{2}-(k-1) x_{2}^{k-2}\right\rangle$.

Prosseguindo de modo análogo ao caso $A_{k}$ temos

- $i=0$.

Como $F_{\omega}=F_{0}=1, I=0$ e $m=0$.

- $i=1$.

De $F_{1}=b x_{1}+x_{2}$ segue que $F_{1} \in\langle\nabla H\rangle+\mathscr{M}$ e $F_{1} \notin\langle\nabla H\rangle+\mathscr{M}^{2}$, portanto $I=1$.

Ainda,

$$
m=\operatorname{dim} \frac{\Lambda^{0}\left(\mathbb{R}^{2}\right)}{\left\langle\nabla H, F_{\omega}\right\rangle}=\operatorname{dim} \frac{\Lambda^{0}\left(\mathbb{R}^{2}\right)}{\left\langle x_{1} x_{2}, x_{1}^{2}-(k-1) x_{2}^{k-2}, b x_{1}+x_{2}\right\rangle}=2 .
$$

- $2 \leqslant i \leqslant k-4$.

Temos $F_{i}=b x_{1}+x_{2}^{i}$. Para achar $I$, consideramos os casos.

(a) $b=0$.

Como $F_{i}=x_{2}^{i} \in \mathscr{M}^{i} \subset\langle\nabla H\rangle+\mathscr{M}^{i}$ e $F_{i} \notin\langle\nabla H\rangle+\mathscr{M}^{i+1}$ temos $I=i$.

(b) $b \neq 0$.

Como $F_{i}=b x_{1}+x_{2}^{i} \in \mathscr{M} \subset\langle\nabla H\rangle+\mathscr{M}$ e $F_{i} \notin\langle\nabla H\rangle+\mathscr{M}^{2}$ segue que $I=1$.

Além disso,

$$
m=\operatorname{dim} \frac{\Lambda^{0}\left(\mathbb{R}^{2}\right)}{\left\langle\nabla H, F_{\omega}\right\rangle}=\operatorname{dim} \frac{\Lambda^{0}\left(\mathbb{R}^{2}\right)}{\left\langle x_{1} x_{2}, x_{1}^{2}-(k-1) x_{2}^{k-2}, b x_{1}+x_{2}^{i}\right\rangle}=i+1 .
$$


- $i=k-3$.

De $F_{k-3}=( \pm)^{k} x_{1}+b x_{2}^{k-3} \in \mathscr{M} \subset\langle\nabla H\rangle+\mathscr{M}$ e $F_{k-3} \notin\langle\nabla H\rangle+\mathscr{M}^{2}$ segue que $I=1$.

Temos que

$$
m=\operatorname{dim} \frac{\Lambda^{0}\left(\mathbb{R}^{2}\right)}{\left\langle\nabla H, F_{\omega}\right\rangle}=\operatorname{dim} \frac{\Lambda^{0}\left(\mathbb{R}^{2}\right)}{\left\langle x_{1} x_{2}, x_{1}^{2}-(k-1) x_{2}^{k-2},( \pm)^{k} x_{1}+b x_{2}^{k-3}\right\rangle}=k-2 .
$$

- $i=k-2$.

Temos $F_{k-2}=x_{2}^{k-3} \in \mathscr{M}^{k-3} \subset\langle\nabla H\rangle+\mathscr{M}^{k-3}$ e como $F_{k-2} \notin\langle\nabla H\rangle+\mathscr{M}^{k-2}$ implica $I=k-3$.

Além disso,

$$
m=\operatorname{dim} \frac{\Lambda^{0}\left(\mathbb{R}^{2}\right)}{\left\langle\nabla H, F_{\omega}\right\rangle}=\operatorname{dim} \frac{\Lambda^{0}\left(\mathbb{R}^{2}\right)}{\left\langle x_{1} x_{2}, x_{1}^{2}-(k-1) x_{2}^{k-2}, x_{2}^{k-3}\right\rangle}=k-2 .
$$

- $i=k-1$.

De $F_{k-1}=x_{2}^{k-2} \in \mathscr{M}^{k-2} \subset\langle\nabla H\rangle+\mathscr{M}^{k-2}$ e $F_{k-1} \notin\langle\nabla H\rangle+\mathscr{M}^{k-1}$ segue $I=k-2$.

Ainda,

$$
m=\operatorname{dim} \frac{\Lambda^{0}\left(\mathbb{R}^{2}\right)}{\left\langle\nabla H, F_{\omega}\right\rangle}=\operatorname{dim} \frac{\Lambda^{0}\left(\mathbb{R}^{2}\right)}{\left\langle x_{1} x_{2}, x_{1}^{2}-(k-1) x_{2}^{k-2}, x_{2}^{k-2}\right\rangle}=k-1 .
$$

- $i=k$.

Temos $F_{k-1}=0 \in\langle\nabla H\rangle$ então $I$ é infinito. Além disso

$$
m=\operatorname{dim} \frac{\Lambda^{0}\left(\mathbb{R}^{2}\right)}{\left\langle\nabla H, F_{\omega}\right\rangle}=\operatorname{dim} \frac{\Lambda^{0}\left(\mathbb{R}^{2}\right)}{\left\langle x_{1} x_{2}, x_{1}^{2}-(k-1) x_{2}^{k-2}\right\rangle}=k .
$$

Como nos casos $E_{6}, E_{7}$ e $E_{8}$ os calculos de $I$ e $m$ são análogos aos casos $A_{k}$ e $D_{k}$ somente faremos aqueles que são um pouco mais trabalhosos.

3. $E_{6}=x_{1}^{3}-x_{2}^{4} \quad$ e $\quad\langle\nabla H\rangle=\left\langle x_{1}^{2}, x_{2}^{3}\right\rangle$.

- $i=0, F_{0}=1$.

Então $I=0$ e $m=0$.

- $i=1, F_{1}= \pm x_{2}+b x_{1}$.

Como $F_{1} \in\langle\nabla H\rangle+\mathscr{M}$ e $F_{1} \notin\langle\nabla H\rangle+\mathscr{M}^{2}$ segue $I=1$. Além disso $m=$ $\operatorname{dim} \frac{\Lambda^{0}\left(\mathbb{R}^{2}\right)}{\left\langle\nabla H, F_{\omega}\right\rangle}=\operatorname{dim} \frac{\Lambda^{0}\left(\mathbb{R}^{2}\right)}{\left\langle x_{1}^{2}, x_{2}^{3}, \pm x_{2}+b x_{1}\right\rangle}=2$.

- $i=2, F_{2}=x_{1}+b x_{2}^{2}$.

$F_{2} \in\langle\nabla H\rangle+\mathscr{M}$ e $F_{2} \notin\langle\nabla H\rangle+\mathscr{M}^{2}$ então $I=1$. Além disso $m=$ $\operatorname{dim} \frac{\Lambda^{0}\left(\mathbb{R}^{2}\right)}{\left\langle\nabla H, F_{\omega}\right\rangle}=\operatorname{dim} \frac{\Lambda^{0}\left(\mathbb{R}^{2}\right)}{\left\langle x_{1}^{2}, x_{2}^{3}, x_{1}+b x_{2}^{2}\right\rangle}=3$. 
- $i=3, F_{3}=x_{2}^{2}+b x_{1} x_{2}$.

Temos que $I=2$ pois $F_{3} \in \mathscr{M}^{2} \subset\langle\nabla H\rangle+\mathscr{M}^{2}$ e $F_{3} \notin\langle\nabla H\rangle+\mathscr{M}^{3}$, além disso $m=\operatorname{dim} \frac{\Lambda^{0}\left(\mathbb{R}^{2}\right)}{\left\langle\nabla H, F_{\omega}\right\rangle}=\operatorname{dim} \frac{\Lambda^{0}\left(\mathbb{R}^{2}\right)}{\left\langle x_{1}^{2}, x_{2}^{3}, x_{2}^{2}+b x_{1} x_{2}\right\rangle}=4$.

- $i=4, F_{4}= \pm x_{1} x_{2}$.

$I=2$ pois $F_{4} \in\langle\nabla H\rangle+\mathscr{M}^{2}$ e $F_{4} \notin\langle\nabla H\rangle+\mathscr{M}^{3}$, além disso $m=\operatorname{dim} \frac{\Lambda^{0}\left(\mathbb{R}^{2}\right)}{\left\langle\nabla H, F_{\omega}\right\rangle}=$ $\operatorname{dim} \frac{\Lambda^{0}\left(\mathbb{R}^{2}\right)}{\left\langle x_{1}^{2}, x_{2}^{3}, \pm x_{1} x_{2}\right\rangle}=4$.

- $i=5, F_{5}=x_{1} x_{2}^{2}$.

Temos $F_{5} \in\langle\nabla H\rangle+\mathscr{M}^{3}$. Suponha que $F_{5} \in\langle\nabla H\rangle+\mathscr{M}^{4}$, logo existem $f, g, h_{j} \in \Lambda^{0}\left(\mathbb{R}^{2}\right), j=0, \ldots, 4$ tais que

$$
x_{1} x_{2}^{2}=f x_{1}^{2}+g x_{2}^{3}+\sum_{j=0}^{4} h_{j} x_{1}^{j} x_{2}^{4-j},
$$

comparando o 2-jato na origem, temos $0=f(0) x_{1}^{2}$ isto implica $f(0)=0 \quad$ e pelo Lema de Hadamard $f=x_{1} f_{1}+x_{2} f_{2}$, portanto

$$
x_{1} x_{2}^{2}=f_{1} x_{1}^{3}+f_{2} x_{1}^{2} x_{2}+g x_{2}^{3}+\sum_{j=0}^{4} h_{j} x_{1}^{j} x_{2}^{4-j},
$$

agora comparando o 3-jato na origem

$$
x_{1} x_{2}^{2}=f_{1}(0) x_{1}^{3}+f_{2}(0) x_{1}^{2} x_{2}+g(0) x_{2}^{3}
$$

que é absurdo. Portanto $\mathscr{F}_{5} \notin\langle\nabla H\rangle+\mathscr{M}^{4}$ e $I=3$.

Ainda mais, $m=\operatorname{dim} \frac{\Lambda^{0}\left(\mathbb{R}^{2}\right)}{\left\langle\nabla H, F_{\omega}\right\rangle}=\operatorname{dim} \frac{\Lambda^{0}\left(\mathbb{R}^{2}\right)}{\left\langle x_{1}^{2}, x_{2}^{3}, x_{1} x_{2}^{2}\right\rangle}=5$.

- $i=6, F_{6}=0$.

Então $I$ é infinito e $m=\operatorname{dim} \frac{\Lambda^{0}\left(\mathbb{R}^{2}\right)}{\left\langle\nabla H, F_{\omega}\right\rangle}=\operatorname{dim} \frac{\Lambda^{0}\left(\mathbb{R}^{2}\right)}{\left\langle x_{1}^{2}, x_{2}^{3}\right\rangle}=6$.

4. $E_{7}=x_{1}^{3}-x_{1} x_{2}^{3}$ e $\langle\nabla H\rangle=\left\langle 3 x_{1}^{2}-x_{2}^{3}, x_{1} x_{2}^{2}\right\rangle$.

- $i=0, F_{0}=1$.

Então $I=0$ e $m=0$.

- $i=1, F_{1}=x_{2}+b x_{1}$.

$I=1$, pois $F_{1} \notin\langle\nabla H\rangle+\mathscr{M}^{2}$ e $m=\operatorname{dim} \frac{\Lambda^{0}\left(\mathbb{R}^{2}\right)}{\left\langle\nabla H, F_{\omega}\right\rangle}=\operatorname{dim} \frac{\Lambda^{0}\left(\mathbb{R}^{2}\right)}{\left\langle 3 x_{1}^{2}-x_{2}^{3}, x_{1} x_{2}^{2}, x_{2}+b x_{1}\right\rangle}=2$.

- $i=2, F_{2}= \pm x_{1}+b x_{2}^{2}$.

$I=1$, pois $F_{2} \notin\langle\nabla H\rangle+\mathscr{M}^{2}$ e $m=\operatorname{dim} \frac{\Lambda^{0}\left(\mathbb{R}^{2}\right)}{\left\langle\nabla H, F_{\omega}\right\rangle}=\operatorname{dim} \frac{\Lambda^{0}\left(\mathbb{R}^{2}\right)}{\left\langle 3 x_{1}^{2}-x_{2}^{3}, x_{1} x_{2}^{2}, \pm x_{1}+b x_{2}^{2}\right\rangle}=3$.

- $i=3, F_{3}=x_{2}^{2}+b x_{1} x_{2}$.

$I=2$, pois $F_{3} \notin\langle\nabla H\rangle+\mathscr{M}^{3}$ e $m=\operatorname{dim} \frac{\Lambda^{0}\left(\mathbb{R}^{2}\right)}{\left\langle\nabla H, F_{\omega}\right\rangle}=\operatorname{dim} \frac{\Lambda^{0}\left(\mathbb{R}^{2}\right)}{\left\langle 3 x_{1}^{2}-x_{2}^{3}, x_{1} x_{2}^{2}, x_{2}^{2}+b x_{1} x_{2}\right\rangle}=4$. 
- $i=4, F_{4}= \pm x_{1} x_{2}+b x_{2}^{3}$.

$I=2$ e $m=\operatorname{dim} \frac{\Lambda^{0}\left(\mathbb{R}^{2}\right)}{\left\langle\nabla H, F_{\omega}\right\rangle}=\operatorname{dim} \frac{\Lambda^{0}\left(\mathbb{R}^{2}\right)}{\left\langle 3 x_{1}^{2}-x_{2}^{3}, x_{1} x_{2}^{2}, \pm x_{1} x_{2}+b x_{2}^{3}\right\rangle}=5$.

- $i=5, F_{5}=x_{2}^{3}$.

Temos $F_{5} \in\langle\nabla H\rangle+\mathscr{M}^{3}$ e usando o Lema de Hadamard como no item $E_{6}, i=5$ obtemos $F_{5} \notin\langle\nabla H\rangle+\mathscr{M}^{4}$, então $I=3$.

Além disso, $m=\operatorname{dim} \frac{\Lambda^{0}\left(\mathbb{R}^{2}\right)}{\left\langle\nabla H, F_{\omega}\right\rangle}=\operatorname{dim} \frac{\Lambda^{0}\left(\mathbb{R}^{2}\right)}{\left\langle 3 x_{1}^{2}-x_{2}^{3}, x_{1} x_{2}^{2}, x_{2}^{3}\right\rangle}=5$.

- $i=6, F_{6}=x_{2}^{4}$.

Temos $F_{6} \in \mathscr{M}^{4} \subset\langle\nabla H\rangle+\mathscr{M}^{4}$. Suponha que $F_{6} \in\langle\nabla H\rangle+\mathscr{M}^{5}$, logo existem $f, g, h_{j} \in \Lambda^{0}\left(\mathbb{R}^{2}\right), j=0, \ldots, 5$ tais que

$$
x_{2}^{4}=3 f x_{1}^{2}-f x_{2}^{3}+g x_{1} x_{2}^{2}+\sum_{j=0}^{5} h_{j} x_{1}^{j} x_{2}^{5-j},
$$

comparando o 2-jato na origem, temos $0=3 f(0) x_{1}^{2}$, isto implica $f(0)=0$ e pelo Lema de Hadamard $f=x_{1} f_{1}+x_{2} f_{2}$, portanto

$$
x_{2}^{4}=3 f_{1} x_{1}^{3}+3 f_{2} x_{1}^{2} x_{2}-f_{1} x_{2}^{3} x_{1}-f_{2} x_{2}^{4}+g x_{1} x_{2}^{2}+\sum_{j=0}^{5} h_{j} x_{1}^{j} x_{2}^{5-j},
$$

agora comparando o 3 -jato na origem $0=3 f_{1}(0) x_{1}^{3}+3 f_{2}(0) x_{1}^{2} x_{2}+g(0) x_{1} x_{2}^{2}$ isto implica $f_{1}(0)=f_{2}(0)=g(0)=0$, assim pelo Lema de Hadamard

$$
f_{i}=x_{1} p_{i 1}+x_{2} p_{i 2}, g=x_{1} g_{1}+x_{2} g_{2}, i=1,2
$$

e então

$$
\begin{aligned}
x_{2}^{4}=3 f_{11} x_{1}^{4} & +3 f_{12} x_{1}^{3} x_{2}+3 f_{21} x_{1}^{3} x_{2}+3 f_{22} x_{1}^{2} x_{2}^{2}-f_{11} x_{2}^{3} x_{1}^{2}- \\
& -f_{12} x_{2}^{4} x_{1}-f_{21} x_{2}^{4} x_{1}-f_{22} x_{2}^{5}+g_{1} x_{1}^{2} x_{2}^{2}+g_{1} x_{1} x_{2}^{3}+\sum_{j=0}^{5} h_{j} x_{1}^{j} x_{2}^{5-j} .
\end{aligned}
$$

Comparando o 4-jato temos $x_{2}^{4}=3 f_{11}(0) x_{1}^{4}+\left(3 f_{12}(0)+3 f_{21}(0)\right) x_{1}^{3} x_{2}+3 f_{22}(0) x_{1}^{2} x_{2}^{2}$ que é absurdo, portanto $F_{6} \notin\langle\nabla H\rangle+\mathscr{M}^{5}$ e $I=4$.

Além disso, $m=\operatorname{dim} \frac{\Lambda^{0}\left(\mathbb{R}^{2}\right)}{\left\langle\nabla H, F_{\omega}\right\rangle}=\operatorname{dim} \frac{\Lambda^{0}\left(\mathbb{R}^{2}\right)}{\left\langle 3 x_{1}^{2}-x_{2}^{3}, x_{1} x_{2}^{2}, x_{2}^{4}\right\rangle}=6$.

- $i=7, F_{7}=0$.

Então $I$ é infinito e $m=\operatorname{dim} \frac{\Lambda^{0}\left(\mathbb{R}^{2}\right)}{\left\langle\nabla H, F_{\omega}\right\rangle}=\operatorname{dim} \frac{\Lambda^{0}\left(\mathbb{R}^{2}\right)}{\left\langle 3 x_{1}^{2}-x_{2}^{3}, x_{1} x_{2}^{2}\right\rangle}=7$.

5. $E_{8}=x_{1}^{3}-x_{2}^{5}$ e $\langle\nabla H\rangle=\left\langle x_{1}^{2}, x_{2}^{4}\right\rangle$.

- $i=0, F_{0}= \pm 1$.

Então $I=0$ e $m=0$. 
- $i=1, F_{1}=x_{2}+b x_{1}$.

$I=1$, pois $F_{1} \notin\langle\nabla H\rangle+\mathscr{M}^{2}$ e $m=\operatorname{dim} \frac{\Lambda^{0}\left(\mathbb{R}^{2}\right)}{\left\langle\nabla H, F_{\omega}\right\rangle}=\operatorname{dim} \frac{\Lambda^{0}\left(\mathbb{R}^{2}\right)}{\left\langle x_{1}^{2}, x_{2}^{4}, x_{2}+b x_{1}\right\rangle}=2$.

- $i=2, F_{2}=x_{1}+b_{1} x_{2}^{2}+b_{2} x_{2}^{3}$.

$I=1$, pois $F_{2} \notin\langle\nabla H\rangle+\mathscr{M}^{2}$ e $m=\operatorname{dim} \frac{\Lambda^{0}\left(\mathbb{R}^{2}\right)}{\left\langle\nabla H, F_{\omega}\right\rangle}=\operatorname{dim} \frac{\Lambda^{0}\left(\mathbb{R}^{2}\right)}{\left\langle x_{1}^{2}, x_{2}^{4}, x_{1}+b_{1} x_{2}^{2}+b_{2} x_{2}^{3}\right\rangle}=4$.

- $i=3, F_{3}= \pm x_{2}^{2}+b x_{1} x_{2}$.

$I=2$, pois $F_{3} \notin\langle\nabla H\rangle+\mathscr{M}^{3}$ e $m=\operatorname{dim} \frac{\Lambda^{0}\left(\mathbb{R}^{2}\right)}{\left\langle\nabla H, F_{\omega}\right\rangle}=\operatorname{dim} \frac{\Lambda^{0}\left(\mathbb{R}^{2}\right)}{\left\langle x_{1}^{2}, x_{2}^{4}, \pm x_{2}^{2}+b x_{1} x_{2}\right\rangle}=4$.

- $i=4, F_{4}= \pm x_{1} x_{2}+b x_{2}^{3}$.

$I=2$, pois $F_{4} \notin\langle\nabla H\rangle+\mathscr{M}^{3}$ e $m=\operatorname{dim} \frac{\Lambda^{0}\left(\mathbb{R}^{2}\right)}{\left\langle\nabla H, F_{\omega}\right\rangle}=\operatorname{dim} \frac{\Lambda^{0}\left(\mathbb{R}^{2}\right)}{\left\langle x_{1}^{2}, x_{2}^{4}, \pm x_{1} x_{2}+b x_{2}^{3}\right\rangle}=5$.

- $i=5, F_{5}=x_{2}^{3}+b x_{1} x_{2}^{2}$.

$I=3$, pois $F_{5} \notin\langle\nabla H\rangle+\mathscr{M}^{4}$ e $m=\operatorname{dim} \frac{\Lambda^{0}\left(\mathbb{R}^{2}\right)}{\left\langle\nabla H, F_{\omega}\right\rangle}=\operatorname{dim} \frac{\Lambda^{0}\left(\mathbb{R}^{2}\right)}{\left\langle x_{1}^{2}, x_{2}^{4}, x_{2}^{3}+b x_{1} x_{2}^{2}\right\rangle}=6$.

- $i=6, F_{6}=x_{1} x_{2}^{2}$.

$I=3$ e $m=\operatorname{dim} \frac{\Lambda^{0}\left(\mathbb{R}^{2}\right)}{\left\langle\nabla H, F_{\omega}\right\rangle}=\operatorname{dim} \frac{\Lambda^{0}\left(\mathbb{R}^{2}\right)}{\left\langle x_{1}^{2}, x_{2}^{4}, x_{1} x_{2}^{2}\right\rangle}=6$.

- $i=7, F_{7}= \pm x_{1} x_{2}^{3}$.

$I=4$ e $m=\operatorname{dim} \frac{\Lambda^{0}\left(\mathbb{R}^{2}\right)}{\left\langle\nabla H, F_{\omega}\right\rangle}=\operatorname{dim} \frac{\Lambda^{0}\left(\mathbb{R}^{2}\right)}{\left\langle x_{1}^{2}, x_{2}^{4}, \pm x_{1} x_{2}^{3}\right\rangle}=7$.

- $i=8, F_{8}=0$.

Então $I$ é infinito e $m=\operatorname{dim} \frac{\Lambda^{0}\left(\mathbb{R}^{2}\right)}{\left\langle\nabla H, F_{\omega}\right\rangle}=\operatorname{dim} \frac{\Lambda^{0}\left(\mathbb{R}^{2}\right)}{\left\langle x_{1}^{2}, x_{2}^{4}\right\rangle}=8$.

Agora como a multiplicidades simplética e o índice de isotropia são invariantes simpléticos, temos do lema anterior que as órbitas das formas normais $\mathscr{F}_{i}$ são disjuntas, exceto para os casos

$(\alpha)\left[\left(x_{2}^{2}+b x_{1} x_{2}\right) d x_{1} \wedge d x_{2}\right]_{\{H=0\}}$ e $\left[ \pm x_{1} x_{2} d x_{1} \wedge d x_{2}\right]_{\{H=0\}}, H=x_{1}^{3}-x_{2}^{4}\left(E_{6}\right)$

( $\beta)\left[\left(x_{2}^{3}+b x_{1} x_{2}^{2}\right) d x_{1} \wedge d x_{2}\right]_{\{H=0\}}$ e $\left[x_{1} x_{2}^{2} d x_{1} \wedge d x_{2}\right]_{\{H=0\}}, H=x_{1}^{3}-x_{2}^{5}\left(E_{8}\right)$.

Para distinguir estes casos usaremos o seguinte Lema.

Lema 5.15: Seja $a=\left[F\left(x_{1}, x_{2}\right) d x_{1} \wedge d x_{2}\right]_{N}$, onde $H$ é um polinômio quase-homogêneo. $O$ ideal $\langle\nabla H, F\rangle \subset \Lambda^{0}\left(\mathbb{R}^{2}\right)$ é chamado de ideal associado de a. Considere as restrições $a, \tilde{a} \in\left[\Lambda^{2}\left(\mathbb{R}^{2 n}\right)\right]_{N}$ então se a, a são difeomorfas, os seus ideais associados são isomorfos.

Demonstração: Lembramos que da Proposição 1.49 , dado $f:\left(\mathbb{R}^{2}, 0\right) \rightarrow\left(\mathbb{R}^{2}, 0\right)$ germe de difeomorfismo induz o isomorfismo $f^{*}: \Lambda^{0}\left(\mathbb{R}^{2}\right) \longrightarrow \Lambda^{0}\left(\mathbb{R}^{2}\right)$.

Considere $a=\left[F\left(x_{1}, x_{2}\right) d x_{1} \wedge d x_{2}\right]_{N}$ e $\tilde{a}=\left[\tilde{F}\left(x_{1}, x_{2}\right) d x_{1} \wedge d x_{2}\right]_{N}$ difeomorfas. Seja $M_{0}=\mathbb{R}^{2} \times\{0\}^{2 n-2}$, então o germe de difeomorfismo $\rho:\left(\mathbb{R}^{2}, 0\right) \longrightarrow\left(M_{0}, 0\right)$ dado por 
$\rho\left(x_{1}, x_{2}\right)=\left(x_{1}, x_{2}, 0, \ldots, 0\right)$ torna $\left[F d x_{1} \wedge d x_{2}\right]_{\{H=0\}}$ e $\left[\tilde{F} d x_{1} \wedge d x_{2}\right]_{\{H=0\}}$ difeomorfas, logo existe $\phi$ um germe de difeomorfismo de $\left(\mathbb{R}^{2}, 0\right)$ tal que

$$
\phi^{*}\left(\left[F d x_{1} \wedge d x_{2}\right]_{\{H=0\}}\right)=\left[\tilde{F} d x_{1} \wedge d x_{2}\right]_{\{H=0\}}, \quad \phi(\{H=0\})=\{H=0\}
$$

Provaremos que $\phi^{*}(\langle\nabla H, F\rangle)=\langle\nabla H, \tilde{F}\rangle$, onde $\phi^{*}$ é o isomorfismo induzido. De (5.3) e do Lema 4.4 obtemos

$$
\tilde{F}-F \circ \phi \cdot\left(\frac{\partial \phi_{1}}{\partial x_{1}} \frac{\partial \phi_{2}}{\partial x_{2}}-\frac{\partial \phi_{1}}{\partial x_{2}} \frac{\partial \phi_{2}}{\partial x_{1}}\right) \in\langle\nabla H\rangle
$$

então,

$$
F \circ \phi \cdot\left(\frac{\partial \phi_{1}}{\partial x_{1}} \frac{\partial \phi_{2}}{\partial x_{2}}-\frac{\partial \phi_{1}}{\partial x_{2}} \frac{\partial \phi_{2}}{\partial x_{1}}\right) \in\langle\nabla H, \tilde{F}\rangle
$$

e como $\frac{\partial \phi_{1}}{\partial x_{1}} \frac{\partial \phi_{2}}{\partial x_{2}}-\frac{\partial \phi_{1}}{\partial x_{2}} \frac{\partial \phi_{2}}{\partial x_{1}}$ é invertível

$$
\phi^{*}(F)=F \circ \phi \quad \in\langle\nabla H, \tilde{F}\rangle .
$$

Por outro lado, pela propriedade dos zeros de $H$ temos que existe uma função $f$ tal que

$$
H \circ \phi=H f
$$

derivando com respeito a $x_{i}$, temos

$$
\sum_{j=1}^{2} \frac{\partial H}{\partial x_{j}}(\phi) \cdot \frac{\partial \phi_{j}}{\partial x_{i}}=\frac{\partial H}{\partial x_{i}} f+H \frac{\partial f}{\partial x_{i}}
$$

assim obtemos o sistema

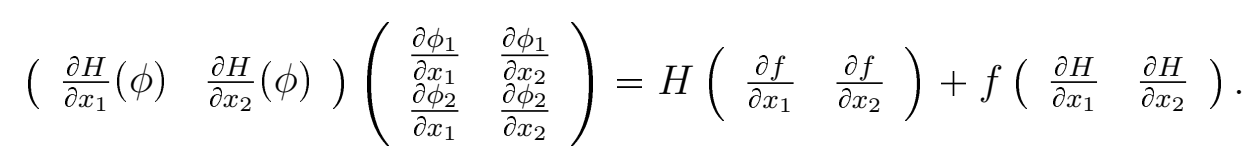

O que implica que

$$
\frac{\partial H}{\partial x_{i}} \circ \phi \in\langle H, \nabla H\rangle=\langle\nabla H\rangle .
$$

Esta última igualdade segue do fato de $H$ ser polinômio quase-homogêneo.

Portanto temos que

$$
\phi^{*}(\langle\nabla H, F\rangle) \subset\langle\nabla H, \tilde{F}\rangle
$$

Agora de (5.3) segue

$$
\left[F d x_{1} \wedge d x_{2}\right]_{\{H=0\}}=\phi^{-1 *}\left(\left[\tilde{F} d x_{1} \wedge d x_{2}\right]_{\{H=0\}}\right), \quad \phi^{-1}(\{H=0\}) \subset\{H=0\}
$$

portanto de forma análoga podemos mostrar

$$
\phi^{*-1}(\langle\nabla H, \tilde{F}\rangle) \subset\langle\nabla H, F\rangle
$$


que implica

$$
\langle\nabla H, \tilde{F}\rangle \subset \phi^{*}(\langle\nabla H, F\rangle) .
$$

$\operatorname{Logo} \phi^{*}(\langle\nabla H, F\rangle)=\langle\nabla H, \tilde{F}\rangle$

Provemos que as classes de $(\alpha)$ e $(\beta)$ não são as mesmas.

Caso $(\alpha)$. Suponha que as restrições são difeomorfas, então existe $\phi=\left(\phi_{1}, \phi_{2}\right)$ germe de difeomorfismo de $\left(\mathbb{R}^{2}, 0\right)$ que leva $\left[ \pm x_{1} x_{2} d x_{1} \wedge d x_{2}\right]_{\{H=0\}}$ em $\left[\left(x_{2}^{2}+b x_{1} x_{2}\right) d x_{1} \wedge d x_{2}\right]_{\{H=0\}}$ e preserva $\{H=0\}$. Pelo Lema de Hadamard aplicado a $\phi_{1}, \phi_{2}$ existem $a_{1}, a_{2}, b_{1}, b_{2} \in$ $\Lambda^{0}\left(\mathbb{R}^{2}\right)$ tais que $\phi_{1}=x_{1} a_{1}+x_{2} a_{2}$ e $\phi_{2}=x_{1} b_{1}+x_{2} b_{2}$.

Por outro lado, pelo Lema $5.15, \phi^{*}$ leva $\left\langle x_{1}^{2}, x_{2}^{3}, x_{1} x_{2}\right\rangle$ em $\left\langle x_{1}^{2}, x_{2}^{3}, x_{2}^{2}+b x_{1} x_{2}\right\rangle$, isto quer dizer

$$
\left\langle x_{1}^{2}(\phi), x_{2}^{3}(\phi), x_{1} x_{2}(\phi)\right\rangle=\left\langle\phi_{1}^{2}, \phi_{2}^{3}, \phi_{1} \phi_{2}\right\rangle=\left\langle x_{1}^{2}, x_{2}^{3}, x_{2}^{2}+b x_{1} x_{2}\right\rangle
$$

logo existem $f_{1}, f_{2}, f_{3}, g_{1}, g_{2}, g_{3} \in \Lambda^{0}\left(\mathbb{R}^{2}\right)$ tais que

$$
\begin{array}{ll}
x_{1}^{2} & =f_{1} \phi_{1}^{2}+f_{2} \phi_{2}^{3}+f_{3} \phi_{1} \phi_{2}, \\
x_{2}^{2}+b x_{1} x_{2} & =g_{1} \phi_{1}^{2}+g_{2} \phi_{2}^{3}+g_{3} \phi_{1} \phi_{2},
\end{array}
$$

comparando os 2-jatos nas equações acima, podemos supor $f_{i}, g_{i}, a_{j}, b_{j} \in \mathbb{R}, i=1,2,3, j=$ 1,2 (somente para evitar a notação $f_{1}(0)$ ) e obtemos

$$
\begin{array}{ll}
x_{1}^{2} & =f_{1}\left(a_{1} x_{1}+a_{2} x_{2}\right)^{2}+f_{3}\left(a_{1} x_{1}+a_{2} x_{2}\right)\left(b_{1} x_{1}+b_{2} x_{2}\right), \\
x_{2}^{2}+b x_{1} x_{2} & =g_{1}\left(a_{1} x_{1}+a_{2} x_{2}\right)^{2}+g_{3}\left(a_{1} x_{1}+a_{2} x_{2}\right)\left(b_{1} x_{1}+b_{2} x_{2}\right),
\end{array}
$$

logo temos o sistema

$$
\left\{\begin{array}{cc}
a_{1}^{2} f_{1}+a_{1} b_{1} f_{3} & =1, \\
2 f_{1} a_{1} a_{2}+f_{3}\left(a_{1} b_{2}+a_{2} b_{1}\right) & =0, \\
a_{2}^{2} f_{1}+f_{3} a_{2} b_{2} & =0, \\
a_{1}^{2} g_{1}+a_{1} b_{1} g_{3} & =0, \\
2 g_{1} a_{1} a_{2}+g_{3}\left(a_{1} b_{2}+a_{2} b_{1}\right) & =b, \\
a_{2}^{2} g_{1}+g_{3} a_{2} b_{2} & =1,
\end{array}\right.
$$

também do fato de $\phi$ ser germe de difeomorfismo temos que $a_{1} b_{2}-b_{1} a_{2} \neq 0$, além disso da primeira e última linha obtemos $a_{1} \neq 0, a_{2} \neq 0, \operatorname{logo} a_{2} f_{1}+f_{3} b_{2}=0$ e portanto

$$
\begin{aligned}
0 & =2 f_{1} a_{1} a_{2}+f_{3}\left(a_{1} b_{2}+a_{2} b_{1}\right) \\
& =-a_{1} f_{3} b_{2}+f_{3} a_{2} b_{1} \\
& =f_{3}\left(a_{2} b_{1}-a_{1} b_{2}\right) .
\end{aligned}
$$

Que implica $f_{3}=0$, logo 


$$
\left\{\begin{array}{l}
a_{1}^{2} f_{1}=1 \\
a_{2} f_{1}=0
\end{array}\right.
$$

que é absurdo, portanto os ideais não são isomorfos e logo as órbitas são disjuntas.

Análogamente para o caso $(\beta)$, supondo que $\phi=\left(\phi_{1}, \phi_{2}\right)$ é germe de difeomorfismo como no item anterior, existem $f_{1}, f_{2}, f_{3}, g_{1}, g_{2}, g_{3} \in \Lambda^{0}\left(\mathbb{R}^{2}\right)$ tais que

$$
\begin{array}{ll}
x_{1}^{2} & =f_{1} \phi_{1}^{2}+f_{2} \phi_{2}^{4}+f_{3} \phi_{1} \phi_{2}^{2}, \\
x_{2}^{3}+b x_{1} x_{2}^{2} & =g_{1} \phi_{1}^{2}+g_{2} \phi_{2}^{4}+g_{3} \phi_{1} \phi_{2}^{2},
\end{array}
$$

comparando os 2-jatos obtemos

$$
\begin{array}{ll}
x_{1}^{2} & =f_{1}(0)\left(a_{1}(0) x_{1}+a_{2}(0) x_{2}\right)^{2}, \\
0 & =g_{1}(0)\left(a_{1}(0) x_{1}+a_{2}(0) x_{2}\right)^{2},
\end{array}
$$

$\operatorname{logo} f_{1}(0), a_{1}(0) \neq 0, g_{1}(0)=a_{2}(0)=0$. Agora comparando os 3 -jatos obtemos

$$
x_{2}^{3}+b x_{1} x_{2}^{2} \quad=j^{1} g_{1}(0)\left(a_{1}(0) x_{1}\right)^{2}+g_{3}(0)\left(a_{1}(0) x_{1}\right)\left(b_{1}(0) x_{1}+b_{2}(0) x_{2}\right)^{2} .
$$

Note que isto é uma contradição, pois na direita não temos o monômio $x_{2}^{3}$ mas na esquerda sim, portanto as órbitas são disjuntas.

\subsubsection{Demonstração do Teorema 5.9(3)}

Seja $a \in\left[\Lambda^{2}\left(\mathbb{R}^{2}\right)\right]_{\{H=0\}}$. Tome uma base $\left[f_{1}\right], \ldots,\left[f_{\mu}\right]$ de $\left[\Lambda^{2}\left(\mathbb{R}^{2}\right)\right]_{\{H=0\}}$ como na Tabela $5.2, \operatorname{logo} a=c_{1}\left[f_{1}\right]+\ldots+c_{\mu}\left[f_{\mu}\right]$ e da prova do Teorema 5.9 (1) podemos verificar o seguinte

- A forma normal $\mathscr{F}_{i}$ que não possui modalidade, tem exatamente os $i$ primeiros coefientes de $c_{1}, c_{2}, \ldots, c_{\mu}$ iguais a zero. Portanto ao calcular o espaço tangente com a fórmula obtemos que a codimensão de $\mathscr{F}_{i}$ é igual a $i$.

- No caso das formas normais que tem modalidade, consideramos a codimensão do estrato, isto é, a codimensão da união das órbitas da família modal. Assím obtemos que esta codimensão é $i$ pois como no caso anterior se $a=c_{1}\left[f_{1}\right]+\ldots+c_{\mu}\left[f_{\mu}\right]$ então $i$ coeficientes de $c_{1}, c_{2}, \ldots, c_{\mu}$ são zero. 


\subsubsection{Demonstração do Teorema 5.9(4)}

Qualquer forma normal com parâmetros no Teorema 5.9 tem a forma $a_{0}+b_{1} a_{1}+\ldots+b_{s} a_{s}$ onde $a_{i}$ são restrições algébricas, $b_{i}$ são parâmetros, $s \leqslant 2$. Para provar que os parâmetros são modais é suficiente mostrar que

$$
a_{i} \notin T\left(a_{0}+b_{1} a_{1}+\ldots+b_{s} a_{s}\right), \forall i
$$

De fato, supondo que a forma normal so tem um parámetro (no outro caso é igual) e supondo que os parâmetros não são modais obtemos que existem $c_{0} \in \mathbb{R}$ e uma vizinhança aberta $I$ de $c_{0}$ tal que $a+t a_{1} \cong a+c_{0} a_{1}$ para todo $t \in I$. Isto significa que existem germes de difeomorfismos $\phi_{t}$ tais que $\phi_{c_{0}}=0$ e $\phi_{t}^{*}\left(a+t a_{1}\right)=a+c_{0} a_{1}$, logo derivando com respeito a $t$ temos $\frac{d \phi_{t}^{*}\left(a+t a_{1}\right)}{d t}=0$ e portanto de (1.3) obtemos $\left.\phi^{*}\left(\mathscr{L}_{X_{t}}\left(a+t a_{1}\right)+a_{1}\right)\right|_{t=c_{0}}=0$ $\left(X_{t}\right.$ é o campo de vetores associado a $\left.\phi_{t}\right)$ que implica $\mathscr{L}_{X_{c_{0}}}\left(a+c_{0} a_{1}\right)=-a_{1}$. Agora de forma análoga à prova da Proposição 4.14 temos que $X_{c_{0}} \in \operatorname{Derlog}\{H=0\}$, então pela Proposição 4.14 obtemos $a_{1} \in T\left(a+c_{0} a_{1}\right)$. Portanto segue que se os parâmetros são modais então $a_{1} \notin T\left(a+c_{0} a_{1}\right)$.

Agora analizemos cada caso:

1. $D_{k}: x_{1}^{2} x_{2}-x_{2}^{k-1}, \quad\left[x_{1} x_{2}\right]=\left[x_{1}^{2}-(k-1) x_{2}^{k-2}\right]=0$.

- $\mathscr{F}_{i}=\left[x_{2}^{i}+b x_{1}\right], 1 \leqslant i \leqslant k-4$.

Obtemos as seguintes relações:

$$
x_{2}^{\alpha_{2}} \mathscr{F}_{i}=\left[x_{2}^{i+\alpha_{2}}\right] ; \quad x_{1}^{\alpha_{1}} \mathscr{F}_{i}=b\left[x_{1}^{1+\alpha_{1}}\right] ; \quad x_{1}^{\alpha_{1}} x_{2}^{\alpha_{2} \mathscr{F}_{i}}=0, \quad \alpha_{1}, \alpha_{2} \geqslant 1 .
$$

Pela Proposição 4.22

$$
T\left(\mathscr{F}_{i}\right)=\mathbb{R}\left\{\sum_{r \in \mathbb{R}} r\left[h \mathscr{F}_{i}\right]^{(r)}, h \in \Lambda^{0}\left(\mathbb{R}^{2}\right)\right\} .
$$

Usando as relações da equação (5.5) obtemos

$$
T\left(\mathscr{F}_{i}\right)=\mathbb{R}\left\{(2 i+k)\left[x_{2}^{i}\right]+(2 k-2) b\left[x_{1}\right], b\left[x_{1}^{2}\right], \quad\left[x_{2}^{i+1}\right], \quad\left[x_{2}^{i+2}\right], \ldots, \quad\left[x_{2}^{k-2}\right]\right\},
$$

e de $\left[x_{2}^{k-2}\right]=\frac{1}{k-1}\left[x_{1}^{2}\right]$, segue

$$
T\left(\mathscr{F}_{i}\right)=\mathbb{R}\left\{(2 i+k)\left[x_{2}^{i}\right]+(2 k-2) b\left[x_{1}\right], \quad\left[x_{1}^{2}\right], \quad\left[x_{2}^{i+1}\right], \quad\left[x_{2}^{i+2}\right], \ldots, \quad\left[x_{2}^{k-3}\right]\right\} .
$$

Suponha $\left[x_{1}\right] \in T\left(\mathscr{F}_{i}\right)$, então

$\left[x_{1}\right]=a\left((2 i+k)\left[x_{2}^{i}\right]+(2 k-2) b\left[x_{1}\right]\right)+d\left[x_{1}^{2}\right]+c_{1}\left[x_{2}^{i+1}\right]+c_{2}\left[x_{2}^{i+2}\right]+\ldots+c_{k-3-i}\left[x_{2}^{k-3}\right]$,

isto implica que $a=d=c_{1}=\ldots=c_{k-3-i}=0 \quad e(2 k-2) a b=1$, que é absurdo. Portanto $\left[x_{1}\right] \notin T\left(\mathscr{F}_{i}\right)$. 
- $\mathscr{F}_{k-3}=\left[( \pm)^{k} x_{1}+b x_{2}^{k-3}\right]$.

Obtemos as relações:

$$
\begin{aligned}
& x_{1}^{\alpha_{1}} x_{2}^{\alpha_{2}} \mathscr{F}_{k-3}=x_{1}^{1+\alpha_{1}} \mathscr{F}_{k-3}=x_{2}^{1+\alpha_{2}} \mathscr{F}_{k-3}=0 ; \frac{x_{1} \mathscr{F}_{k-3}}{( \pm)^{k}(k-1)}=\left[x_{2}^{k-2}\right] ; \\
& x_{2} \mathscr{F}_{i}=\left[b x_{2}^{k-2}\right], \alpha_{1}, \alpha_{2} \geqslant 1 .
\end{aligned}
$$

De forma análoga ao caso anterior, obtemos

$$
T\left(\mathscr{F}_{i}\right)=\mathbb{R}\left\{(2 k-2)( \pm 1)^{k}\left[x_{1}\right]+(3 k-6) b\left[x_{2}^{k-3}\right],( \pm 1)^{k}\left[x_{1}^{2}\right], b\left[x_{2}^{k-2}\right]\right\},
$$

então se $\left[x_{2}^{k-3}\right] \in T\left(\mathscr{F}_{k-3}\right)$, devem existir $c_{1}, c_{2}, c_{3} \in \mathbb{R}$ tais que

$$
\left[x_{2}^{k-3}\right]=c_{1}(2 k-2)( \pm 1)^{k}\left[x_{1}\right]+c_{1}(3 k-6) b\left[x_{2}^{k-3}\right]+c_{2}( \pm 1)^{k}\left[x_{1}^{2}\right]+c_{3} b\left[x_{2}^{k-2}\right],
$$

que implica $c_{1}=c_{2}=c_{3} b=0$ e $c_{1}(3 k-6) b=1$, que é absurdo. Portanto $\left[x_{2}^{k-3}\right] \notin T\left(\mathscr{F}_{k-3}\right)$.

2. $E_{6}: x_{1}^{3}-x_{2}^{4},\left[x_{1}^{2}\right]=\left[x_{2}^{3}\right]=0$.

- $\mathscr{F}_{1}=\left[ \pm x_{2}+b x_{1}\right]$.

Obtemos as relações:

$$
\begin{aligned}
& x_{1} \mathscr{F}_{1}=\left[ \pm x_{1} x_{2}\right] ; x_{2} \mathscr{F}_{1}=\left[ \pm x_{2}^{2}+b x_{1} x_{2}\right] ; x_{1} x_{2} \mathscr{F}_{1}=\left[ \pm x_{1} x_{2}^{2}\right] ; \\
& x_{2}^{2} \mathscr{F}_{1}=b\left[x_{1} x_{2}^{2}\right] ; x_{1}^{\alpha_{1}+1} \mathscr{F}_{1}=x_{2}^{\alpha_{2}+2} \mathscr{F}_{1}=x_{1}^{\alpha_{1}} x_{2}^{\alpha_{2}+1} \mathscr{F}_{1}=0, \alpha_{1}, \alpha_{2} \geqslant 1 .
\end{aligned}
$$

Logo

$$
T\left(\mathscr{F}_{1}\right)=\mathbb{R}\left\{ \pm 10\left[x_{2}\right]+11 b\left[x_{1}\right], \quad\left[x_{1} x_{2}\right], \quad\left[x_{2}^{2}\right], \quad\left[x_{1} x_{2}^{2}\right]\right\},
$$

então se $\left[x_{1}\right] \in T\left(\mathscr{F}_{1}\right)$, devem existir $c_{1}, c_{2}, c_{3}, c_{4} \in \mathbb{R}$ tais que

$$
\left[x_{1}\right]= \pm 10 c_{1}\left[x_{2}\right]+11 b c_{1}\left[x_{1}\right]+c_{2}\left[x_{1} x_{2}\right]+c_{3}\left[x_{2}^{2}\right]+c_{4}\left[x_{1} x_{2}^{2}\right]
$$

que implica $c_{1}=c_{2}=c_{3}=c_{4}=0$ e $11 b c_{1}=1$, que é absurdo. Portanto $\left[x_{1}\right] \notin T\left(\mathscr{F}_{1}\right)$

- $\mathscr{F}_{2}=\left[x_{1}+b x_{2}^{2}\right]$.

Temos as relações:

$$
\begin{aligned}
& x_{1} \mathscr{F}_{2}=b\left[x_{1} x_{2}^{2}\right] ; x_{2} \mathscr{F}_{2}=\left[x_{1} x_{2}\right] ; x_{2}^{2} \mathscr{F}_{2}=\left[x_{1} x_{2}^{2}\right] ; \\
& x_{2}^{\alpha_{2}+2} \mathscr{F}_{2}=x_{1}^{\alpha_{1}+1} \mathscr{F}_{2}=x_{1}^{\alpha_{1}} x_{2}^{\alpha_{2}} \mathscr{F}_{2}=0, \alpha_{1}, \alpha_{2} \geqslant 1 .
\end{aligned}
$$

Logo obtemos

$$
T\left(\mathscr{F}_{2}\right)=\mathbb{R}\left\{11\left[x_{1}\right]+13 b\left[x_{2}^{2}\right], \quad\left[x_{1} x_{2}\right], \quad\left[x_{1} x_{2}^{2}\right]\right\},
$$

portanto $\left[x_{2}^{2}\right] \notin T\left(\mathscr{F}_{2}\right)$. 
- $\mathscr{F}_{3}=\left[x_{2}^{2}+b x_{1} x_{2}\right]$.

Temos as relações:

$$
\begin{aligned}
& x_{1} \mathscr{F}_{3}=\left[x_{1} x_{2}^{2}\right] ; x_{2} \mathscr{F}_{3}=b\left[x_{1} x_{2}^{2}\right] ; \\
& x_{2}^{\alpha_{2}+1} \mathscr{F}_{3}=x_{1}^{\alpha_{1}+1} \mathscr{F}_{3}=x_{1}^{\alpha_{1}} x_{2}^{\alpha_{2}} \mathscr{F}_{3}=0, \alpha_{1}, \alpha_{2} \geqslant 1 .
\end{aligned}
$$

Logo

$$
T\left(\mathscr{F}_{3}\right)=\mathbb{R}\left\{13\left[x_{2}^{2}\right]+14 b\left[x_{1} x_{2}\right],\left[x_{1} x_{2}^{2}\right]\right\},
$$

portanto $\left[x_{1} x_{2}\right] \notin T\left(\mathscr{F}_{3}\right)$.

3. $E_{7}: x_{1}^{3}-x_{1} x_{2}^{3}, \quad 3\left[x_{1}^{2}\right]=\left[x_{2}^{3}\right],\left[x_{1} x_{2}^{2}\right]=0$.

- $\mathscr{F}_{1}=\left[x_{2}+b x_{1}\right]$.

Obtemos

$x_{1} \mathscr{F}_{1}=\left[x_{1} x_{2}+b x_{1}^{2}\right] ; x_{2} \mathscr{F}_{1}=\left[x_{2}^{2}+b x_{1} x_{2}\right] ; x_{1} x_{2} \mathscr{F}_{1}=\left[ \pm x_{1} x_{2}^{2}\right] ;$

$x_{1}^{2} \mathscr{F}_{1}=\left[x_{1}^{2} x_{2}\right]=\frac{1}{3}\left[x_{2}^{4}\right] ; x_{2}^{2} \mathscr{F}_{1}=\left[x_{2}^{3}\right] ; x_{1} x_{2} \mathscr{F}_{1}=b\left[x_{1}^{2} x_{2}\right]=\frac{b}{3}\left[x_{2}^{4}\right] ; x_{2}^{3} \mathscr{F}_{1}=\left[x_{2}^{4}\right] ;$

$x_{1}^{\alpha_{1}+2} \mathscr{F}_{1}=x_{1}^{\alpha_{1}+1} x_{2} \mathscr{F}_{1}=x_{1} x_{2}^{\alpha_{2}+1} \mathscr{F}_{1}=x_{2}^{\alpha_{2}+3} \mathscr{F}_{1}=x_{1}^{\alpha_{1}+1} x_{2}^{\alpha_{2}+1} \mathscr{F}_{1}=0$,

$\alpha_{1}, \alpha_{2} \geqslant 1$.

Logo

$T\left(\mathscr{F}_{1}\right)=\mathbb{R}\left\{7\left[x_{2}\right]+8 b\left[x_{1}\right], 10\left[x_{1} x_{2}\right]+\frac{11}{3}\left[x_{2}^{3}\right], 9\left[x_{2}^{2}\right]+10 b\left[x_{1} x_{2}\right], \quad\left[x_{2}^{3}\right], \quad\left[x_{2}^{4}\right]\right\}$, portanto $\left[x_{1}\right] \notin T\left(\mathscr{F}_{1}\right)$.

- $\mathscr{F}_{2}=\left[ \pm x_{1}+b x_{2}^{2}\right]$.

Temos as relações:

$$
\begin{aligned}
& x_{1} \mathscr{F}_{2}=\left[ \pm x_{1}^{2}\right] ; x_{2} \mathscr{F}_{2}=\left[ \pm x_{1} x_{2}+b x_{2}^{3}\right] ; x_{1} x_{2} \mathscr{F}_{2}=\left[ \pm x_{1}^{2} x_{2}\right]=\frac{1}{3}\left[ \pm x_{2}^{4}\right] ; \\
& x_{1}^{\alpha_{1}+1} \mathscr{F}_{2}=x_{2}^{\alpha_{2}+1} \mathscr{F}_{2}=x_{1}^{\alpha_{1}+1} x_{2}^{\alpha_{2}+1} \mathscr{F}_{2}=0, \alpha_{1}, \alpha_{2} \geqslant 1 .
\end{aligned}
$$

Assim

$$
T\left(\mathscr{F}_{2}\right)=\mathbb{R}\left\{ \pm 8\left[x_{1}\right]+9 b\left[x_{2}^{2}\right], \quad\left[x_{1}^{2}\right], \pm 10\left[x_{1} x_{2}\right]+11\left[x_{2}^{3}\right], \pm\left[x_{2}^{4}\right]\right\},
$$

portanto $\left[x_{2}\right] \notin T\left(\mathscr{F}_{2}\right)$.

- $\mathscr{F}_{3}=\left[x_{2}^{2}+b x_{1} x_{2}\right]$.

Temos as relações:

$$
\begin{aligned}
& x_{1} \mathscr{F}_{3}=b\left[x_{1}^{2} x_{2}\right]=\frac{b}{3}\left[x_{2}^{4}\right] ; x_{2} \mathscr{F}_{3}=\left[x_{2}^{3}\right] ; x_{2}^{2} \mathscr{F}_{3}=\left[x_{2}^{4}\right] ; \\
& x_{1}^{\alpha_{1}+1} \mathscr{F}_{3}=x_{2}^{\alpha_{2}+2} \mathscr{F}_{3}=x_{1}^{\alpha_{1}} x_{2}^{\alpha_{2}} \mathscr{F}_{3}=0, \alpha_{1}, \alpha_{2} \geqslant 1 .
\end{aligned}
$$


Assim

$$
T\left(\mathscr{F}_{3}\right)=\mathbb{R}\left\{9\left[x_{2}^{2}\right]+10 b\left[x_{1} x_{2}\right], \quad\left[x_{2}^{3}\right], \quad\left[x_{2}^{4}\right]\right\},
$$

portanto $\left[x_{3}\right] \notin T\left(\mathscr{F}_{3}\right)$.

- $\mathscr{F}_{4}=\left[ \pm x_{1} x_{2}+b x_{2}^{3}\right]$.

Temos as relações:

$$
\begin{aligned}
& x_{1} \mathscr{F}_{4}=\frac{ \pm 1}{3}\left[x_{2}^{4}\right] ; x_{2} \mathscr{F}_{4}=b\left[x_{4}\right] ; x_{1}^{\alpha_{1}+1} \mathscr{F}_{4}=x_{2}^{\alpha_{2}+1} \mathscr{F}_{4}=x_{1}^{\alpha_{1}} x_{2}^{\alpha_{2}} \mathscr{F}_{4}=0, \\
& \alpha_{1}, \alpha_{2} \geqslant 1
\end{aligned}
$$

Logo

$$
T\left(\mathscr{F}_{4}\right)=\mathbb{R}\left\{ \pm 10\left[x_{1} x_{2}\right]+11 b\left[x_{2}^{3}\right],\left[x_{2}^{4}\right]\right\},
$$

portanto $\left[x_{4}\right] \notin T\left(\mathscr{F}_{4}\right)$.

4. $E_{8}: x_{1}^{3}-x_{2}^{5}, \quad\left[x_{1}^{2}\right]=\left[x_{2}^{4}\right]=0$.

- $\mathscr{F}_{1}=\left[x_{2}+b x_{1}\right]$.

Obtemos as relações:

$$
\begin{aligned}
& x_{1} \mathscr{F}_{1}=\left[x_{1} x_{2}\right] ; x_{2} \mathscr{F}_{1}=\left[x_{2}^{2}+b x_{1} x_{2}\right] ; x_{1} x_{2} \mathscr{F}_{1}=\left[x_{1} x_{2}^{2}\right] ; \\
& x_{2}^{2} \mathscr{F}_{1}=\left[x_{2}^{3}+b x_{1} x_{2}^{2}\right] ; x_{2}^{3} \mathscr{F}_{1}=b\left[x_{1} x_{2}^{3}\right] ; x_{1} x_{2}^{2} \mathscr{F}_{1}=\left[x_{1} x_{2}^{3}\right] ; \\
& x_{1}^{\alpha_{1}+2} \mathscr{F}_{1}=x_{1} x_{2}^{3} \mathscr{F}_{1}=x_{2}^{\alpha_{2}+3} \mathscr{F}_{1}=0, \alpha_{1}, \alpha_{2} \geqslant 1 .
\end{aligned}
$$

$\log 0$

$$
T\left(\mathscr{F}_{1}\right)=\mathbb{R}\left\{11\left[x_{2}\right]+13 b\left[x_{1}\right],\left[x_{1} x_{2}\right],\left[x_{2}^{2}\right],\left[x_{2}^{3}\right], \quad\left[x_{1} x_{2}^{2}\right], \quad\left[x_{1} x_{2}^{3}\right]\right\},
$$

portanto $\left[x_{1}\right] \notin T\left(\mathscr{F}_{1}\right)$.

- $\mathscr{F}_{2}=\left[x_{1}+b_{1} x_{2}^{2}+b_{2} x_{2}^{3}\right]$

Temos as relações:

$x_{1} \mathscr{F}_{2}=\left[b_{1} x_{1} x_{2}+b_{2} x_{1} x_{2}^{3}\right] ; x_{2} \mathscr{F}_{2}=\left[x_{1} x_{2}+b_{1} x_{2}^{3}\right] ; x_{1} x_{2} \mathscr{F}_{1}=b\left[x_{1} x_{2}^{3}\right] ;$

$x_{2}^{2} \mathscr{F}_{2}=\left[x_{1} x_{2}^{2}\right] ; x_{2}^{3} \mathscr{F}_{2}=\left[x_{1} x_{2}^{3}\right] ; x_{1}^{\alpha_{1}+1} x_{2}^{\alpha_{2}-1} \mathscr{F}_{2}=x_{1}^{\alpha_{1}-1} x_{2}^{\alpha_{2}+3} \mathscr{F}_{2}=x_{1} x_{2}^{2} \mathscr{F}_{2}=0$,

$\alpha_{1}, \alpha_{2} \geqslant 1$.

$\log 0$

$T\left(\mathscr{F}_{2}\right)=\mathbb{R}\left\{13\left[x_{1}\right]+14 b_{1}\left[x_{2}^{2}\right]+17 b_{2}\left[x_{2}^{3}\right], 16\left[x_{1} x_{2}\right]+17 b_{1}\left[x_{2}^{3}\right], \quad\left[x_{1} x_{2}^{2}\right], \quad\left[x_{1} x_{2}^{3}\right]\right\}$,

portanto $\left[x_{2}^{2}\right] \notin T\left(\mathscr{F}_{2}\right)$ e $\left[x_{2}^{3}\right] \notin T\left(\mathscr{F}_{2}\right)$. 
- $\mathscr{F}_{3}=\left[ \pm x_{2}^{2}+b x_{1} x_{2}\right]$.

Obtemos as seguintes relações:

$$
\begin{aligned}
& x_{1} \mathscr{F}_{3}=\left[ \pm x_{1} x_{2}^{2}\right] ; x_{2} \mathscr{F}_{3}=\left[ \pm x_{2}^{3}+b x_{1} x_{2}^{2}\right] ; x_{1} x_{2} \mathscr{F}_{3}=\left[ \pm x_{1} x_{2}^{3}\right] ; \\
& x_{2}^{2} \mathscr{F}_{3}=b\left[x_{1} x_{2}^{3}\right] ; x_{1}^{\alpha_{1}+1} \mathscr{F}_{3}=x_{1} x_{2}^{2} \mathscr{F}_{3}=x_{1}^{2} x_{2} \mathscr{F}_{3}=x_{2}^{\alpha_{2}+1} \mathscr{F}_{3}=0, \\
& \alpha_{1}, \alpha_{2} \geqslant 1 .
\end{aligned}
$$

Assim

$$
T\left(\mathscr{F}_{3}\right)=\mathbb{R}\left\{ \pm 14\left[x_{2}^{2}\right]+16 b\left[x_{1} x_{2}\right], \quad\left[x_{1} x_{2}^{2}\right], \pm 14\left[x_{2}^{3}\right]+19\left[x_{1} x_{2}^{2}\right], \quad\left[x_{1} x_{2}^{3}\right]\right\},
$$

portanto $\left[x_{1} x_{2}\right] \notin T\left(\mathscr{F}_{3}\right)$.

- $\mathscr{F}_{4}=\left[ \pm x_{1} x_{2}+b x_{2}^{3}\right]$.

Obtemos as relações:

$$
\begin{aligned}
& x_{1} \mathscr{F}_{4}=b\left[x_{1} x_{2}^{3}\right] ; x_{2} \mathscr{F}_{4}=\left[ \pm x_{1} x_{2}^{2}\right] ; x_{2}^{2} \mathscr{F}_{4}=\left[ \pm x_{1} x_{2}^{3}\right] ; \\
& x_{1}^{\alpha_{1}+1} \mathscr{F}_{4}=x_{1}^{\alpha_{1}} x_{2}^{\alpha_{2}} \mathscr{F}_{4}=x_{2}^{\alpha_{2}+2} \mathscr{F}_{4}=0, \alpha_{1}, \alpha_{2} \geqslant 1
\end{aligned}
$$

Assim

$$
T\left(\mathscr{F}_{4}\right)=\mathbb{R}\left\{ \pm 16\left[x_{1} x_{2}\right]+17 b\left[x_{2}^{3}\right], \quad\left[x_{1} x_{2}^{2}\right], \quad\left[x_{1} x_{2}^{3}\right]\right\},
$$

portanto $\left[x_{2}^{3}\right] \notin T\left(\mathscr{F}_{4}\right)$.

- $\mathscr{F}_{5}=\left[x_{2}^{3}+b x_{1} x_{2}^{2}\right]$.

Temos as relações:

$$
\begin{aligned}
& x_{1} \mathscr{F}_{5}=\left[x_{1} x_{2}^{3}\right] ; x_{2} \mathscr{F}_{5}=b\left[x_{1} x_{2}^{2}\right] ; \\
& x_{1}^{\alpha_{1}+1} \mathscr{F}_{5}=x_{1}^{\alpha_{1}} x_{2}^{\alpha_{2}} \mathscr{F}_{5}=x_{2}^{\alpha_{2}+1} \mathscr{F}_{5}=0, \alpha_{1}, \alpha_{2} \geqslant 1 .
\end{aligned}
$$

Logo

$$
T\left(\mathscr{F}_{5}\right)=\mathbb{R}\left\{17\left[x_{2}^{3}\right]+19 b\left[x_{1} x_{2}^{2}\right], \quad\left[x_{1} x_{2}^{3}\right]\right\},
$$

portanto $\left[x_{1} x_{2}^{2}\right] \notin T\left(\mathscr{F}_{5}\right)$.

Agora já podemos resolver o Problema A, esta resolução será feita em dois teoremas. No primeiro consideramos $n \geqslant 2$ e no segundo fazemos a classificação para $n=1$.

Teorema 5.16: Seja $H=H\left(x_{1}, x_{2}\right)$ como na Tabela (5.1) e considere $n \geqslant 2$. Qualquer curva no espaço simplético $\left(\mathbb{R}^{2 n}, \omega_{0}\right)$, que é difeomorfa à curva $N=\left\{H\left(x_{1}, x_{2}\right)=x_{\geqslant 3}=0\right\}$ pode ser reduzida por um simplectomorfismo a uma e só uma das formas normais $N^{i}$, $i=1,2, \cdots, \mu$ dadas em 5.2, onde $\mu$ é a multiplicadade de $H$. Os parâmetros $b, b_{1}, b_{2}$ são modais simpléticos. A codimensão da classe da singularidade simplética definida pela forma normal $N^{i}$ na classe de todas as curvas difeomorfas a $N$ é igual a $i$. 
Demonstração: Fixe $H$ na tabela (5.1). Seja $\tilde{N}$ uma curva difeomorfa à curva $N$, então existe um germe de difeomorfismo $f:\left(\mathbb{R}^{2 n}, 0\right) \longrightarrow\left(\mathbb{R}^{2 n}, 0\right)$ tal que $f(N, 0)=(\tilde{N}, 0)$. Note que $N \subset \mathbb{R}^{2} \times\{0\}$. Seja $M=f\left(\mathbb{R}^{2} \times\{0\}\right)$, então $(M, 0)$ é germe de subvariedade de mesma dimensão que $\mathbb{R}^{2} \times\{0\}$ e $\tilde{N} \subset M$. Logo

$$
f^{*}\left[\left.\omega_{0}\right|_{T M}\right]_{\tilde{N}}=\left[\left.\left(f^{*} \omega_{0}\right)\right|_{\mathbb{R}^{2} \times\{0\}}\right]_{N} .
$$

Agora pelo Teorema $5.9(2)$ existe $i \in\{1,2, \ldots, \mu\}$ tal que $\left[\left.\left(f^{*} \omega_{0}\right)\right|_{\mathbb{R}^{2} \times\{0\}}\right]_{N}$ é difeomorfa a $\left[\mathscr{F}_{i} d x_{1} \wedge d x_{2}\right]_{N}=\left[\left.\omega_{i}\right|_{\mathbb{R}^{2} \times\{0\}}\right]_{N}$, portanto $\left[\left.\omega_{0}\right|_{T M}\right]_{\tilde{N}}$ é difeomorfa a $\left[\left.\omega_{i}\right|_{\mathbb{R}^{2} \times\{0\}}\right]_{N}$ e pelo Teorema 2.12 temos $\left[\omega_{0}\right]_{\tilde{N}}$ é difeomorfa a $\left[\omega_{i}\right]_{N}$.

Segue da parte inicial da demonstração do Teorema 5.9(2) que $\left[\omega_{i}\right]_{N}$ é difeomorfa a $\left[\omega_{0}\right]_{N^{i}}$, assim pelo Teorema A (2), $N$ é simplectoforma a $N^{i}$.

Teorema 5.17: Seja $H=H\left(x_{1}, x_{2}\right)$ como na tabela (5.1). Todas as curvas no espaço simplético $\left(\mathbb{R}^{2}, \omega_{0}=d p \wedge d q\right)$ que são difeomorfas à curva $\{H=0\}$ são simplectomorfas a não ser que $H=E_{8}$. Qualquer curva em $\left(\mathbb{R}^{2}, d p \wedge d q\right)$ que é difeomorfa a $E_{8}:\left\{x_{1}^{3}-x_{2}^{5}=0\right\}$ é simplectomorfa a um das curvas $p^{3} \pm q^{5}$.

Demonstração: Fixe $H$ na tabela (5.1). Seja $\tilde{N}$ uma curva difeomorfa à curva $N$, então existe um germe de difeomorfismo $f:\left(\mathbb{R}^{2}, 0\right) \longrightarrow\left(\mathbb{R}^{2}, 0\right)$ tal que $f(N, 0)=(\tilde{N}, 0)$. Logo temos que $\left[\omega_{0}\right]_{\tilde{N}}$ e $\left[f^{*} \omega_{0}\right]_{N}$ são difeomorfas, note que $f^{*} \omega_{0}$ é uma forma simplética, pois $f$ é germe de difeomorfismo. Portanto $\left[f^{*} \omega_{0}\right]_{N} \in\left[\operatorname{Symp}\left(\mathbb{R}^{2}\right)\right]_{N}$, logo pelo Teorema $5.2(2)$ $\left[f^{*} \omega_{0}\right]_{N}=\left[\sum_{i=1}^{\mu} c_{i} f_{i} d x_{1} \wedge d x_{2}\right]_{N}$, para alguns $c_{i} \in \mathbb{R}, i=1, \ldots, \mu, c_{1} \neq 0$ e onde $f_{1}=$ $F_{1}, \ldots, f_{\mu}$ é base de $\frac{\Lambda^{0}\left(\mathbb{R}^{2}\right)}{\langle\nabla H\rangle}$. Pela demonstração do Teorema 5.9(2) $\left[\sum_{i=1}^{\mu} c_{i} f_{i} d x_{1} \wedge d x_{2}\right]_{N}$ é difeomorfa a $\left[F_{1} d x_{1} \wedge d x_{2}\right]_{N}$, assim temos que $\left[\omega_{0}\right]_{\tilde{N}}$ é difeomorfa a $\left[F_{1} d x_{1} \wedge d x_{2}\right]_{N}$.

1) Considere o caso $H \neq E_{8}$. Então $F_{1}=1$, logo o germe de difeomorfismo $g\left(x_{1}, x_{2}\right)=$ $(p, q)$ leva $\left[\mathscr{F}_{1} d x_{1} \wedge d x_{2}\right]_{N}$ em $\left[\omega_{0}\right]_{\{H(p, q)=0\}}$, portanto pelo Teorema A (2) $\tilde{N}$ é simplectoforma a $\{H(p, q)=0\}$.

2) No caso $H=E_{8}$ temos que $\mathscr{F}_{1}= \pm 1$. Assim temos $\left[\omega_{0}\right]_{\tilde{N}}$ é difeomorfa a $\left[d x_{1} \wedge d x_{2}\right]_{N}$ ou a $\left[-d x_{1} \wedge d x_{2}\right]_{N}$. No primeiro caso $\tilde{N}$ é simplectomorfa a $\left\{p^{3}-q^{5}=0\right\}$ como no item anterior, no segundo caso, os germes de difeomorfismos $\left(x_{1}, x_{2}\right) \longrightarrow\left(x_{1},-x_{2}\right)$ e $\left(x_{1}, x_{2}\right) \longrightarrow(p, q)$ levam $\left[-d x_{1} \wedge d x_{2}\right]_{N}$ em $[d p \wedge d q]_{\left\{p^{3}+q^{5}=0\right\}}$ e portanto $\tilde{N}$ é simplectomorfo $\mathrm{a}\left\{p^{3}+q^{5}=0\right\}$.

Note que as curvas $\left\{p^{3}-q^{5}=0\right\}$ e $\left\{p^{3}+q^{5}=0\right\}$ não são simplectomorfas, pois as restrições $\left[d x_{1} \wedge d x_{2}\right]_{N}$ e $\left[-d x_{1} \wedge d x_{2}\right]_{N}$ não são difeomorfas. 


\section{Referências Bibliográficas}

[A] V. I. Arnold; First step of local symplectic algebra, Differential topology, infinitedimensional Lie algebras, and applications; D. B. Fuch's 60th anniversary collection, Americam Mathematical Society; Transl. ser. 2, Am. Math. Soc. 194(44) (1999), 1-8.

[A-G] V. I. Arnold, A. B. Givental; Symplectic geometry, in: Dynamical systems, IV. Encyclop. Math. Sci. 4, Springer Berlin (2001) 1-138.

[A-V-G] V. I. Arnold, S. M. Gusein - Zade, A. N. Varchenko; Singularities of Differentiable Maps, vol 1. Birkhä, Boston (1985).

[D-J-Z] W. Domitrz, S. Janeczko, M. Zhitomirskii; Symplectic singularities of varieties: The method of algebraic restrictions; Journal fur die reine und angewandte Mathematik; J. reine angew. Math. 618(2008), 197-235.

[G] C. G. Gibson ; Singular points of smooth mappings. Pitman London-San FranciscoMelbourne (1979).

[I-J] G. Ishikawa, S. Janeczko; Symplectic bifurcations of plane curves and isotropic liftings; Q. J. Math. 54, No. 1 (2003), 73-102.

[K-M] B. Keith, G. Marian ; Differential Geometry and Topology with a View to Dynamical Systems. Chapman \& Hall(2005).

[L] J. M. Lee; Introduction to smooth manifolds. Graduate Texts in Mathematics, No.218. Springer New York. (2013).

[Ly] O. V. Lyashko; Classification of critical points of functions on a manimold with a singular boundary. Funct. Anal. Appl. 17:3 (1983), 187-193.

[M] R. Moussu; Sur l'existence d'intégrales premiéres pour un germe de forme de Pfaff. Ann. Inst. Fourier 26:2 (1976), 171-220. 
[R] H. -J. Reiffen; Das Lemma von Poincaré für holomorphe Differentialformen auf komplexen Räumen, Math. Z., No. 101 (1967), 269-284.

[S] A. C. da Silva; Lectures on Symplectic Geometry. Lecture Notes in Mathematics, Volume 1764. Springer-Verlag Berlin Heidelberg, (2008).

[T] L. W. Tu; An introduction to manifolds. Springer New York. (2011).

[Z] M. Zhitomirskii; Relative Darboux theorem for singular manifolds and local contact algebra, Can. J. Math. 57, No. 6 (2005), 1314-1340. 